Linköping Studies in Science and Technology

Dissertations, No. 1753

\title{
Model-based quantitative assessment of skin microcirculatory blood flow and oxygen saturation
}

\author{
Hanna Jonasson
}

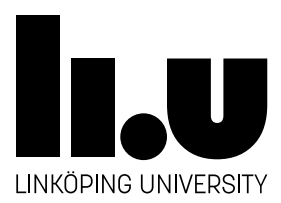

Department of Biomedical Engineering

Linköping University, SE-581 83 Linköping, Sweden

Linköping 2016 
Copyright (C) 2016, Hanna Jonasson. Unless otherwise noted.

Printed in Sweden by LiU-Tryck, Linköping 2016

ISSN 0345-7524

ISBN 978-91-7685-801-1 




\section{Abstract}

The microcirculation, involving the smallest vessels in the body, is where the oxygen transport to all tissue occurs. Evaluating microcirculatory parameters is, therefore, important and involves the quantification of oxygen content of red blood cells (RBCs), the amount of RBCs and their speed.

Diffuse reflectance spectroscopy (DRS) can be used to estimate blood oxygen saturation and fraction of RBCs in tissue since oxygenated and deoxygenated blood have different light absorption characteristics. By illuminating the skin with white light and detecting the spectrum of the backscattered light, tissue absorption and scattering can be assessed. Laser Doppler flowmetry (LDF) is a technique to measure blood flow in tissue. When laser light encounter moving objects in tissue, i.e. RBCs, the light is Doppler shifted, which can be detected and used to calculate tissue perfusion (the fraction of moving RBCs times their speed). With a small distance between light source and detector, both techniques measure superficially where most vessels are microcirculatory vessels. Photon transport in tissue can be simulated with Monte Carlo techniques and the simulations form the basis of modeled DRS and LDF spectra. The estimated microcirculatory parameters are given by the model that best describe measured DRS and LDF data.

This thesis describes the development and the evaluation of an optical method to simultaneously measure oxygen saturation, RBC tissue fraction and speed resolved perfusion in absolute units by integrating DRS and LDF. By combining DRS and LDF into one system with a common tissue model, the two modalities can benefit from each other's strengths. Different calibration methods and model assumptions for the system were evaluated in optical phantoms and in skin measurements. A simple calibration method with two detector distances for DRS was found adequate to accurately estimate absorption and scattering in optical phantoms. It was also necessary to model blood located in vessels, rather than homogeneously distributed in the skin, to obtain accurate parameter estimates. The system was evaluated in healthy subjects during standard provocations, where the parameters were in agreement with other studies and followed an expected pattern during the provocations. In patients with diabetes type 2, tissue fraction of RBCs and nutritive blood flow were reduced in baseline compared to healthy controls. These differences were not related to prevalence of microalbuminuria, a marker sign of microvascular complications in the kidneys.

A combined system with DRS and LDF enables a more comprehensive assessment of the microcirculation by measuring oxygen saturation, RBC tissue fraction and speed resolved perfusion simultaneously and in absolute units. This system has clinical potential to assist in the evaluation of the microcirculation both in healthy and diseased individuals. 



\section{POPULÄRVETENSKAPLIG SAMMANFATTNING}

Mikrocirkulationen innefattar de minsta kärlen i kroppen och det är här syretransporten till all vävnad i kroppen sker. Det är därför viktigt att kunna utvärdera mikrocirkulatoriska parametrar såsom syresättningen hos de röda blodkropparna, mängden röda blodkroppar samt deras hastighet.

Diffus reflektansspektroskopi (DRS) kan användas för att beräkna syresättningen i blodet och mängden röda blodkroppar eftersom syresatt blod har ett karaktäristiskt sätt att absorbera ljus. Absorptionen och spridningen i vävnaden kan skattas genom att belysa huden med vitt ljus och mäta spektrumet från det tillbakaspridda ljuset. Laserdopplerbaserad flödesmätning (LDF) är en teknik som mäter blodflöde i vävnad. När laserljus träffar objekt i vävnaden som rör sig, t.ex. röda blodkroppar, så uppstår Dopplerskift. Dessa Dopplerskift kan detekteras och ett perfusionmått för vävnaden (mängden röda blodkroppar i rörelse gånger deras hastighet) kan beräknas. Med små avstånd mellan ljuskälla och detektor kan båda teknikerna mäta ytligt där den största delen av kärlen tillhör mikrocirkulationen. Fotontransporten i vävnad kan simuleras med Monte Carlo-teknik och simuleringarna ligger till grund för att modellera DRS- och LDF-spektra. De mikrocirkulatoriska parametrarna ges från den modellen som bäst passar DRS- och LDF-data.

Avhandlingen beskriver utvecklingen och utvärderingen av en optisk metod för att simultant mäta syresättningen, mängden röda blodkroppar och hastighetsupplöst perfusion i absoluta enheter genom att integrera DRS och LDF. Genom att kombinera DRS och LDF i ett system med en gemensam hudmodell kan de två modaliteterna dra nytta av varandras styrkor. Olika kalibreringsmetoder och modellantaganden för systemet utvärderades i optiska fantomer och i hudmätningar. En enkel kalibreringsmetod med två detektoravstånd för DRS visade sig vara tillräckligt för att kunna skatta absorption och spridning i optiska fantomer. Det var också nödvändigt att modellera blod i kärl istället för homogent fördelat i huden för att uppnå noggranna parameterskattningar. Systemet utvärderades under standardprovokationer på friska försökspersoner där parametrarna stämde överens med andra studier och följde ett förväntat mönster under provokationerna. Hos patienter med diabetes typ 2 sågs en minskad mängd röda blodkroppar och kapillärt blodflöde i oprovocerad hud jämfört med friska kontroller. Skillnaden var inte kopplad till förekomsten av mikroalbuminuri, ett tecken på mikrovaskulära komplikationer i njurarna.

Ett kombinerat system med DRS och LDF ger en mer fullständig bild av mikrocirkulationen genom att samtidigt och i absoluta enheter mäta syresättningen, mängden röda blodkroppar och hastighetsupplöst perfusion. Systemet kan användas för att utvärdera mikrocirkulationen både hos friska och sjuka individer. 



\section{LIST OF PAPERS}

This thesis is based on the following five papers, referenced in the text with their roman numerals:

I. H. Karlsson, A. Pettersson, M. Larsson, and T. Strömberg, "Can a one-layer optical skin model including melanin and inhomogeneously distributed blood explain spatially resolved diffuse reflectance spectra?", Proceedings of SPIE 7896, 78962Y, 78962Y-9 (2011)

II. H. Karlsson, I. Fredriksson, M. Larsson and T. Strömberg, "Inverse Monte Carlo for estimation of scattering and absorption in liquid optical phantoms", Optics Express 2012233 12246 (2012)

III. T. Strömberg, H. Karlsson, I. Fredriksson, F.H. Nyström and M. Larsson, "Microcirculation assessment using an individualized model for diffuse reflectance spectroscopy and conventional laser Doppler flowmetry”, Journal of Biomedical Optics 19 (5): p. 57002 (2014)

IV. H. Jonasson, I. Fredriksson, A. Pettersson, M. Larsson and T. Strömberg, “Oxygen saturation, red blood cell tissue fraction and speed resolved perfusion - A new optical method for microcirculatory assessment”, Microvascular Research 102 p.70-77 (2015)

V. H. Jonasson, S. Bergstrand, F.H. Nyström, T. Länne, C.J. Östgren, N. Bjarnegård, I. Fredriksson, M. Larsson and T. Strömberg, "Type 2 diabetes is associated with impaired microvascular function in the skin independently of microalbuminuria", Submitted.

The following paper are related to the thesis, but not included:

T. Strömberg, H. Karlsson, I. Fredriksson, M. Larsson, "Experimental results using a threelayer skin model for diffuse reflectance spectroscopy”, Proceedings of SPIE 8578, 857834-1 (2013)

H. Jonasson, I. Fredriksson, M. Larsson, T. Strömberg, "Assessment of the microcirculation using combined model based diffuse reflectance spectroscopy and laser Doppler flowmetry", 16th Nordic-Baltic Conference on Biomedical Engineering, IFMBE Proceedings, Vol. 48, 5254 (2014) 



\section{AbBreviations}

\begin{tabular}{|c|c|}
\hline ACR & Albumin/creatinine ratio \\
\hline $\mathrm{CMBC}$ & Concentration of moving blood cells \\
\hline $\mathrm{CMRO}_{2}$ & Cerebral metabolic rate of oxygen \\
\hline CT & Computed tomography \\
\hline $\mathrm{CV}$ & Coefficient of variation \\
\hline DCS & Diffuse correlation spectroscopy \\
\hline DOS & Diffuse optical spectroscopy \\
\hline DRS & Diffuse reflectance spectroscopy \\
\hline EDHF & Endothelial-derived hyperpolarizing factor \\
\hline EPOS & Enhanced perfusion and oxygen saturation \\
\hline GK & Gegenbauer kernel \\
\hline GPU & Graphics processing unit \\
\hline $\mathrm{Hb}$ & Hemoglobin \\
\hline HbA1c & Glycated hemoglobin \\
\hline $\mathrm{HbO}_{2}$ & Oxygenized hemoglobin \\
\hline HG & Henyey-Greenstein \\
\hline $\mathrm{LDF}$ & Laser Doppler flowmetry \\
\hline $\mathrm{MC}$ & Monte Carlo \\
\hline $\mathrm{MMRO}_{2}$ & Mammary metabolic rate of oxygen \\
\hline MRI & Magnetic resonance imaging \\
\hline NIRS & Near-infrared spectroscopy \\
\hline NO & Nitric oxide \\
\hline OCT & Optical coherence tomography \\
\hline PAD & Peripheral arterial disease \\
\hline PDT & Photodynamic therapy \\
\hline PORH & Post-occlusive reactive hyperemia \\
\hline PU & Perfusion units \\
\hline $\mathrm{RBC}$ & Red blood cell \\
\hline SCAPIS & Swedish CardioPulmonary BioImage Study \\
\hline SCT & Spectral collimated transmission \\
\hline SDF & Side stream darkfield \\
\hline SRDR & Spatially resolved diffuse reflectance \\
\hline $\operatorname{tcpO}_{2}$ & Transcutaneous oxygen pressure \\
\hline UV & Ultraviolet \\
\hline
\end{tabular}





\section{TABLE OF CONTENTS}

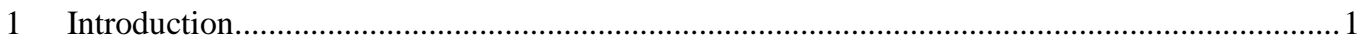

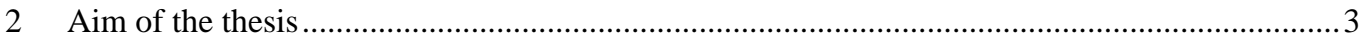

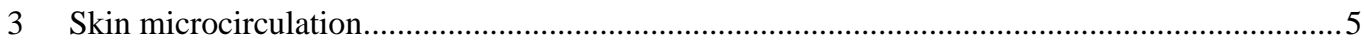

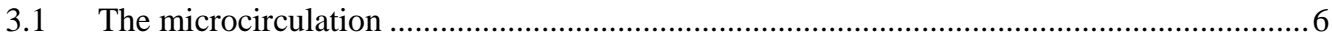

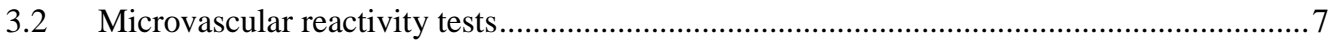

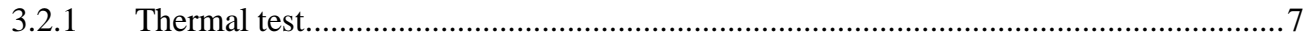

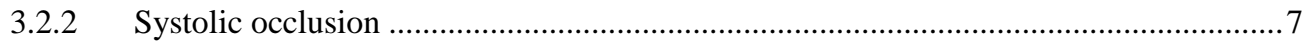

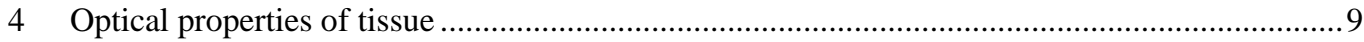

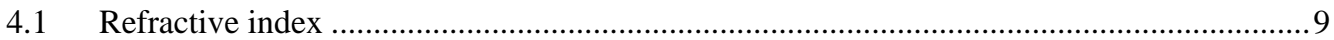

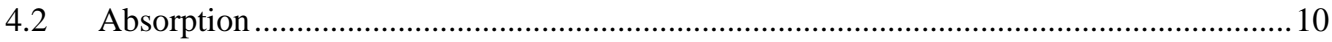

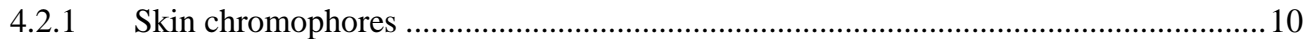

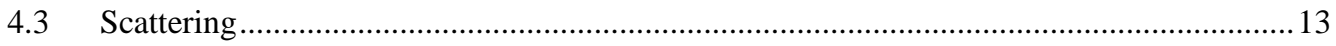

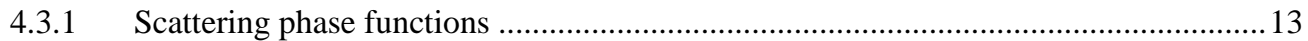

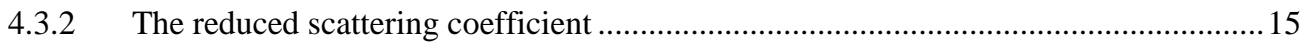

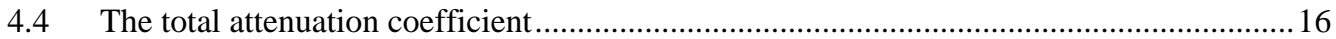

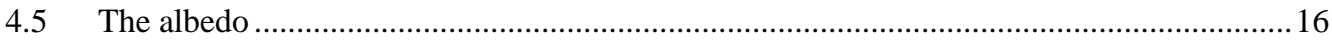

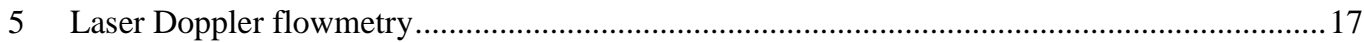

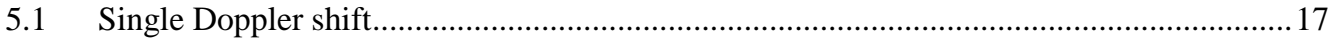

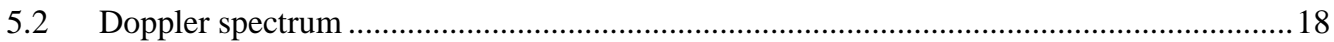

5.3 Concentration of red blood cells and perfusion.......................................................... 19

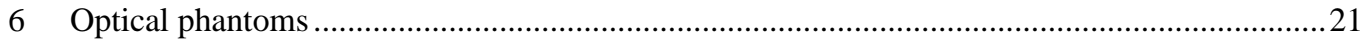

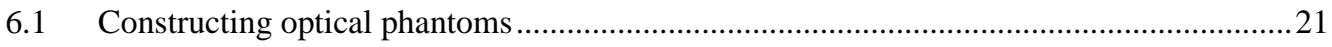

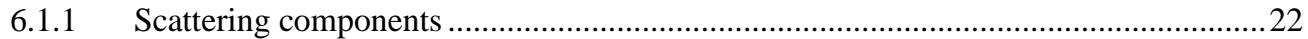

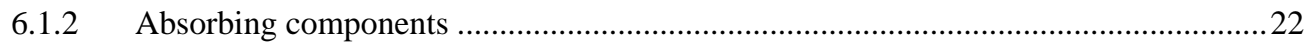

6.2 Measuring optical properties of phantoms ..................................................................2 23

6.2.1 Spectral collimated transmission ...................................................................... 23

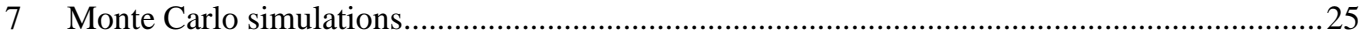




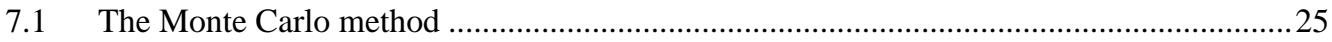

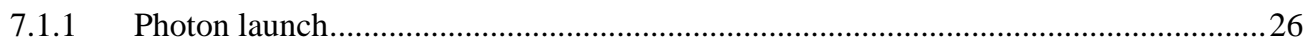

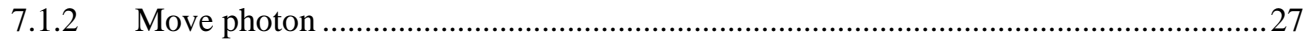

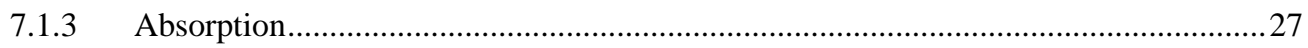

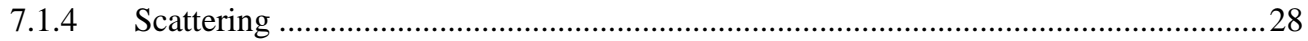

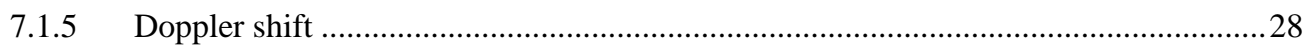

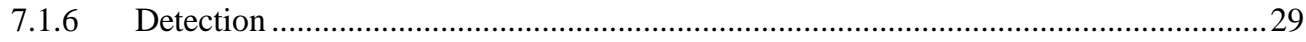

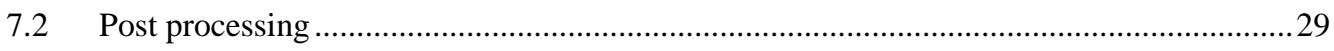

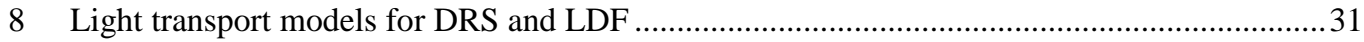

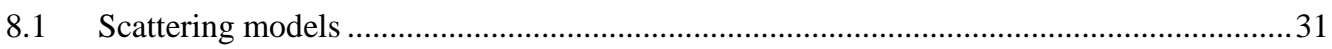

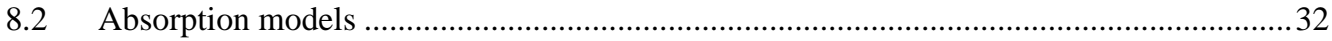

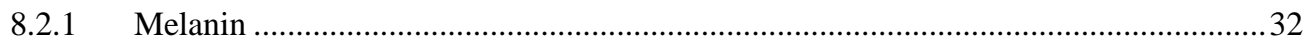

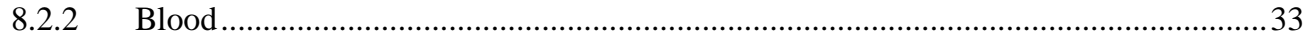

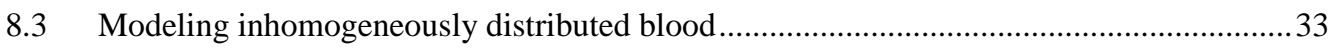

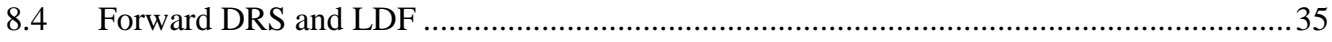

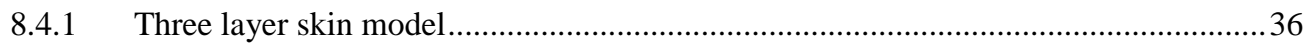

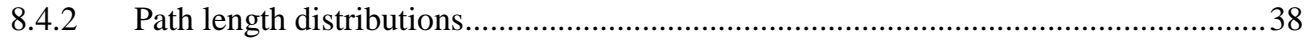

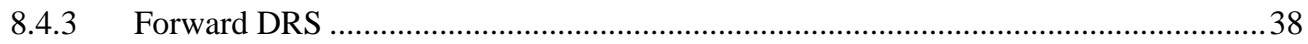

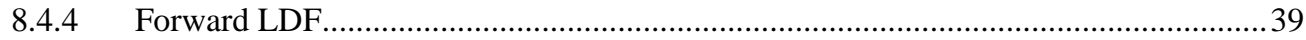

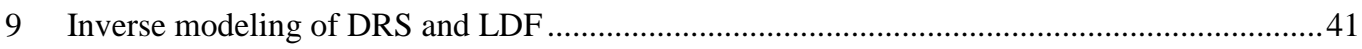

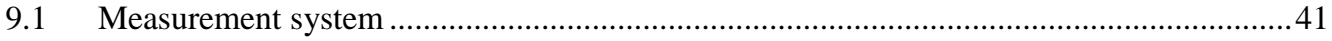

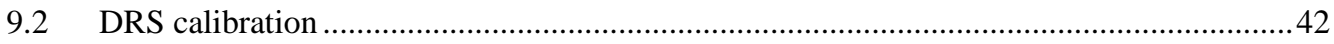

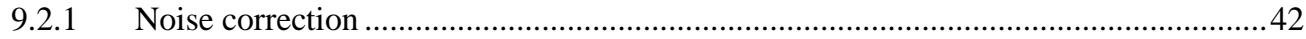

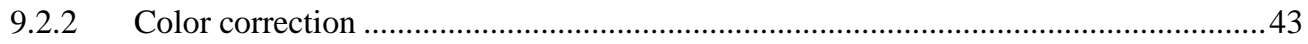

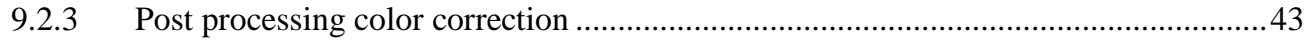

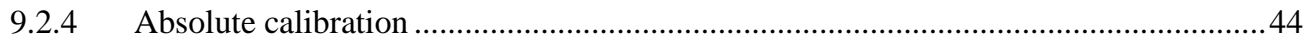

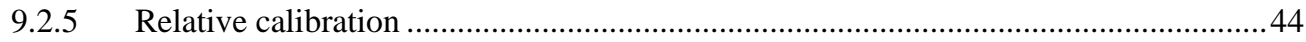

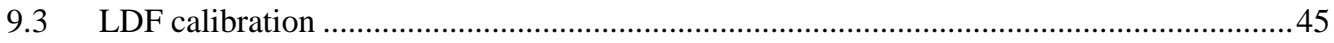

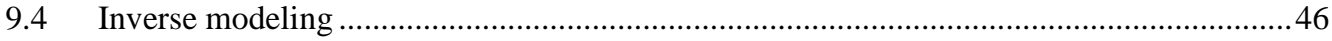

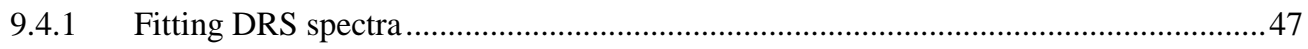




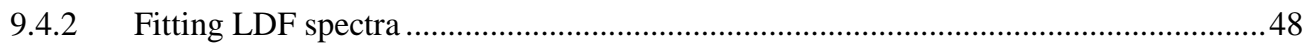

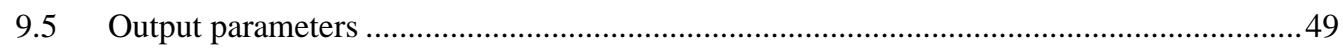

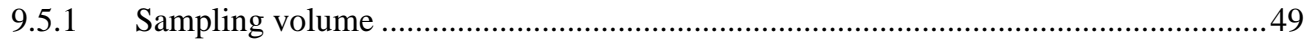

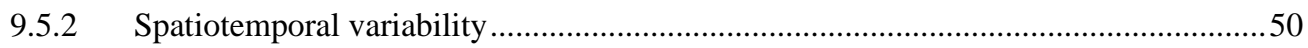

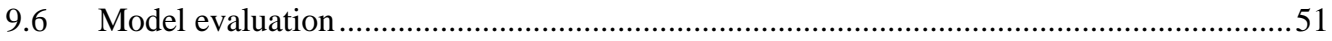

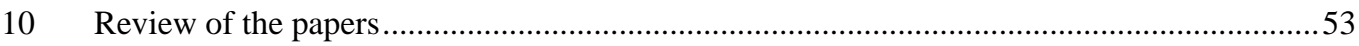

10.1 Paper I - Can a one-layer optical skin model including melanin and inhomogeneously distributed blood explain spatially resolved diffuse reflectance spectra?

10.2 Paper II - Inverse Monte Carlo for estimation of scattering and absorption in liquid optical phantoms

10.3 Paper III - Microcirculation assessment using an individualized model for diffuse reflectance spectroscopy and conventional laser Doppler flowmetry....

10.4 Paper IV - Oxygen saturation, red blood cell tissue fraction and speed resolved perfusion A new optical method for microcirculatory assessment

10.5 Paper V - Type 2 diabetes is associated with impaired microvascular function in the skin

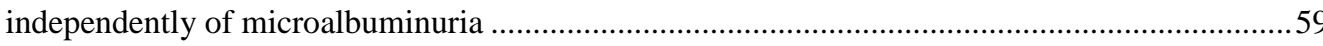

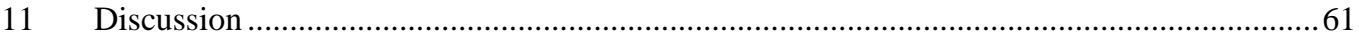

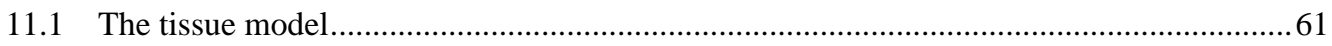

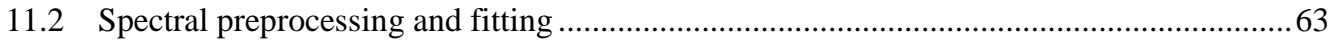

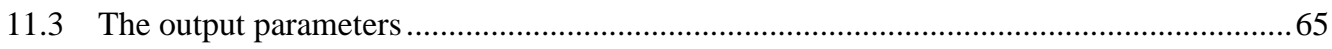

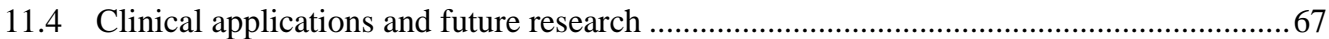

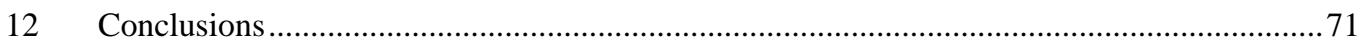





\section{INTRODUCTION}

The microcirculation, i.e. the circulation of the blood in the smallest vessels, supplies all cells in the body with oxygen and nutrients through a complex network and is essential for our body. The main transfer of oxygen and nutrients from the blood to the cells occurs in the capillaries and they are therefore denoted exchange vessels. All capillaries are not perfused all the time, and in an event of increased metabolic demand the number of perfused capillaries can increase allowing for a larger oxygen exchange, called capillary recruitment [1]. The arterioles deliver blood to the capillaries and the blood flow is regulated with smooth muscle cells causing the vessels to contract or dilate. The venules drain capillaries of blood leading it to veins. Without a well-functioning microcirculation, the metabolic demand of the surrounding tissue can become larger than the supply, leading to tissue hypoxia and cell death.

There are several diseases associated with an impaired microcirculation such as peripheral vascular disease, diabetes mellitus and hypertension [2-5]. An impaired microcirculation is also considered to be a cardiovascular risk factor [2]. To assess microcirculatory function in the body, the cutaneous microcirculation can be investigated since it is easy accessible and its function can be seen as a representative for the microcirculation in the whole body [6, 7]. In diabetes mellitus, an impaired skin microcirculation can be manifested in several ways, including impairment in the capillary recruitment or a reduced ability of the vessels to dilate when needed [8].

Several optical methods exists for non-invasive monitoring of microcirculatory blood flow or oxygen saturation. Techniques based on optical microscopy like videocapillaroscopy and sidestream darkfield (SDF) microscopy are used to visualize the microvascular network in the tissue [9, 10]. Hence, the perfused vessels can be directly visualized. However, calculations of microcirculatory flow from SDF images require image processing and manual classifications of blood vessels, making it difficult for real time analysis [10, 11]. The laser Doppler flowmetry (LDF) technique is frequently used to estimate the microvascular perfusion both in a single point using a fiber optic probe and in a larger surface using scanning LDF $[9,11]$. The laser speckle contrast analysis is a related but different technique for analyzing skin perfusion maps. Drawbacks are the use of arbitrary units in conventional LDF, which complicates between subjects and between sites comparisons. Spectroscopic technique like diffuse reflectance spectroscopy (DRS), near-infrared spectroscopy (NIRS) and diffuse correlation spectroscopy can be used to assess blood fraction and oxygen saturation in the microcirculation [11-13]. 
By simultaneously measuring blood flow and oxygen saturation, a more comprehensive assessment of the microcirculation and the metabolism can be acquired. A combination of DRS and LDF to assess oxygen saturation and perfusion in skin simultaneously has been proposed by others $[14,15]$. In these combined implementations the perfusion is estimated in arbitrary units and the two modalities are not using a common multi-layer tissue model. By including tissue modeling, calibration of spectra and Monte Carlo simulation of the light propagation in tissue, the perfusion, the blood fraction and the oxygen saturation can be estimated in absolute units [16, 17]. Additionally, a more detailed description of the blood flow in the microcirculation is obtained since the perfusion estimate can be separated into different speed regions $[16,18]$. By combining the two techniques into one integrated multilayer tissue and light transport model, it is possible to use the strengths in e.g. the scattering estimation by the DRS technique in the assessment of the perfusion by the LDF technique.

This thesis describes the development and the evaluation of an optical system for simultaneously measuring oxygen saturation, tissue fraction of red blood cells and speed resolved perfusion in absolute units. The system integrates DRS and LDF in one multimodal, multilayer skin model to individually assess oxygen saturation and blood flow by an inverse Monte Carlo technique. 


\section{AIM OF THE THESIS}

The aim was to develop and evaluate a fiber-optic probe based system for quantifying skin microcirculatory blood flow and oxygen saturation. This includes

- integrating diffuse reflectance spectroscopy (DRS) and laser Doppler flowmetry (LDF) using an individually adaptive multilayer skin light transport model

- in-vivo quantification of oxygen saturation, red blood cell tissue fraction and speed resolved perfusion, simultaneously and in absolute units.

- clinically evaluating skin microcirculation in healthy subjects and in patients with diabetes type 2 . 


\section{SKIN MICROCIRCULATION}

The skin is the largest organ in the body with function to protect the body, assist in the regulation of body temperature and detect sensory information. The skin also synthesizes vitamin D when exposed to sunlight [19]. The thickness of the skin varies with body site, where the thinnest skin is found on the eyelids $(0.5 \mathrm{~mm})$ and the thickest on the heels $(4.0 \mathrm{~mm})$. However, most of the skin is $1-2 \mathrm{~mm}$ thick [19].

There are two different structures in the skin, the epidermis and the dermis. A schematic illustration of the skin is given in Figure 3.1. The outermost layer is the epidermis which is a thin layer without any blood vessels. The epidermis consists mostly of keratinocytes, but includes also other types of cells e.g. melanocytes (melanin producing cells). The keratinocytes form a number of distinct layers in the epidermis, where most of the body have a four layered epidermis [19]. The deepest layer of epidermis is the stratum basale. Cells in the stratum basale are supported by blood from the demis, while cells in the other layers of epidermis have no blood support [19]. The epidermal thickness varies with body site typically in the range of $60 \mu \mathrm{m}$ to $300 \mu \mathrm{m}$ [20], and has also been shown to vary with age [21].

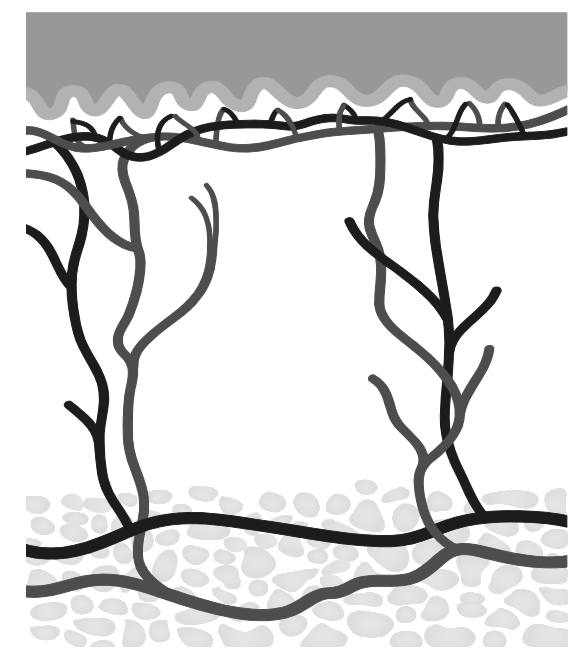

Figure 3.1. Schematic illustration of skin structure.

Below the epidermis lies the dermis, consisting mostly of connective tissue. Blood vessels, nerves, hair follicles and glands are located in the dermis. The dermis can be divided into two regions; the upper papillary region and the lower reticular region. Most of the microvasculature is located in the 
papillary region (terminal arterioles, capillaries and postcapillary venules), where the capillary loops are formed [22]. The reticular region attaches to the subcutaneous layer and consists mostly of collagen and elastic fibers [19]. Blood vessels found in the reticular dermis are arterioles and venules, generally with a diameter less than $100 \mu \mathrm{m}$ [22].

\subsection{THE MICROCIRCULATION}

The cardiovascular system distributes blood to all tissues in the body. It delivers oxygen and nutrition through a complex system of blood vessels, branching into smaller and smaller vessels from the aorta to arteries and arterioles and into the smallest vessels; the capillaries. The main exchange of oxygen, nutrients and metabolites between the blood and tissue cells takes place in the capillary network and to facilitate the exchange, the capillary wall consist of only one layer of endothelial cells and a basement membrane. The capillaries are drain into venules, further into veins and finally returning to the heart via vena cava. An overview of the average diameter, cross-sectional area and blood flow velocity for different vessel types is given in Table 3.1 .

Table 3.1. Average diameter, cross-sectional area and blood flow velocity for different vessel types [19, 23].

\begin{tabular}{l|lll}
\begin{tabular}{l} 
Vessel type \\
\multicolumn{1}{l}{ Dorta }
\end{tabular} & Diameter $[\mathrm{mm}]$ & $\begin{array}{l}\text { Cross-sectional area } \\
{\left[\mathrm{cm}^{2}\right]}\end{array}$ & Velocity $[\mathrm{mm} / \mathrm{s}]$ \\
\hline Small arteries & $0.1-10$ & $3-5$ & 400 \\
Arterioles & $0.01-0.1$ & 20 & 40 \\
Capillaries & $0.004-0.01$ & 40 & 20 \\
Venules & $0.01-0.1$ & $4500-6000$ & 0.1 \\
Small veins & $0.1-0.1$ & 250 & 3.3 \\
Vena cava & $20-35$ & 80 & 10 \\
& & 14 & 150
\end{tabular}

The microcirculation includes the smallest vessels in the circulatory system $(<100 \mu \mathrm{m}$ in diameter $)$ and comprises arterioles, venules and capillaries. The blood flow into the capillaries can be regulated by the contraction or relaxation of smooth muscle cells in the arteriole walls (vasoconstriction and vasodilation). By changing their diameter, the arterioles decrease or increase the blood flow into the capillaries. Normally, arterioles change diameter spontaneously 5-10 times per minute called vasomotion [19]. The regulation of smooth muscle cells can be controlled by different agents such as nitric oxide (NO), endothelium-derived hyperpolarizing factors (EDHF) and prostanoids [24]. With endothelial dysfunction, the smooth muscle cells lose their ability to relax sufficient and causing an impaired vasodilatation. Endothelial dysfunction has been associated with cardiovascular risk factors [3], and is observed in several diseases such as diabetes [25] and atherosclerosis [26]. 


\subsection{MICROVASCULAR REACTIVITY TESTS}

To assess the skin microcirculatory status using laser Doppler flowmetry and reflectance spectroscopy, the reactivity of the microvessels after a microcirculatory provocation is usually studied $[9,24,27]$. The most common microvascular tests are thermal provocations, post-occlusive reactive hyperemia and iontophoresis. Thermal provocation and systolic occlusion are tests used in this thesis and they are therefore described in more detail in the following subchapters. Other tests are limb position changes, breathing tests and venous occlusion [28]. Controlled environmental temperature is important for all tests, since skin temperature affects the blood flow [29].

\subsubsection{Thermal test}

When heating the skin locally, the response in blood flow is typically an initial peak during the first 10 minutes followed by a nadir. A plateau is reached after 20-30 minutes of heating [24]. A schematic response in perfusion during local heating is depicted in Figure 3.2. Maximal vasodilatation is obtained with a temperature of the heater between 42 and 44 degrees and a reduced maximal blood flow at plateau is associated with several diseases, including diabetes [30, 31]. The first peak is a response in blood flow mostly caused by local sensory nerves, while the plateau is mostly caused by NO [32].

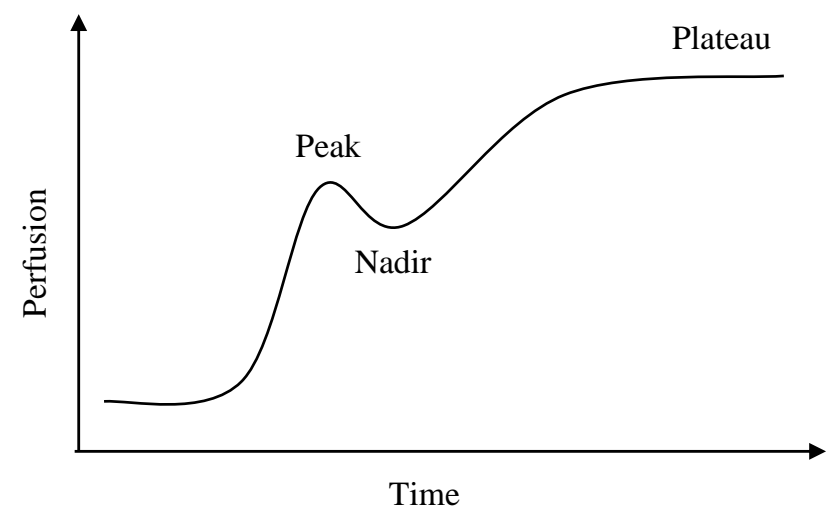

Figure 3.2. Schematic response in perfusion during local heating

\subsubsection{Systolic occlusion}

Post-occlusive reactive hyperemia (PORH) is referred to as the microvascular response upon release after an arterial occlusion [33]. After a period of ischemia, the reperfusion is characterized by a rapid peak in blood flow, slowly returning to baseline values, see Figure 3.3. The oxygen saturation is reaching a plateau after release of the occlusion, and the return to baseline values are slower than for the perfusion. Systolic occlusion of the arm can be achieved by placing a cuff on the upper arm and 
increase the pressure to above the systolic blood pressure $[33,34]$. The period of occlusion can vary, but commonly used periods are 3 to 5 minutes. The perfusion increase from baseline is correlated to the ischemic period $[33,34]$.
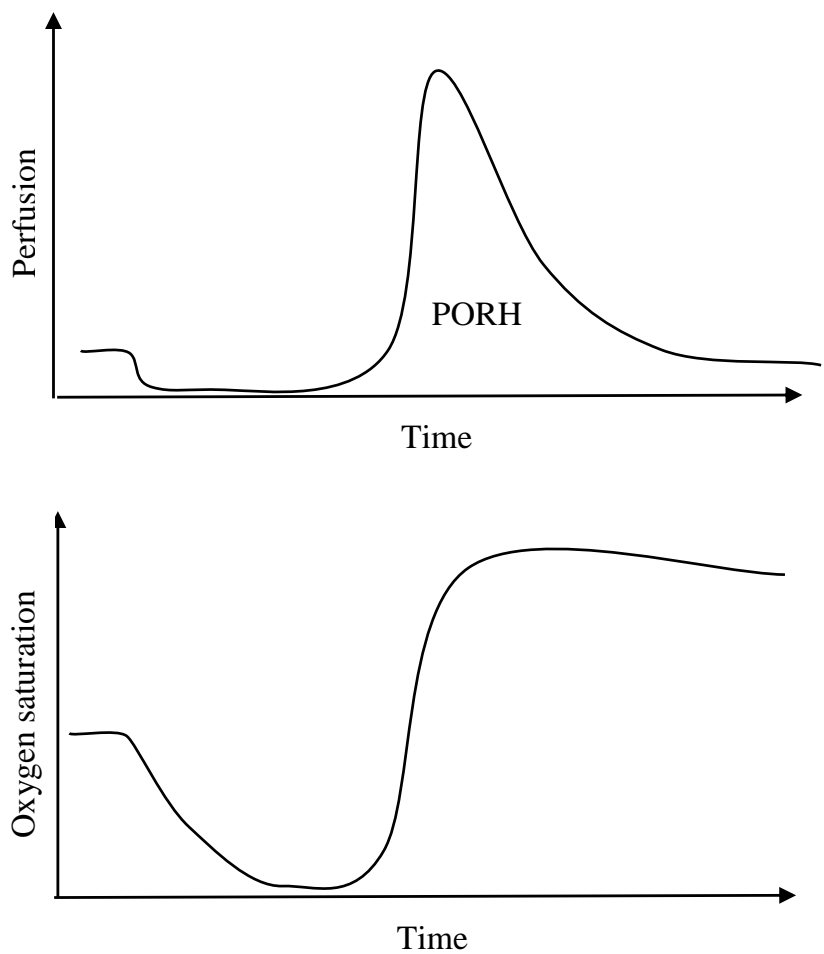

Figure 3.3. Schematic response in perfusion and oxygen saturation after an arterial occlusion.

In patients with peripheral arterial disease (PAD), the time to reach a maximal blood flow is prolonged and the perfusion increase from baseline values is reduced [35]. Strain et al. [36] reported an abnormal reperfusion pattern, a rapid early peak in perfusion after occlusion, related to cardiovascular risk. Adingupu et al. [27] added measurements on oxygen saturation and showed an altered oxygen saturation seen as a result from the abnormal reperfusion found in [36]. 


\section{OPTICAL PROPERTIES OF TISSUE}

Light is an essential aid in the field of medicine. It can be used in numerous applications including diagnostics, monitoring, surgery and therapeutic applications. Light propagation in biomedical optics can be described by a wave and a particle representation. When consider light as an electromagnetic wave, light propagation can be described mathematically by Maxwell's equations [37]. Light can also be seen as composed of particles (photons) with an amount of energy, $E$, proportional to the frequency, $f: E=h f$ where $h$ is Planck's constant. The frequency is expressed by $f=c / \lambda$ where $c$ is the speed of light and $\lambda$ is the photon wavelength.

In tissue, light propagation will be effected by the optical properties of the tissue under investigation. On the surface of tissue and also inside the tissue volume light is reflected and refracted, described by the refractive index. Molecules in tissue can absorb photons and can also in some cases emit light with a different wavelength than the absorbed photon wavelength (luminance). Luminance has the main forms of fluorescence or phosphorescence. In fluorescence, the emitting of a photon of a different wavelength occurs several ns after the absorption event, while the time delay in phosphorescence is in the order of $\mathrm{ms}$ [38]. Scattering particles cause photons to change their direction and the scattering process can be elastic or inelastic. In elastic scattering there is no energy transfer between photon and scattering particle, while in inelastic scattering the photon can both gain or lose energy [38]. Quasi-elastic scattering is a form of elastic scattering, but the frequency of light is slightly broaden due to Doppler shifts. The optical properties of tissue are usually described by the refractive index of the tissue $(n)$, the absorption coefficient $\left(\mu_{a}(\lambda)\right)$, the scattering coefficient $\left(\mu_{s}(\lambda)\right)$ and the scattering phase function $p(\theta)[39,40]$.

\subsection{REFRACTIVE INDEX}

The refractive index $n[-]$ is the ratio between the speed of light in vacuum and the speed of light in the medium, $v$, according to

$$
n=\frac{c}{v}
$$

A refractive index mismatch between two medium, $n_{1} \neq n_{2}$, will cause a deviation in the original photon path. The angle of refraction $\theta_{2}$ related to the incident angle $\theta_{1}$ is described by Snell's law: 


$$
n_{1} \sin \theta_{1}=n_{2} \sin \theta_{2}
$$

\subsection{ABSORPTION}

Absorption is a process where the photon energy is transferred to the tissue and transformed to other forms of energy, for example heat or fluorescence. The molecule absorptivity is determined by the molecular structure and is wavelength dependent. Therefore, molecules can be identified by their unique absorption spectrum, using e.g. DRS [41-43].

The absorption efficiency of a single absorbing particle is described by the effective cross-section area, $\sigma_{a}\left[\mathrm{~mm}^{2}\right]$. The effective cross-section area is dependent both on the actual size of the absorbing particle $\left(A_{a}\right)$ and also on the light absorption efficiency of the particle $\left(Q_{a}\right)$ :

$$
\sigma_{a}=A_{a} Q_{a}
$$

In a volume with multiple absorbers, the product of the volume density of absorbers $\rho_{a}\left[\mathrm{~mm}^{-3}\right]$ and the effective cross-section of the absorbers gives the absorption coefficient $\mu_{a}\left[\mathrm{~mm}^{-1}\right]$ :

$$
\mu_{a}=\rho_{a} \sigma_{a} .
$$

In a non-scattering medium, the light intensity decrease per light path length $d[\mathrm{~mm}]$ due to the absorption property $\mu_{a}$ is given by the Beer-Lambert law:

$$
I(d)=I_{0} e^{-\mu_{a} d},
$$

where $I_{0}$ is the incident light intensity.

\subsubsection{Skin chromophores}

Absorbers in the skin are referred to as chromophores. The two most dominant chromophores in the skin in the visible wavelength range are melanin and hemoglobin [44], Figure 4.1. Other absorbers contributing less to the total absorptivity of the skin in the visible range are bilirubin, carotene, lipids and water [39, 44, 45]. Bilirubin and beta-carotene absorption dominate below $500 \mathrm{~nm}$ and have absorption maxima around $450 \mathrm{~nm}$ [39]. Water and fat have absorption peaks above $900 \mathrm{~nm}$ and the contribution in the wavelength range 450 to 850 is weak. 


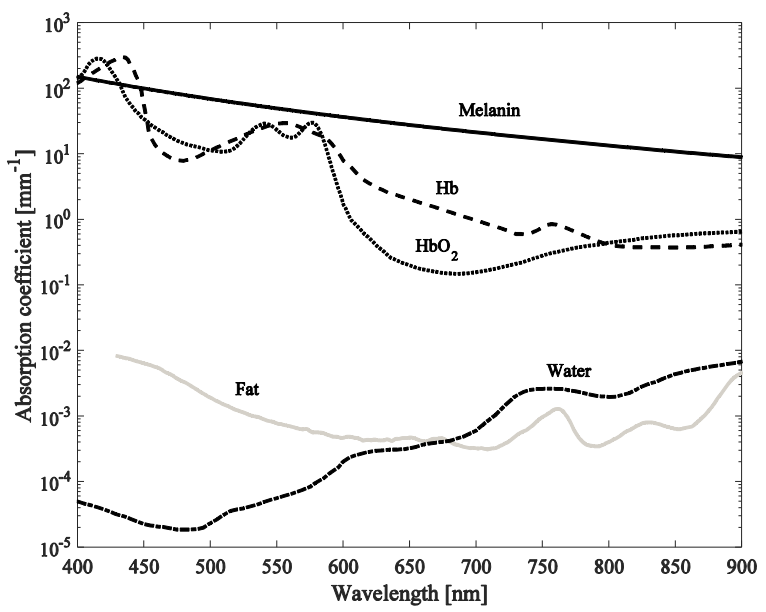

Figure 4.1. Absorption coefficient for hemoglobin ( $\mathrm{HbO}_{2}$, oxygenized and $\mathrm{Hb}$, deoxygenized hemoglobin respectively) [46], melanin [47], water [48] compiled by Prahl at http://omlc.org/spectra/water/abs/index.html and fat [49] compiled by Prahl at http://omlc.org/spectra/fat/.

\section{Melanin}

Melanin is found in the outermost layer of the skin, the epidermis, and is produced be the melanocytes cells. There are two forms of melanin with different absorption spectra; pheomelanin and eumelanin, see Figure 4.2. Pheomelanin is red or yellow and eumelanin is black or brown. The ratio between these two pigments can be seen in skin and hair color, where individuals with red hair and light skin individuals have more pheomelanin than those with dark hair and dark skin [50]. However, eumelanin is the most common form of melanin in the epidermis for all skin types [51]. Differences in skin color depends also on the produced amount of melanin, where individuals with dark skin have more melanin in the epidermis than those with light skin. By exposing skin to UV radiation, the melanin darkening and the production of melanin increases, causing the skin to tan [52]. The fraction of melanin in the epidermis varies from about $1-6 \%$ in light skin up to $18-43 \%$ in dark skin $[53,54]$. 


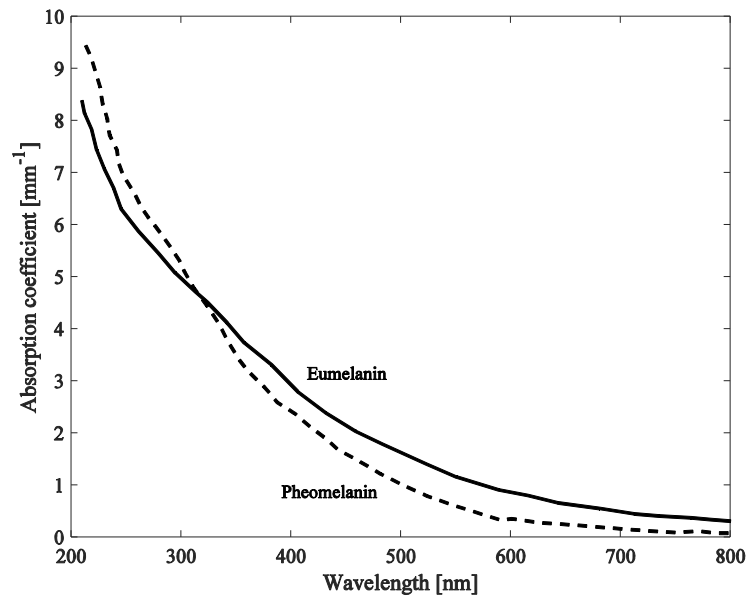

Figure 4.2. Absorption coefficient for $2 \%$ eumelanin (solid) and $2 \%$ pheomelanin (dashed), data compiled by Jacques [55].

\section{Hemoglobin}

Hemoglobin is located in the red blood cells and carry four oxygen molecules from the lungs to the microcirculation where it may be released to the cells. Oxygenized and deoxygenized hemoglobin have characteristic absorption spectra, oxygenized hemoglobin has two peaks in the visible region at 542 and 577 and deoxygenized hemoglobin has one peak at $554 \mathrm{~nm}$, see Figure 4.3.

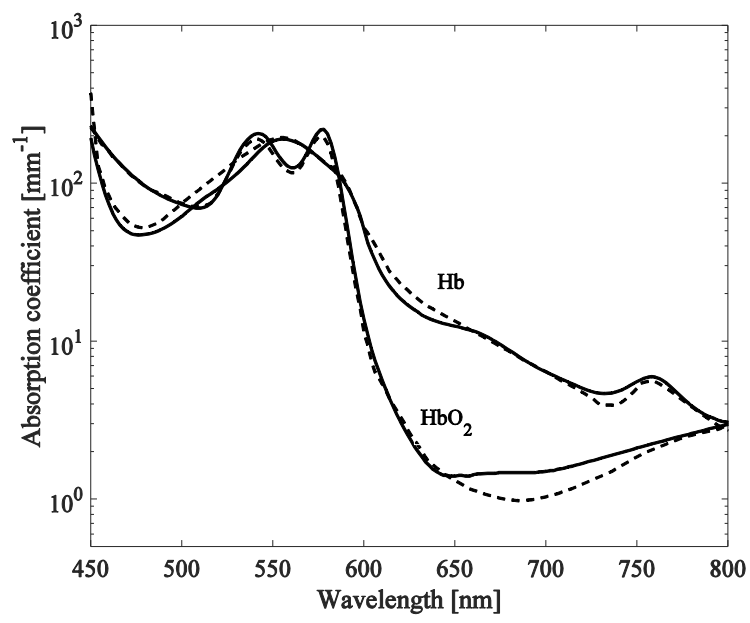

Figure 4.3. The absorption coefficient in the visible range for oxygenized and deoxygenized hemoglobin, $\mathrm{HbO}_{2}$ and $\mathrm{Hb}$ respectively. Compiled by Prahl [46](dotted) and Zijlstra [56](solid). 


\subsection{SCATTERING}

Scattering is a change in direction of the photon due to an interaction with a small particle or molecule, and is dependent on the wavelength of the incident light, the size of the scattering particle and the refractive index mismatch [57]. As for absorption, scattering efficiency of a single particle is given by the effective scattering cross section area, $\sigma_{s}=Q_{s} A_{s}$, and in a medium with multiple scattering particles, the scattering coefficient $\mu_{s}\left[\mathrm{~mm}^{-1}\right]$ is given by:

$$
\mu_{s}=\rho_{s} \sigma_{s}
$$

where $\rho_{s}$ is the volume density of the scattering particles in the medium. The inverse of the scattering coefficient is called the mean free path $\left(m f p_{s}=1 / \mu_{s}\right)$ and reflects the average distance a photon travels before being scattered.

The scattering particles in the tissue differ in sizes and photons are mostly scattered by particles with sizes matching the photon wavelength [58]. The largest scattering structures are cells and nuclei with a diameter of approximately $10 \mu \mathrm{m}$. Smaller structures are mitochondria and collagen fibers, and the smallest are membranes with a diameter of about $0.01 \mu \mathrm{m}$ [59]. Light scattered by particles with the same size or larger than the wavelength $\lambda$ is referred to as Mie scattering. When the scattering particles are smaller than the wavelength of the incident light (less than about $\lambda / 15$ ), the scattering can be described by Rayleigh scattering [60], which is the Rayleigh limit of Mie scattering. In Rayleigh scattering, the scattering cross section area is strongly wavelength dependent by $\lambda^{-4}$.[57]

The scattering in the epidermis is mainly due to the melanocytes while in the lower skin structure, the dermis, collagen is the largest contributor [44].

\subsubsection{Scattering phase functions}

If a scattered photon changes its path with a deflection angle $\theta$, see Figure 4.4,

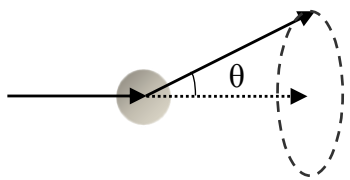

Figure 4.4. Scattering from a single scattering particle 
the scattering phase function $p(\theta)$ describes the probability distribution of all possible scattering angles in the medium. The phase function is normalized to one, i.e. the total probability over all angles is equal to one:

$$
2 \pi \int_{0}^{\pi} p(\theta) \sin \theta d \theta=1 .
$$

The distribution of scattering angles can also be described by the anisotropy factor, $g$, which is defined as the expectation value of cosine of the scattering angle

$$
g=2 \pi \int_{0}^{\pi} p(\theta) \cos \theta \sin \theta d \theta=\langle\cos \theta\rangle
$$

by using equation (4.7).

A value of $g=0$ indicates equal probability of scattering forward as backward, and values near 1 indicates highly forward scattering, see Figure 4.5. In tissue, $g$ values range approximately between 0.75 and 0.98 [40], which is mostly forward scattering.

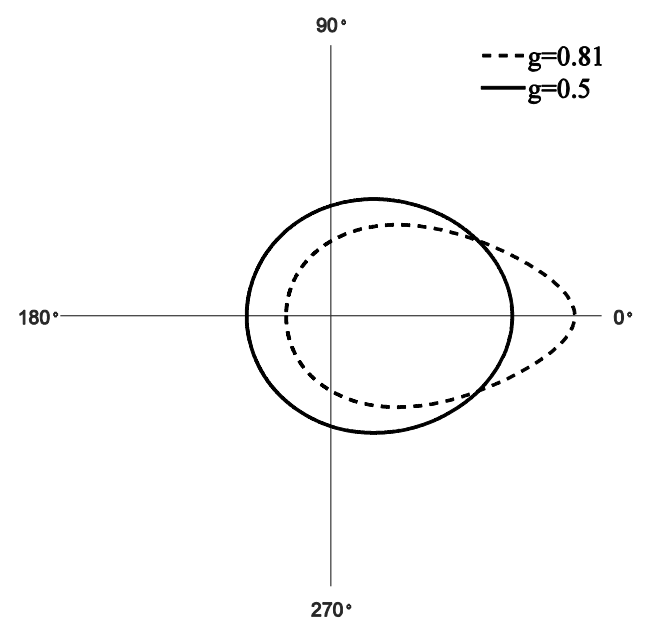

Figure 4.5. 2D polar plot of a Henyey-Greenstein phase function for $g=0.5$ (solid) and $g=0.81$ (dotted)

If the size and the refractive index of the scattering particles are known, and the assumption that they are spherical and homogenously distributed in the media holds, Mie theory can be used to calculate the phase function. In tissue however, the size and the refractive index of the scattering particles differ. 
Hence, a distribution of sizes and refractive indices needs to be handled, making Mie theory calculations complex [44].

\section{Henyey-Greenstein phase function}

An approximation of the phase function for human skin is the Henyey-Greenstein phase function, originally developed to describe interstellar scattering [61]. The phase function is given by

$$
p_{H G}(\theta)=\frac{1}{4 \pi} \frac{1-g^{2}}{\left(1+g^{2}-2 g \cdot \cos (\theta)\right)^{3 / 2}}
$$

where $g$ is the anisotropy factor.

\section{Gegenbauer kernel phase function}

The two-parametric Gegenbauer kernel phase function to describe scattering in biological tissue was introduced in 1980 by Reynolds and McCormick [62] according to

$$
p_{G K}(\theta)=\frac{\alpha_{G K} g_{G K}\left(1-g_{G K}\right)^{2 \alpha_{G K}}}{\pi\left(\left(1+g_{G K}\right)^{2 \alpha_{G K}}-\left(1-g_{G K}\right)^{2 \alpha_{G K}}\right)} \cdot \frac{1}{\left(1+g_{G K}^{2}-2 g_{G K} \cos \theta\right)^{\alpha_{G K}+1}} .
$$

The phase function in Equation (4.10) includes two parameters; $\alpha_{G K}$ and $g_{G K}$, where $g_{G K} \neq g$ for all cases except at $\alpha_{G K}=1 / 2$. When $\alpha_{G K}=1 / 2$, Equation (4.10) becomes the Henyey-Greenstein phase function. The Gegenbauer kernel phase function has been found to better fit scattering from smaller particles (3-240 $\mu \mathrm{m}$ in diameter) with higher relative index of refraction (1.015-1.25), compared to the Henyey-Greenstein phase function [62].

\subsubsection{The reduced scattering coefficient}

In a highly forward scattering medium it takes many scattering events before photons are diffuse, i.e. have a random direction. The reduced scattering coefficient $\mu_{s}^{\prime}\left[\mathrm{mm}^{-1}\right]$, a combination of the scattering coefficient and the anisotropy factor, can therefore be used to describe scattering in tissue

$$
\mu_{s}^{\prime}=\mu_{s}(1-g)
$$

Many small steps of length $1 / \mu_{s}$ and scattering angles $\theta$ can then be described by larger step of $1 / \mu_{s}^{\prime}$ and the medium can be considered isotropic with reduced scattering coefficient $\mu_{s}^{\prime}$, depicted in Figure 4.6. 


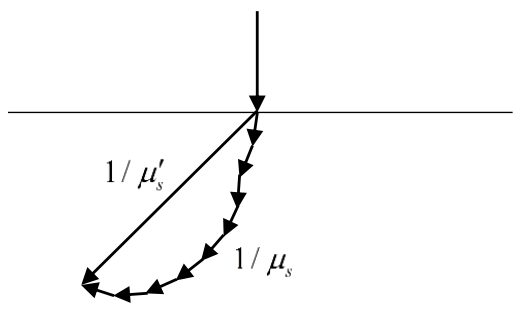

Figure 4.6. Schematic illustration of the relationship between small steps of $1 / \mu_{s}$ and a large step of $1 / \mu_{s}^{\prime}$.

\subsection{THE TOTAL ATTENUATION COEFFICIENT}

The combination of the absorption and scattering coefficient is described by the total attenuation coefficient:

$$
\mu_{t}=\mu_{a}+\mu_{s}
$$

\subsection{THE ALBEDO}

The albedo is defined as the ratio between the scattering coefficient and the total attenuation coefficient:

$$
\text { albedo }=\frac{\mu_{s}}{\mu_{s}+\mu_{a}}=\frac{\mu_{s}}{\mu_{t}} .
$$




\section{LASER DOPPLER FLOWMETRY}

Laser Doppler flowmetry (LDF) is a technique for estimating blood flow in tissue. When laser light is scattered by a moving red blood cell (RBC) or other moving cells in the tissue, a small frequency shift in the light arises (Doppler shift). By illuminating the tissue with monochromatic light and analyzing the frequency distribution of the backscattered light via heterodyne mixing on a detector, a perfusion measure (the fraction of RBCs times their average speed), can be calculated.

\subsection{SingLE DOPPLER SHIFT}

When light is scattered by a moving object it will change its frequency. This frequency shift is i.e. Doppler shift, occurs for all wave propagation. The size of a single Doppler shift $\Delta f$ (the difference between incident frequency $f_{i}$ and scattered frequency $f_{s}$ ) can be calculated from the scalar product between the scattering vector $\mathbf{q}$ and the velocity vector of the scattering particle $\mathbf{v}$ :

$$
\Delta f=f_{i}-f_{s}=\mathbf{v} \cdot \mathbf{q} .
$$

The scattering vector $\mathbf{q}$ is the difference between the incoming vector $\mathbf{k}_{i}$ and the scattered vector $\mathbf{k}_{s}(|\mathbf{k}|=1 / \lambda)$

$$
\mathbf{q}=\mathbf{k}_{i}-\mathbf{k}_{s}=\frac{2}{\lambda} \sin \frac{\theta}{2} .
$$

The angle $\varphi$ is the angle between $\mathbf{q}$ and $\mathbf{v}$, see Figure 5.1.

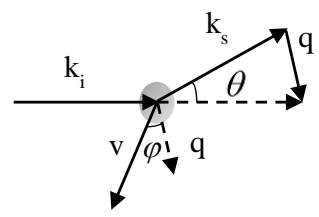

Figure 5.1. A single Doppler shift by a moving scattering With Equation (5.1) and (5.2), the frequency shift can be expressed by 


$$
\Delta f=2 \frac{v}{\lambda} \sin \frac{\theta}{2} \cos \varphi
$$

As seen in Equation (5.1), a single Doppler shift scales linear to the velocity of the moving RBCs.

The maximal Doppler shift occurs when $\sin \frac{\theta}{2}$ is at maximum (Equation (5.2)), i.e. $\theta=\pi$ (backward scattering). However, RBCs are extremely forward scattering ( $g \approx 0.99$ [40]) and $\theta$ will therefore mostly be close to zero. When $\theta$ is close to zero, the scattering vector $\mathbf{q}$ will be orthogonal to the incoming vector $\mathbf{k}_{\mathbf{i}}$ and maximal Doppler shift will then occur when the direction of the RBC is orthogonal to the direction of light, i.e. $\cos \varphi=1$.

An analytic calculation of single velocity Doppler histogram can be performed by using the Gegenbauer kernel phase function (section 4.3.1) [63].

\subsection{DOPPLER SPECTRUM}

All monochromatic laser light, both shifted and non-shifted will interfere on the detector, forming a speckle pattern on the detector. This speckle pattern will fluctuate due to the differences in frequencies (if there is no frequency differences, i.e. no Doppler shifts, the speckle pattern is static). The link between the optical Doppler spectrum, $I(f)$, based on individual Doppler shift, and the power spectral density of the detector current, called the Doppler power spectrum, was described by Forrester in 1961 [64]. The current generated from the detector $i(t)$ will vary over time and include one static part $i_{d c}$ and one time varying part $i_{a c}$. The static part of the detector current is calculated as [65]:

$$
i_{d c}=\int_{-\infty}^{\infty} I(f) d f
$$

The Doppler power spectrum is the power spectral density of the time varying detector current and is calculated as the autocorrelation of the optical Doppler spectrum [65] according to

$$
P(f)=P S D\left(i_{a c}\right)=2 \cdot(I \bullet I)(f),
$$

where $\bullet$ denotes the autocorrelation.

The Doppler power spectrum will be affected by the RBC tissue fraction and the speed of the RBCs. An increase in RBC tissue fraction will cause an increase in power in the Doppler power spectrum for all frequencies. However at high RBC tissue fractions, the spectrum will instead broaden due to multiple shift, Figure 5.2a. An increased average speed of the RBCs will cause a broadening of the Doppler power spectrum Figure 5.2b. 

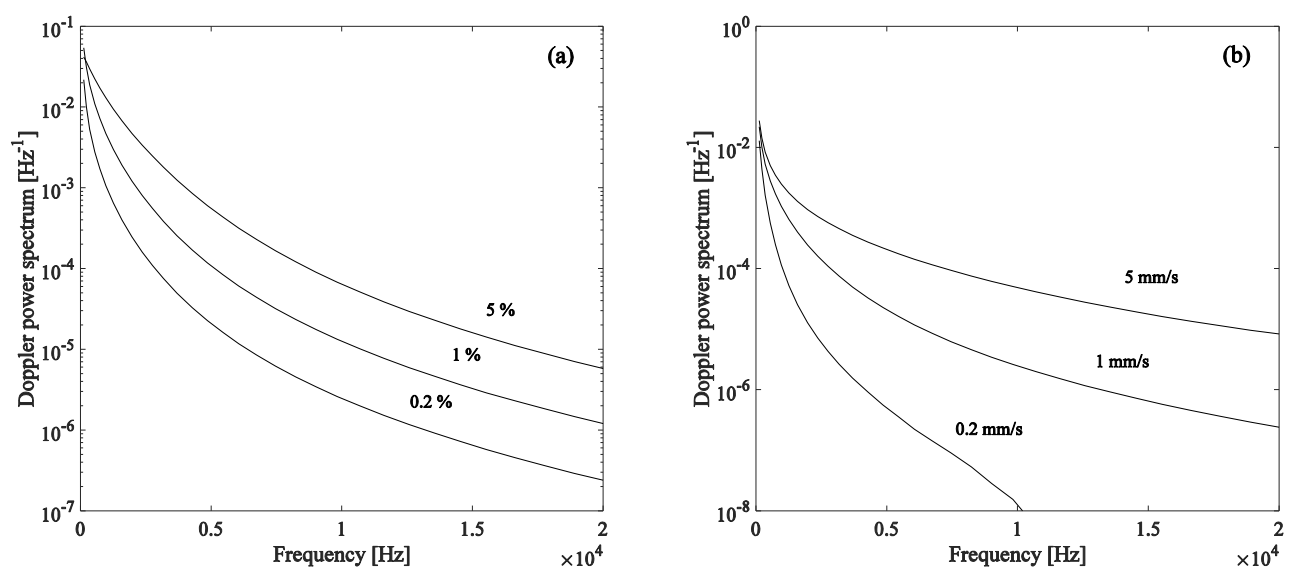

Figure 5.2. Changes in Doppler power spectrum due to different RBC tissue fractions (a) and average $R B C$ speeds $(b)$.

The Doppler power spectrum is simulated with an oxygen saturation of $40 \%$ and an epidermal thickness off $75 \mu \mathrm{m}$. In Figure 5.2a. the RBC speed is $1 \mathrm{~mm} / \mathrm{s}$ and in Figure 5.2b. the tissue fraction of $\mathrm{RBC}$ is $0.2 \%$.

\subsection{CONCENTRATION OF RED BLOOD CELLS AND PERFUSION}

The concentration of moving red blood cells (CMBC) and conventional perfusion can be estimated from the Doppler power spectrum [66]. The CMBC scales to the fraction of moving red blood cells in the tissue and the conventional perfusion scales to the tissue fraction of RBCs times their average speed.

The CMBC is calculated as the zero moment of the Doppler spectrum, normalized with the static current on the detector $i_{d c}$, according to:

$$
C M B C=\frac{\int_{-\infty}^{\infty} P(f) d f}{i_{d c}^{2}},
$$

and conventional perfusion is calculated as the first moment of the Doppler spectrum, normalized with $i_{d c}$

$$
\text { Perfusion }=\frac{\int_{-\infty}^{\infty} f \cdot P(f) d f}{i_{d c}^{2}} .
$$


Drawbacks of both the $\mathrm{CMBC}$ and the conventional perfusion are the arbitrary units of the estimates. They also scales nonlinearly with the tissue fraction of RBCs, especially the CMBC, leading to underestimated values at high RBC tissue fractions [67]. 


\section{OPTICAL PHANTOMS}

Optical phantoms can be used to mimic light transport in tissue. Their application areas include calibration of measurement systems and as reference objects when developing and evaluating optical methods. Depending on intended use, different characteristics of the phantom are important. For some applications stability and consistency over time are important while knowledge of the exact optical properties less relevant. For other applications, a precise theoretically prediction of the optical properties is essential. The optical properties of interest in small source-detector distance applications $\left(<1 / \mu_{s}^{\prime}\right)$ are the absorption coefficient, the scattering coefficient, the scattering phase function and the anisotropy factor [68]. For larger distances, where diffusion theory is valid, only the reduced scattering coefficient and the absorption coefficient are required to describe the light transport $[68,69]$. The optical properties of a phantom can be assessed by different techniques such as integrating sphere measurements [70], collimated transmission [71] and spatially resolved diffuse reflectance (SRDR) measurements [72].

Phantoms can exist in various types (liquid, gels, silicon-based, solids etc.), various structures (single-layer or multi-layer) and with different distribution of absorbers (homogenous or in inclusions such as vessels or flow tubes) [69]. Liquid phantoms are easy to mix, but the stability over time is limited. Solid phantoms usually have a more complex preparation process, but the stability over time can last for years [69]. A common standardization of optical phantoms and characterization of the optical properties is missing, and the application area determines which optical phantom and characterization method that is best suited.

In this thesis, liquid optical phantoms consisting of Intralipid® as scattering component and food dyes as absorbing component have been used. The absorption and scattering coefficients of the phantoms were assessed with collimated transmission measurements, described in section 6.2.1. The optical phantoms were used for evaluating different calibration procedures, scattering phase function and number of detecting fibers for DRS in paper II.

\subsection{CONSTRUCTING OPTICAL PHANTOMS}

The homogeneity of the optical properties in a layer or in a vessel of a phantom, or in the whole phantom is important. Some scattering components are hydrophobic and are not easily mixed with water based dyes. This can result in an inhomogeneous distribution of scattering and 
absorbing particles which will make the characterization of the optical phantom difficult. If the volume of scattering particles is large, a linear relationship between the scattering coefficient and the concentration cannot be assumed [73, 74]. In high density scattering media, the scattering events becomes dependent and the scattering coefficient will be lower than expected.

\subsubsection{Scattering components}

Polystyrene microspheres and Intralipid $®$ are well characterized media and commonly used as scattering components in liquid phantoms. Other used scattering components are milk and also metal oxide powders like titanium dioxide with a high scattering coefficient [69].

\section{Microspheres}

When using polystyrene microspheres, the size of the particles and the refractive index are controlled by the manufacture, with a high accuracy. Hence, the scattering can be derived theoretically by using Mie theory, assuming the particles to be homogenously distributed in the solution. Microspheres are therefore a repeatable and accurate scattering component. However the cost for microspheres is high. Microspheres also needs to be stored properly, where freezing can cause aggregation of the microspheres which will alter the measured scattering coefficient [17].

\section{Intralipid}

Intralipid ${ }^{\circledR}$ is a fat emulsion used as intravenous nutrition for humans. It consists of soy bean oil, egg phospholipids and glycerin and exists in concentrations of $10 \%, 20 \%$ and $30 \%$. The scattering of Intralipid ${ }^{\circledR}$ is somewhat similar to biological tissue since the structures in the component causing scattering is similar. Intralipid ${ }^{\circledR}$ also has a high scattering coefficient and a low absorption coefficient, which is desirable for a scattering component [75]. The optical properties of Intralipid ${ }^{\circledR}$ could vary between different batches and needs to be assessed for each batch [76]. A precise knowledge of the particle sizes and shapes in Intralipid ${ }^{\circledR}$ is necessary to be able to use Mie theory to describe the scattering. For Intralipid® ${ }^{\circledR}$ 10\%, van Staveren et al. [77] measured the particle size to be in the range of $25-675 \mathrm{~nm}$ and found the shape to be approximately spherical by electron microscopy. If the particle sizes are unknown, other approximations of the scattering phase function like Henyey-Greenstein and Gegenbauer kernel can be used to describe the scattering.

\subsubsection{Absorbing components}

Absorbing components in phantoms are commonly inks and dyes, but whole blood or hemoglobin are also used [69]. Inks and dyes can be water based or oil based, and when choosing absorbing component it is important to ensure the compatibility with the scattering component. Food dyes are easily available and can be both water based and oil based. As for 
the high scattering media, the food dyes used in paper II showed a non-linear behavior with concentration for high concentration of absorbers.

\subsection{MEASURING OPTICAL PROPERTIES OF PHANTOMS}

If the optical properties of the phantom components are unknown and not possible to derive theoretically, they need to be measured with an independent method. One such method is spectral collimated transmission measurements [71].

\subsubsection{Spectral collimated transmission}

The spectral collimated transmission (SCT) setup is illustrated in Figure 6.1. The detected light $I$ will depend on the thickness of the sample, $d$.

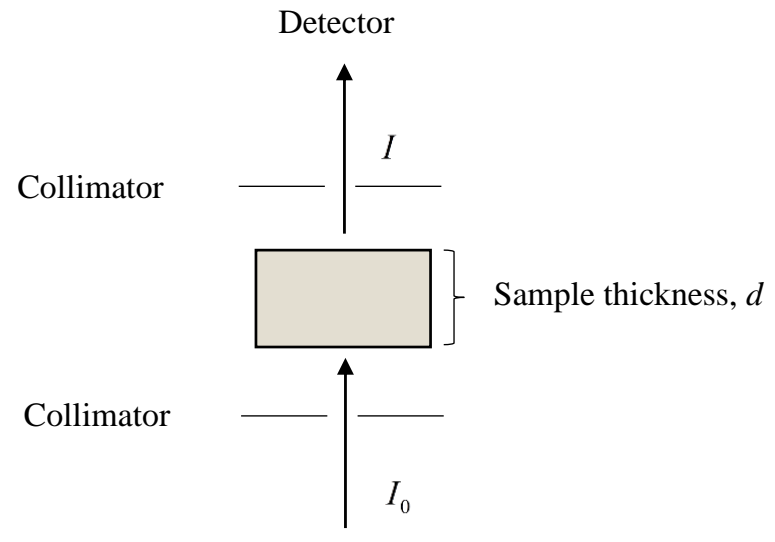

Light source

Figure 6.1. Schematic illustration of a spectral collimated transmission setup.

By measuring $I$ as function of different sample thicknesses $d$, the total attenuation coefficient of the sample can be determined by the Beer-Lambert law

$$
\mu_{t}(\lambda)=\frac{\ln \left(I_{0}(\lambda) / I(\lambda)\right)}{d}
$$

The absorption coefficient for different diluted food dyes is given in Figure 6.2, assuming the scattering coefficient to be zero. 


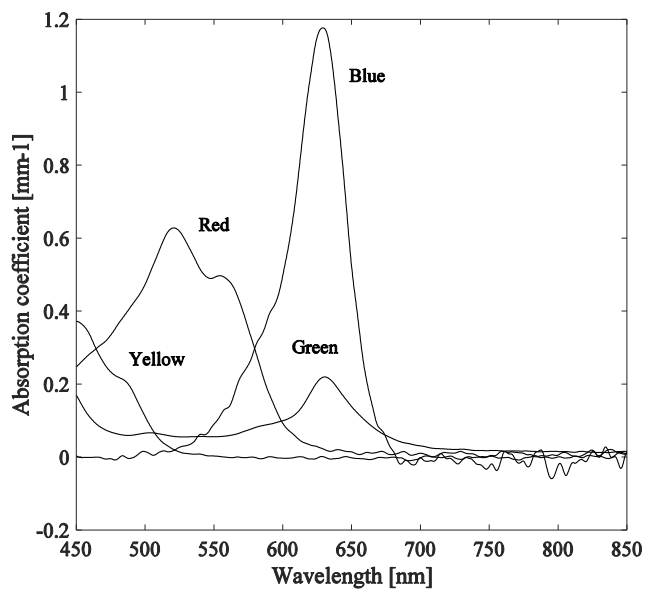

Figure 6.2. Absorption coefficient for diluted (1/100) red, blue, green and yellow food dyes measured with SCT. 


\section{Monte Carlo simulations}

Monte Carlo (MC) methods are able to simulate photon transport in biological tissues and have been described by Wang et al. [78] and Prahl et al. [79]. By setting probabilistic rules for how photons are allowed to propagate in tissue, based on optical properties, and simulating the random photon path in tissue for a large number of photons, a numerical result of the light transport is obtained. The computational speed of Monte Carlo has been a limitation of the technique, since a large number of photons is required to obtain reliable results. By using parallel computing on a graphics processing unit (GPU) [80] and post processing techniques [81-83], the computational time can be greatly reduced. Monte Carlo simulations has been widely used within biomedical optics, for instance to simulate measurements with DRS [13, 84], fluorescence spectroscopy [85], LDF [16, 86, 87], optical coherence tomography (OCT) [88] and in photodynamic therapy (PDT) [89].

\subsection{The Monte Carlo Method}

The flowchart in Figure 7.1 describes the most important steps in the Monte Carlo method for a homogenous semi-infinite medium. With $\mathrm{MC}$ it is also possible to simulate layered structures and heterogeneities like blood vessels. Other steps like boundary conditions and internal reflection are then needed to be considered. The optical properties of the medium required to simulate the photon path are the absorption coefficient $\mu_{a}$, the scattering coefficient $\mu_{s}$, the scattering phase function $p(\theta)$ and the refractive index $n$. Random numbers, $\xi$, uniformly distributed between 0 and 1 are used in several steps of the Monte Carlo method. The steps are described in the following subchapters. 


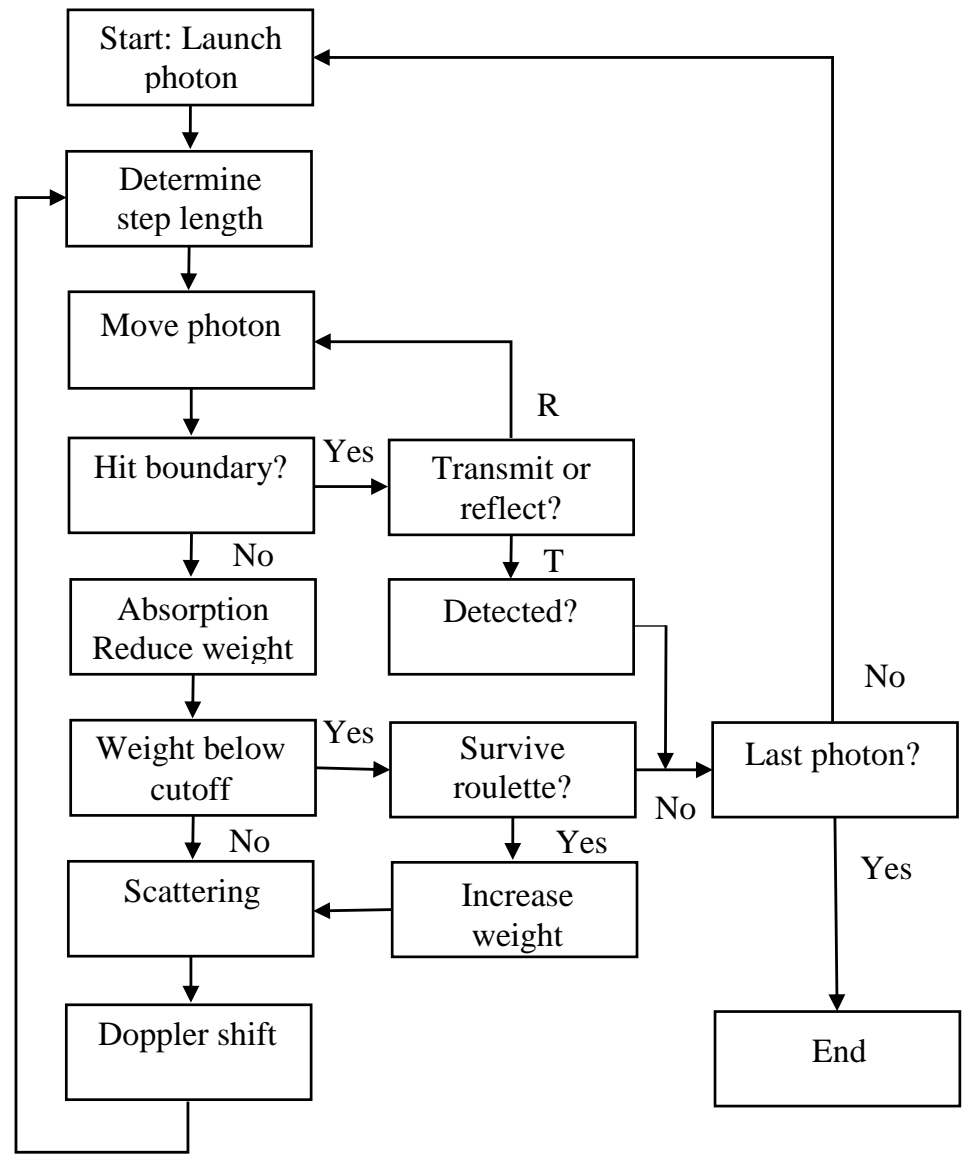

Figure 7.1. Flow chart of the Monte Carlo method to simulated photon transport in tissue.

\subsubsection{Photon launch}

Each photon is initialized with a starting position $\left(x_{0}, y_{0}, z_{0}\right)$, a direction $\left(u_{x}, u_{y}, u_{z}\right)$ and a weight (usually $w_{0}=1$ ). The starting position and the direction depend on light source properties, for example numerical aperture and radius of the fiber. A distributed light source from e.g. a fiber, can be accounted for in the simulation as described above, or in the post-processing with a pencil beam injection using convolution to account for the distributed light source [90]. The position of the photon is often assumed to follow a uniform distribution or a Gaussian distribution over the light source geometry [91]. At the border between the medium and the probe, some photons will be reflected due to the reflective index mismatch between light source and medium, and hence never propagate in the medium, see section 7.1.6. 


\subsubsection{Move photon}

To move the photon, the step length of the photon is determined by

$$
s=\frac{-\ln \xi}{\mu_{t}}
$$

where $\xi$ is random number uniformly distributed between 0 and 1 , and $\mu_{t}$ is the total attenuation coefficient, i.e. the sum of the absorption and scattering coefficient. Equation (7.1) is derived direct from the Beer-Lambert law, since the cumulative distribution function $F(s)$ for the step size can be described by

$$
F(s)=1-e^{-\mu_{t} s}
$$

and $F^{-1}(\xi)=s[78]$.

The new position for the photon can then be calculated as

$$
\begin{aligned}
& x_{1}=x_{0}+u_{x} \cdot s \\
& y_{1}=y_{0}+u_{y} \cdot s \\
& z_{1}=z_{0}+u_{z} \cdot s
\end{aligned}
$$

\subsubsection{Absorption}

The weight of the photon, $w_{i}$, is reduced due to absorption of the media according to

$$
w_{i}=w_{i-1}\left(1-\frac{\mu_{a}}{\mu_{t}}\right)
$$

Since it is time consuming to track photons with very small weights until they are detected or cross the boundaries of the simulated media, a threshold value for the photon weight is usually implemented to reduce computational time,. The technique is called Russian roulette. When the photon weight is below this threshold value, the photon has one chance out of $m$ to survive. A random number $\xi$ is generated and if $\xi>1 / m$, the photon is terminated by setting $w=0$. Else if $\xi \leq 1 / m$, the photon weight is increased by $w_{i}=m w_{i-1}$, due to conservation of energy, and the photon continues to propagate.

It is also possible not to use weights in the simulation and instead, in each step, randomly sample survival or termination of the photon. 


\subsubsection{Scattering}

The direction of the photon will be determined based on the phase function. The deflection angle $\theta$ is dependent on the phase function and the azimuthal angle $\psi$ is randomly chosen, uniformly distributed between 0 and $2 \pi$ :

$$
\psi=2 \pi \xi
$$

The deflection angle can be calculated for a Henyey-Greenstein phase function as a function of a random number $\xi$ according to

$$
\cos \theta=\frac{1}{2 g}\left(1+g^{2}-\left(\frac{1-g^{2}}{1-g+2 g \xi}\right)^{2}\right) .
$$

The new photon direction can then be calculated as

$$
\begin{aligned}
& u_{x}^{\prime}=\frac{\sin \theta}{\sqrt{1-u_{z}^{2}}}\left(u_{x} u_{z} \cos \psi-u_{y} \sin \psi\right)+u_{x} \cos \theta \\
& u_{y}^{\prime}=\frac{\sin \theta}{\sqrt{1-u_{z}^{2}}}\left(u_{y} u_{z} \cos \psi-u_{x} \sin \psi\right)+u_{y} \cos \theta \\
& u_{z}^{\prime}=-\sin \theta \cos \psi \sqrt{1-u_{z}^{2}}+u_{z} \cos \theta
\end{aligned}
$$

and the new position as

$$
\begin{aligned}
& x_{i+1}=x_{i}+u_{x}^{\prime} \cdot s \\
& y_{i+1}=y_{i}+u_{y}^{\prime} \cdot s . \\
& z_{i+1}=z_{i}+u_{z}^{\prime} \cdot s
\end{aligned}
$$

\subsubsection{Doppler shift}

Scattering by a moving particle will cause a Doppler shift, described in section 5.1. In Monte Carlo simulations, the calculated Doppler shift is stored for each photon as an extra property. The scattering vector is calculated from

$$
\mathbf{q}=\frac{n}{\lambda}\left(\mathbf{u}-\mathbf{u}^{\prime}\right)
$$

where $n$ is the refractive index of the media, $\lambda$ is the laser wavelength, $\mathbf{u}$ is the direction vector of the incoming photon and $\mathbf{u}^{\prime}$ is direction vector of the scattered photon. The velocity $v$ is chosen randomly from a speed distribution and $\cos \varphi$ is chosen from a uniform distribution between -1 and 1. The total Doppler shift for the photon in the media is then the sum of all individual shifts. In this 
way it is possible to simulate Doppler shift even though the Doppler effect is a wave phenomenon and Monte Carlo is based on particles.

\subsubsection{Detection}

At the border, the photon can be reflected or transmitted. This is decided by using Fresnel's formula:

$$
R\left(\theta_{i}\right)=\frac{1}{2}\left(\frac{\tan ^{2}\left(\theta_{i}-\theta_{t}\right)}{\tan ^{2}\left(\theta_{i}+\theta_{t}\right)}+\frac{\sin ^{2}\left(\theta_{i}-\theta_{t}\right)}{\sin ^{2}\left(\theta_{i}+\theta_{t}\right)}\right)
$$

The reflection coefficient $R\left(\theta_{i}\right)$ is dependent on the angle of the incident light $\theta_{i}$ and on the angle of transmission $\theta_{t}$, given by Snell's law

$$
n_{i} \sin \theta_{i}=n_{t} \sin \theta_{t}
$$

If $R\left(\theta_{i}\right)<\xi$, the photon is transmitted. If the transmission occurs in a predefined area, the photon is detected.

\subsection{PoST PROCESSING}

It is also possible to add the effect of absorption and scattering in the post processing, called white Monte Carlo [81]. Each photon detection position and path length is then stored and rescaled in the post processing to describe a medium with different optical properties than the simulated. The initial absorption and scattering coefficient setup for the simulation is here called $\mu_{a, i n i}$ and $\mu_{s, i n i}$.

To add scattering effects in the post processing, each photon position and step length in the media can be rescaled with $\alpha=\mu_{s, \text { ini }} / \mu_{s, \text { new }}$.When rescaling the step length for a different scattering coefficient, the path length will also scale when adding the absorption in the post processing $d^{\prime}=\alpha d$. Absorption effects are then added by modifying the weight of each photon by the Beer-Lambert law,

$$
w^{\prime}=w \exp \left(-\left(\mu_{a, \text { new }}-\mu_{a, \text { ini }}\right) d^{\prime}\right) \text {. }
$$

Scaling the scattering coefficient also affects all other geometries in the model like source-detector distances and layer structures. 



\section{LIGHT TRANSPORT MODELS FOR DRS AND LDF}

A model of the tissue is required to quantify the content of chromophores and perfusion in tissue. Based on the tissue model, simulated spectra can be generated and compared to measured spectra to find optimal model parameters. The tissue model should be both physiologically and optically accurate, and there is usually a need for different models depending on tissue type. Homogenous and layered models suits different tissue structures, such as the brain and the skin respectively. Predefined chromophores with wavelength dependency can be used to model the absorption coefficient of tissue, and the fraction of each chromophore is estimated from the model fitting [13, 53, 92]. Another approach is to estimate the absorption coefficient for each wavelength and in the post processing fit chromophore spectra to the absorption coefficient [93]. In both cases, the choice of which chromophores to include needs to be adapted to the specific tissue. When a tissue model is assigned including the model parameters, a forward spectrum can be generated based on Monte Carlo simulations.

\subsection{SCATTERING MODELS}

The reduced scattering coefficient decays with wavelength and can be modeled according to [39]

$$
\mu_{s}^{\prime}(\lambda)=\alpha \cdot\left(\lambda / \lambda_{0}\right)^{-\beta},
$$

where the wavelength is normalized by $\lambda_{0}$ and $\alpha$ is the reduced scattering coefficient of tissue at $\lambda_{0}$. With this model, $\alpha$ will directly scale $\mu_{s}^{\prime}(\lambda)$ and $\beta(>0)$ will describe its decay with wavelength. Different tissue types will yield different combinations of $\alpha$ and $\beta$ [39]. For small particle distributions compared to the wavelength of the incident light, $\beta$ approaches 4 (Rayleigh scattering), while for larger particle sizes $\beta$ approaches 0.37 [94, 95]. The reduced scattering coefficient was modeled according to equation (8.1) in paper I and II.

Since $\beta$ in equation (8.1) differs for small and large particles another way to model scattering has been proposed to better describe a distribution of scattering sizes [39]. The reduced scattering coefficient is then modeled with separate scattering contributions for Mie scattering and Rayleigh scattering according to 


$$
\mu_{s}^{\prime}(\lambda)=\alpha\left[(1-\gamma)\left(\lambda / \lambda_{0}\right)^{-\beta}+\gamma\left(\lambda / \lambda_{0}\right)^{-4}\right]
$$

In equation (8.2), $\alpha=\mu_{s}^{\prime}\left(\lambda_{0}\right)$ while $\beta$ describes the wavelength dependency for the larger particles (the Mie scattering part). The fraction of Rayleigh scattering is described by $\gamma$ and $1-\gamma$ is hence the fraction of Mie scattering. The scattering coefficient was modeled according to equation (8.2) in papers III-V.

\subsection{ABSORPTION MODELS}

The total absorption coefficient of the tissue is usually modeled as a linear combination of the fraction of the absorber $n, f_{n}$ multiplied by the absorption coefficient for that absorber $\mu_{a, n}$ :

$$
\mu_{a}(\lambda)=\sum_{n} f_{n} \mu_{a, n}(\lambda)
$$

The included absorbers in equation (8.3) can vary depending on the tissue or the phantom under investigation and also on the wavelength range of interest. For example there is no melanin present in the heart while this is a large contributor to skin tissue absorption. When modeling the skin in the visible wavelength range, commonly included chromophores are melanin and hemoglobin with a strong absorption in this region. Water, myoglobin, methemoglobin, bilirubin, fat and cytochromes are examples of other absorbers that can be included in various tissue types [39]. The choice of which chromophores to include is important since a missing chromophore can affect the ability of the model to mimic measured spectra [96] [I]. However, as the number of chromophores in the model increases the number of free fitting parameters also increases.

\subsubsection{Melanin}

The melanin content in the epidermis varies and also the relationship between the melanosomes eumelanin and pheomelanin, making it difficult to theoretically derive an expression for cutaneous melanin. On average, the total absorption spectrum of cutaneous melanin can be approximated by the expression from Jacques et al. [53], where $\beta_{m e l}$ can be fixed or allowed to vary

$$
\mu_{a, m e l}(\lambda)=6.6 \cdot 10^{10} \lambda^{-\beta_{m e l}}
$$

The value of $\beta_{m e l}$ has been approximated to being 3 [39], but it differs for eumelanin and pheomelanin (2.45 and 3.81, respectively [39]). By having a free $\beta_{m e l}$, the model can better handle different distributions of eumelanin and pheomelanin and hence adapt to individual differences. A variable exponent was implemented in papers III-V ( $\beta_{m e l}=3.48$ in paper I). 


\subsubsection{Blood}

The absorption coefficient of blood depends on the hemoglobin concentration in blood $\left(c_{H b, \text { blood }} \approx 150 \mathrm{~g} / \mathrm{l}\right)$, the absorption coefficient for oxygenized and deoxygenized hemoglobin and also on the oxygen saturation $s=f_{\mathrm{HbO}_{2}} /\left(f_{\mathrm{Hb}}+f_{\mathrm{HbO}_{2}}\right)$ :

$$
\mu_{a, b l o o d}(\lambda)=c_{H b, b l o o d}\left(s \mu_{a, H b O_{2}}(\lambda)+(1-s) \mu_{a, H b}(\lambda)\right) \text {. }
$$

The relationship between fraction of blood and fraction of $\mathrm{RBC}$ is dependent on the hematocrit (the volume percentage of $\mathrm{RBC}$ in blood):

$$
f_{\text {blood }}=\frac{f_{R B C}}{\text { hematocrit }}
$$

Normal values of hematocrit are $38-46 \%$ for women and $40-54 \%$ for men [19]. A hematocrit of $43 \%$ was used in paper III-V.

\subsection{MODELING INHOMOGENEOUSLY DISTRIBUTED BLOOD}

Chromophores or scattering particles can be considered homogenously distributed in the tissue model, or in a layer in the model. However, the chromophore hemoglobin in real tissue is found in blood vessels which is only a small part of the tissue layer. Assuming a homogenous distribution of hemoglobin in tissue, the model will overestimate the effect of blood on the absorption coefficient leading to erroneous estimations of other parameters e.g. oxygen saturation and RBC tissue fraction [97-99] [I]. It is therefore important to compensate for inhomogeneously distributed blood in the model. This is called the vessel packaging effect and will affect the modeling of the absorption coefficient.

With correction for vessel packaging, modeled DRS spectrum will be flattened and the wavelength band where hemoglobin has characteristic absorption peaks (542 and $577 \mathrm{~nm}$ ) will be less pronounced, see Figure 8.1 [100, 101]. A flattening of the DRS spectrum, will lead to a reduced estimated fraction of blood as compared to when compensating for vessel packaging. For LDF, the vessel packaging effect will change the shift distribution mainly because consecutive shift will be caused by RBCs with similar speed. The vessel packaging compensation for LDF is described in section 8.4.4. 

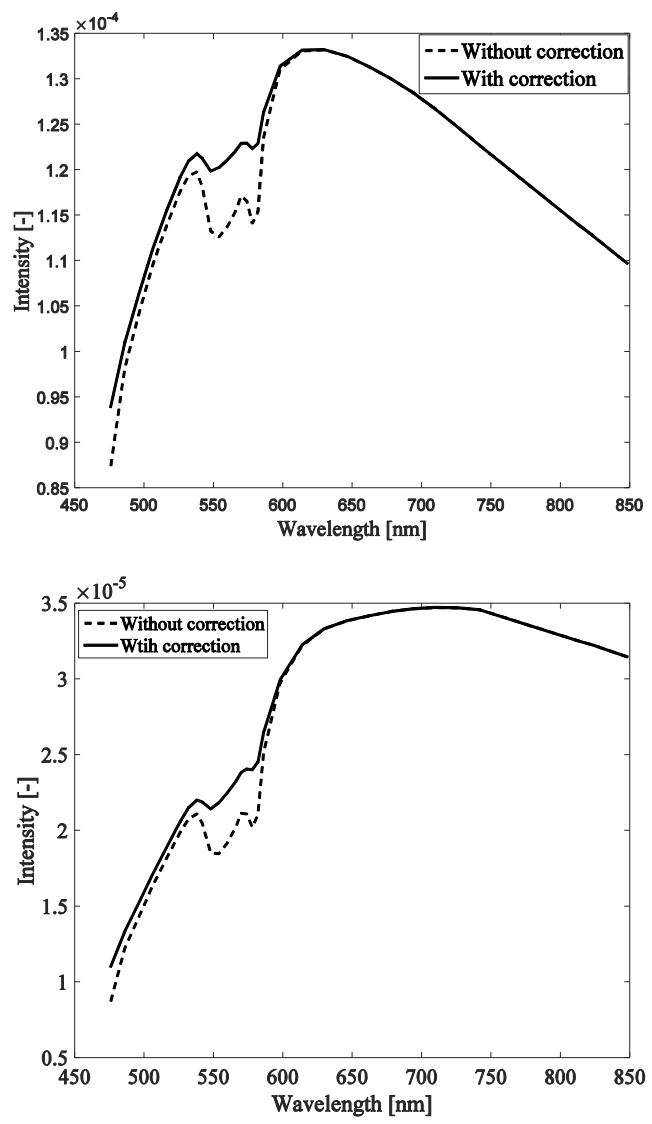

Figure 8.1. Simulated spectra at $0.4 \mathrm{~mm}$ (top panel) and $1.2 \mathrm{~mm}$ (bottom panel) source detector distance, without correction for inhomogeneously distributed blood (dashed) and with correction (solid)..

Different correction factors for DRS have been proposed [97, 101-103] that are derived analytically or with Monte Carlo simulations. The wavelength dependent correction factor, $C$ [-], for inhomogeneously distributed blood in the light transport model used in paper I and III-IV was proposed by Svaasand et al. [103] and depends on the vessel radius, $R$, and the absorption coefficient of blood $\mu_{a, b l o o d}$ :

$$
C(\lambda)=\frac{1-\exp \left(-2 R \mu_{a, b l o o d}(\lambda)\right)}{2 R \mu_{a, b l o o d}(\lambda)}
$$

The absorption coefficient with correction for inhomogeneously distributed blood is then calculated as

$$
\mu_{a}(\lambda)=C(\lambda) \cdot f_{\text {blood }} \mu_{a, \text { blood }}(\lambda)
$$


Since the correction factor is both dependent on the average vessel radius and on the wavelength dependent absorption coefficient of blood, it will have a large influence on the estimated absorption coefficient at wavelengths with high absorption of blood. Van Veen et al. [101] estimated the error between a homogeneous and an inhomogeneous model to be largest for wavelengths below $600 \mathrm{~nm}$ where blood has strong absorption. At longer wavelengths $(>600 \mathrm{~nm})$, where absorption of blood is low, the correction factor will be close to one and, hence, the effect on DRS spectra from vessel packaging will be minimal, see Figure 8.2.

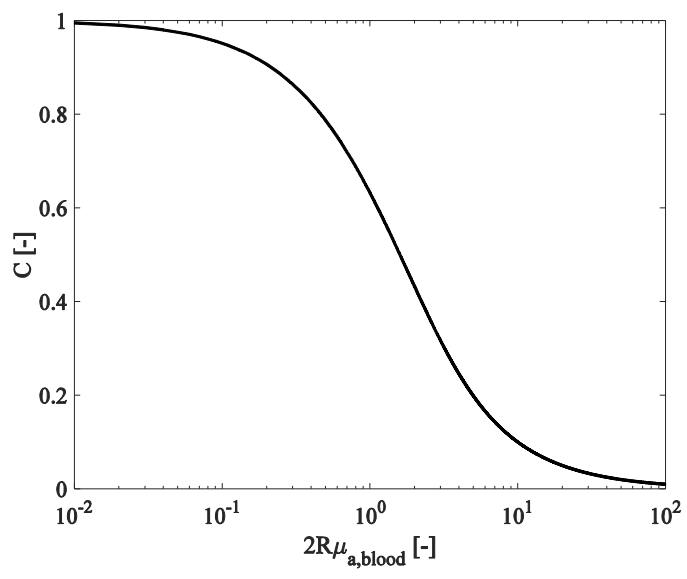

Figure 8.2.The vessel packaging correction factor as a function of the product vessel radius and the absorption coefficient of blood.

\subsection{FORWARD DRS AND LDF}

The principle of forward DRS and LDF is to calculate model spectra from the skin model based on Monte Carlo simulations. A flowchart for the forward problem in shown in Figure 8.3. In this thesis, pre-simulated spectra with various scattering coefficients and epidermal thicknesses were used. Based on the model parameters, linear interpolation was performed among the simulated scattering coefficients and epidermal thicknesses for each wavelength to create modeled DRS and LDF spectra (only one wavelength). The effect of absorption was added by the Beer-Lambert law and compensation for vessel packaging was performed. 


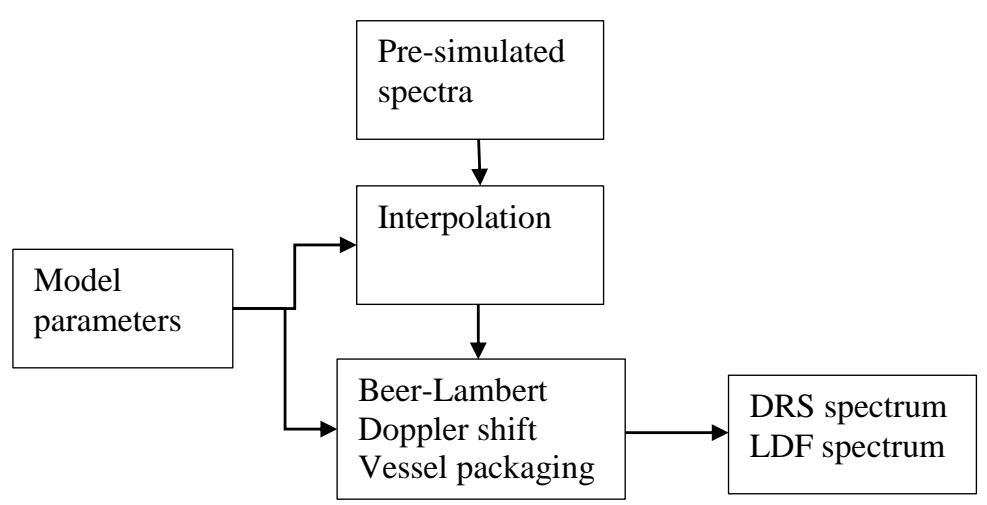

Figure 8.3. Flowchart of the forward problem for DRS and LDF.

\subsubsection{Three layer skin model}

The skin was modeled as a three-layer structure in paper III-V according to Fredriksson et al. [104]. The model consisted of one bloodless (epidermis) layer with a variable thickness and two dermal layers, where the upper layer had a fixed thickness $(0.5 \mathrm{~mm}$ paper III and IV, $0.2 \mathrm{~mm}$ paper V) and the lower an infinite thickness, see Figure 8.4. The two dermis layers were allowed to have different fractions of blood ( $f_{\text {blood }, 1}$ and $f_{\text {blood }, 2}$, respectively), calculated from an average fraction and a relative ratio between the layers. The blood was assumed to be inhomogeneously distributed and confined to vessels with an average radius, $R$. The oxygen saturation was allowed to be different in the two dermis layers in Paper V, while in paper III and IV it was assumed to be equal for the two layers $\left(\mu_{a, \text { blood }, 1}=\mu_{a, \text { blood }, 2}\right)$. 


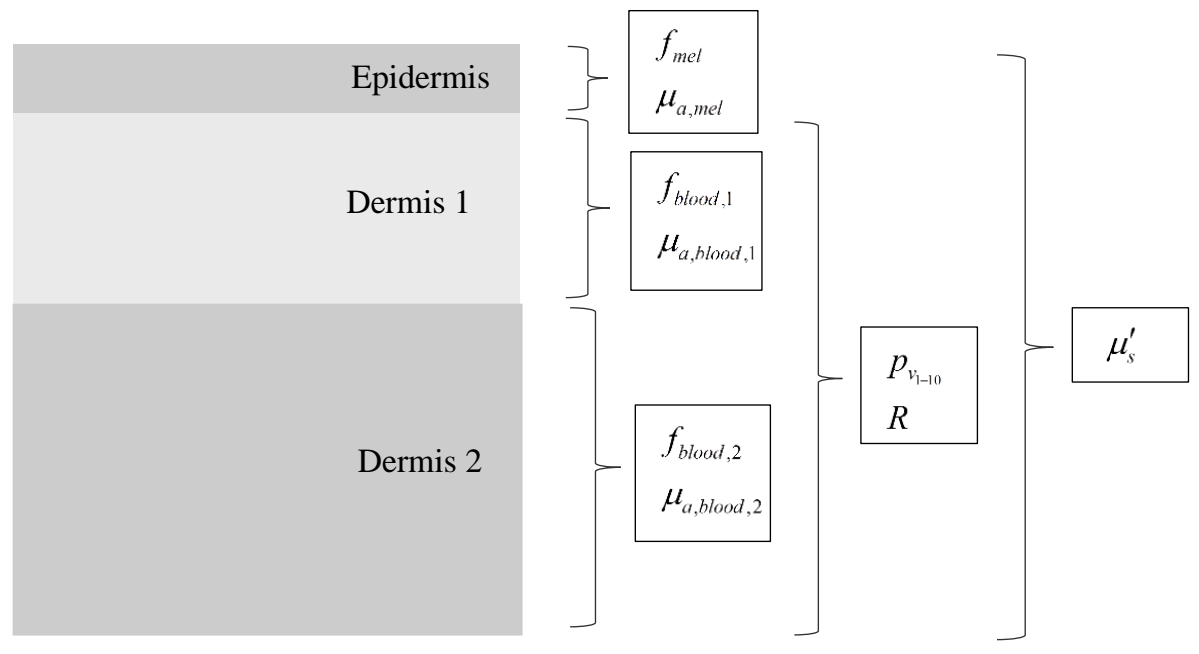

Figure 8.4. Skin model with model parameters

A speed distribution with ten parameters $p_{v_{1-10}}$ described the blood flow in the tissue. Each of the parameters consisted of a rectangular distribution of speeds between 0 and $2 v$ where $v$ is the average speed of each parameter, chosen between 0 and $75 \mathrm{~mm} / \mathrm{s}$, Figure 8.5. The rectangular distribution mimicked the flow found in vessels with a laminar flow.

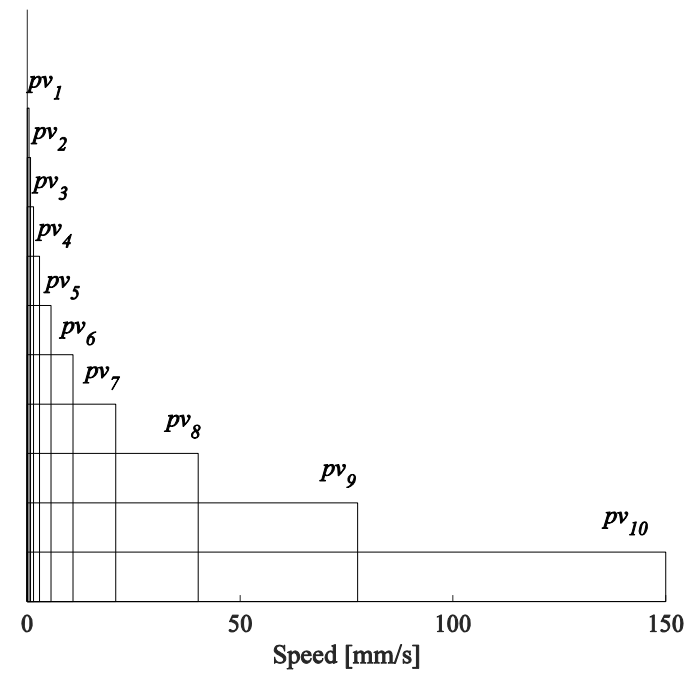

Figure 8.5. Speed distribution 


\subsubsection{Path length distributions}

Simulated photons can be expressed in path length distributions, $p_{p l}(d)$ that describes the probability of detected intensity for a given path length $d$. These simulated path length distributions are then interpolated based on the scattering coefficient and epidermal thickness of the model. In a homogenous one-layer medium, the total probability of detected intensity is $I=\sum p_{p l}(d)$ and the effect of absorption can be added by modify the expression with the Beer-Lambert law

$$
I=\sum p_{p l}(d) \exp \left(-d \mu_{a}\right)
$$

For a multi-layer medium with layer number $m=1,2,3$, the photons are grouped together by the deepest layer they have reached, $n=1,2,3$. This generates six path length distributions $p_{p l, m, n}(d)$ for the layers (three path length distributions for layer 1, two for layer 2 and one for layer 3), Figure 8.6.

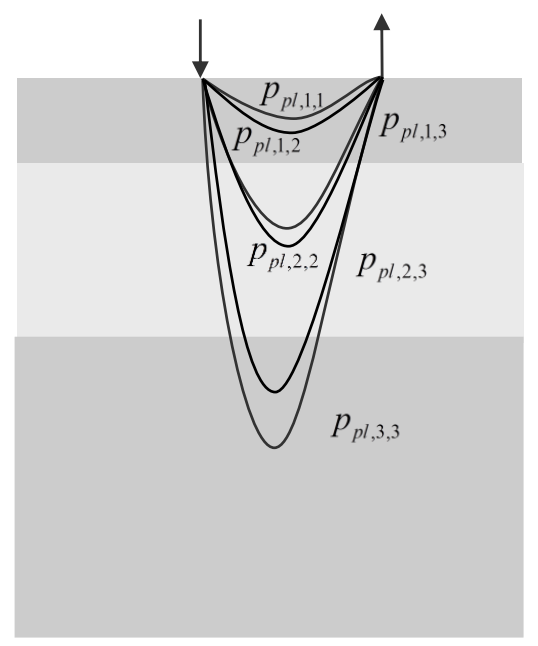

Figure 8.6. Path length distributions

\subsubsection{Forward DRS}

To calculate a forward DRS spectrum from a three layer model, the effect of absorption from each layer on the total detected intensity needs to be calculated. The detected intensity, $I_{0, n}$ from photons that had reached each layer $n$ is calculated as

$$
I_{0, n}=\sum_{d} p_{p l, m, n}(d)
$$


The path length distributions are then normalized by $I_{0, n}$, i.e. $\sum p_{p l, m, n}(d)=1$. For a multi-layer medium, it is necessary to normalize before applying the Beer-Lambert law since all layers can affect the detected intensity differently. The path length distributions are then modified by the Beer-Lambert law

$$
p_{p l, m, n}^{\prime}(d)=p_{p l, m, n}(d) \exp \left(-d \mu_{a, m}\right)
$$

where $\mu_{a, m}$ is the absorption for layer $m$. By assuming the path lengths in each layer are independent of the other layers, the effect of absorption for photons that reached layer $n$ can be calculated by multiply the effect of each layer the photons have propagate in:

$$
I_{n}=\prod_{m=1}^{n} \sum_{d} p_{p l, m, n}^{\prime}(d)
$$

The probability of detected intensity for photons that reached layer $n$ is then calculated by multiply equation (8.12) with the normalization factor $I_{n}^{\prime}=I_{0, n} I_{n}$. Finally, the total probability of detected intensity for all photons can be calculated as $I=I_{1}^{\prime}+I_{2}^{\prime}+I_{3}^{\prime}$.

\subsubsection{Forward LDF}

The forward LDF spectra calculations starts by following the steps described in previous section 8.4.3. Based on the speed distribution, a single-shifted optical Doppler spectrum $I_{1, v}(f)$ can be calculated analytic described by Fredriksson et al. [99]. The optical Doppler spectrum for any number of shifts $s$ is then calculated by cross-correlation as

$$
I_{s, v}(f)=I_{s-1, v}(f) \bullet I_{1, v}(f) .
$$

By assuming symmetry of the optical Doppler spectra, the cross-correlation is equal to a convolution. By Fourier transforming the optical Doppler spectrum $\mathcal{F}\left\{I_{1, v}(f)\right\}=Y_{v}(\gamma)$, the convolution corresponds to a multiplication $\mathcal{F}\left\{I_{s, v}(f)\right\}=Y_{v}^{s}(\gamma)$.

The vessel packaging compensation for LDF is performed by calculated the optical Doppler spectrum from a single vessel and summarize over a distribution of vessels. In a single vessel, multiple shift can be described by a Poisson distribution with an expectation value $\hat{n}_{\text {shift }}$ based on the vessel radius $R$ and the scattering coefficient of blood $\mu_{s, \text { blood }}, \hat{n}_{\text {shift }}=2 R \mu_{s, \text { blood }}$. The Fourier transformed optical Doppler spectrum can then be altered to account for multiple shifts in a single vessel, according to Fredriksson et al. [99]: 


$$
Y_{v e s s e l, v}(\gamma)=\sum_{s} Y_{v}^{s}(\gamma) p_{P o}\left(s ; \hat{n}_{\text {shift }}\right)
$$

where $p_{P o}\left(s ; \hat{n}_{\text {shift }}\right)$ is the probability of $s$ shifts based on a Poisson distribution with parameter $\hat{n}_{\text {shift }}$. These multiple shifted spectra are then summarized over the distribution of vessels with various speeds

$$
Y_{\text {vessel }}(\gamma)=\sum_{j} f_{\text {blood, } v_{j}} Y_{\text {vessel, } v_{j}}(\gamma)
$$

where $f_{\text {blood, } v_{j}}$ is the fraction of blood moving with speed $v_{j}$.

The number of passed vessels in a layer $m$ can be described by a Poisson distribution with expectation value $\hat{n}_{\text {vessel }}=d \cdot f_{\text {blood, }, m} /(2 R)$ according to Fredriksson et al [99]. The probability of $k$ passed vessels for a Poisson distribution with parameter $\hat{n}_{v e s s e l}$ is given by $p_{P_{o}}\left(k ; \hat{n}_{\text {vessel }}\right)$. A distribution of passed vessels is then calculated from the normalized path length distribution $p_{p l, m, n}^{\prime \prime}(d)=p_{p l, m, n}^{\prime}(d) / \sum p_{p l, m, n}^{\prime}(d)$, modified for absorption (section 8.4.3), by

$$
p_{v e s s e l, m, n}(k)=\sum_{d} p_{p l, m, n}^{\prime \prime}(d) p_{P o}\left(k ; \hat{n}_{v e s s e l}\right)
$$

Then, for photons in each layer $m$ that have been propagating down to layer $n$, a Fourier transformed optical Doppler spectrum for a number of passed vessels is calculated as

$$
Y_{m u l t . v e s s e l s, m, n}(\gamma)=\sum_{k=0}^{K} p_{\text {vessel }, m, n}(k) Y_{\text {vessel }, m, n}(\gamma)
$$

where $K$ is the maximum number of passed vessels.

The Fourier transformed optical Doppler spectrum including multiple shifts and vessel packaging for all layers can then be calculated as

$$
Y_{\text {tot }}(\gamma)=\sum_{n=2}^{3} I_{m}^{\prime} \prod_{m=2}^{n} Y_{m u l t . v e s s e l s, m, n}(\gamma)
$$

where $I_{m}^{\prime}=I_{m} / \sum_{m} I_{m}$. Finally, the Doppler power spectrum is calculated by

$$
P(f)=\mathcal{F}^{-1}\left\{Y_{\text {tot }}^{2}(\gamma)\right\} .
$$




\section{INVERSE MODELING OF DRS AND LDF}

Measured DRS and LDF spectra carry information about the optical properties of tissue. With a skin model these optical properties can be assigned to different aspects of the microcirculation, such as chromophore content, oxygen saturation and speed of the blood flow. To find model parameters that best describes measured DRS and LDF spectra is called the inverse problem. Based on the skin model and Monte Carlo simulations, the goal of the inverse modeling is to find a set of parameters minimizing the difference between measured and simulated spectra. In order to estimate the parameters in real time, solutions to decrease the fitting time is important. With an integrated system for DRS and LDF, this set of parameters can then describe the microcirculatory blood flow, fraction of red blood cells and the oxygen saturation in the skin simultaneously and in absolute units.

\subsection{MEASUREMENT SYSTEM}

The measurements in paper I and III-V were performed using a custom made fiber optic probe consisting of two emitting fibers near the center of the probe tip and nine detecting fibers, see Figure 9.1. One emitting fiber was connected to a broadband white light source (Avalight-HAL-S, Avantes BV, The Netherlands) (Paper I, III-V) and one emitting fiber was connected to a laser light source $(780 \mathrm{~nm})$ (Paper III-V), see Table 9.1. Two of the detecting fibers collected DRS light and were positioned at a source-detector distance of 0.4 and $1.2 \mathrm{~mm}$ and the remaining seven detecting fibers collected LDF light at a distance of 0.4 (one fiber) and $1.2 \mathrm{~mm}$ (six fibers). The LDF detector fibers were connected to one detector for each distance (the six $1.2 \mathrm{~mm}$ fibers joining into one) in a modified Periflux 5000 (Perimed AB, Järfälla, Sweden). In paper II a fiber optic probe with one emitting fiber, connected to the white light source, and four detecting fibers at distances of $0.22,0.46,0.68$ and $1.13 \mathrm{~mm}$ was used, see Figure 9.1.

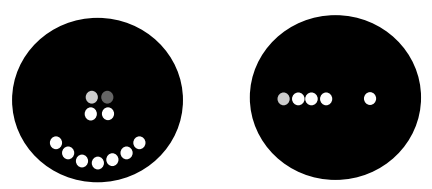

Figure 9.1. The fiber optic probes, used in paper I and III-V (left) and paper II (right). White light source (light grey), laser light source (dark grey), detectors (white). 
In paper I-IV the detecting fibers for diffuse reflectance spectra were connected to a multi-channel spectrometer (AvaSpec 2048-5-RM, Avantes BV, the Netherlands). In paper I the intensity of the lamp was also logged by one separate spectroscopic channel. In paper $\mathrm{V}$, the two detector fibers were connected to one spectrometer each (AvaSpec-ULS2048L, Avantes BV, The Netherlands). An optical notch filter was added on the spectrometer inputs to suppress wavelengths at $790 \pm 20 \mathrm{~nm}$ to achieve minimal influence of laser light to DRS spectra. All fibers had a diameter of $200 \mu \mathrm{m}$ and a numerical aperture of 0.37 .

Table 9.1. Summary of source-detector distances for DRS and LDF and spectrometers used in papers $I-V$.

\begin{tabular}{|c|c|c|c|}
\hline & Probe distance DRS & Probe distance LDF & Spectrometer \\
\hline Paper I & $0.4,1.2$ & - & Multi-channel \\
\hline Paper II & $0.22,0.46,0.68,1.13$ & - & Multi-channel \\
\hline Paper III & $0.4,1.2$ & 0.4 & Multi-channel \\
\hline Paper IV & $0.4,1.2$ & 1.2 & Multi-channel \\
\hline Paper V & $0.4,1.2$ & 1.2 & Two single-channel \\
\hline
\end{tabular}

\subsection{DRS CALIBRATION}

To be able to get accurate output parameters, all other factors influencing measured spectra need to be handled. Calibration of measured spectra compensates for factors such as system noise, the color and intensity of the light source, the numerical aperture of the fibers and the spectrometer sensitivity.

When fitting MC simulated spectra to measured spectra, the average intensity difference between measured and simulated spectra needs to be handled either in the calibration or in the fitting procedure. Intensity calibration with a calibration phantom used as reference object is in this thesis referred to as absolute calibration. In the absolute calibration method, simulated phantom spectra with known optical properties are compared to measured phantom spectra in order to gain one or multiple absolute calibration factors. Relative calibration factors for the individual fibers can be generated with a uniform illumination of multiple detector distances where the detected intensity in the fibers are normalized. This allows for intensity comparison between source detector distances while the overall intensity difference between modeled and measured spectrum is removed in the fitting procedure.

The full calibration procedure is described in the following subchapters.

\subsubsection{Noise correction}

A measurement with the light source turned off must be used to remove system noise. By simply subtracting the dark spectrum $I_{\text {dark }}(\rho, \lambda)$ (dependent of the fiber separation $\rho$ and the wavelength) 
from the measured spectrum $I_{\text {meas }}(\rho, \lambda)$, noise-correction of spectrum is performed to obtain $I_{\mathrm{d} \text {,meas }}(\rho, \lambda)$ :

$$
I_{\mathrm{d} \text {,meas }}(\rho, \lambda)=I_{\text {meas }}(\rho, \lambda)-I_{\text {dark }}(\rho, \lambda)
$$

\subsubsection{Color correction}

Coloring of measured spectra introduced by the optical fiber, the spectrometer and the light source that are not originating from the chromophores in the tissue can be corrected/compensated for by a using a white reference. In this thesis, a surface covered with barium sulphate (WS-2 white reference tile, Avantes) was used as white reference with approximately $98 \%$ reflectivity. A reflection spectrum, $I_{\mathrm{d}, \mathrm{BaSO}_{4}}(\rho, \lambda)$, from this surface in the visible wavelength range was used to normalize the measured spectrum. The normalization is described by

$$
I_{\mathrm{w}, \mathrm{d}, \text { meas }}(\rho, \lambda)=\frac{I_{\mathrm{d}, \text { meas }}(\rho, \lambda)}{I_{\mathrm{d}, \mathrm{BaSO}_{4}}(\rho, \lambda)}
$$

\subsubsection{Post processing color correction}

An intensity/color reference can be used in situations where it is not possible to perform a white reference measurement. Since the intensity reference then is the link between measurement occasions, factors such as consistent optical properties of the intensity reference is important. Also, the probe position relative the intensity reference need to be consistent and repeatable. At the measurement occasion, a measurement on the intensity reference is performed and the intensity reference measurement is repeated at a separate occasion, then together with a white reference measurement. A measured spectrum can then be calibrated as

$$
I_{\mathrm{w}, \mathrm{d}, \text { meas }}(\rho, \lambda)=\underbrace{\frac{I_{\mathrm{d}, \text { meas }}(\rho, \lambda)}{I_{\mathrm{d}, \mathrm{i}, \mathrm{r}, 1}(\rho, \lambda)}}_{\text {occasion } 1} \cdot \underbrace{\frac{I_{\mathrm{d}, \mathrm{i} . \mathrm{r}, 2}(\rho, \lambda)}{I_{\mathrm{d}, \mathrm{BaSO}{ }_{4}}(\rho, \lambda)}}_{\text {occasion 2 }}
$$

System drift during a measurement occasion can be assessed if multiple measurements on the intensity reference is performed, for example before and after the measurement occasion. If there is a color or intensity difference between such before and after measurements, the system drift has probably affected measured spectra. Since the system drift cannot be assumed to be linear, the effect on measured spectra is unknown and is therefore difficult to compensate for. In such cases the safest way is to discard measured spectra. 


\subsubsection{Absolute calibration}

Absolute calibration can be performed by using an optical phantom with known optical properties. With a polystyrene microsphere solution it is possible to calculate the phase function with Mie theory, since the refractive index of the solution and the size of the particles is known. Together with a spectral collimated transmission (SCT) measurement to determine the scattering coefficient, the scattering properties of the microsphere solution can be described. Spectra for the microsphere solution can then be simulated and compared to measured spectra:

$$
A_{\text {abs }}(\rho)=\left\langle\frac{I_{\text {microsphere }}(\lambda, \rho)}{I_{\text {MC,microsphere }}(\lambda, \rho)}\right\rangle_{\lambda} .
$$

The calculated ratio between simulated and measured spectra is used as calibration factors

$$
I_{\mathrm{w}, \mathrm{d}, \mathrm{abs}}(\lambda, \rho)=\frac{I_{\mathrm{w}, \mathrm{d}, \mathrm{meas}}(\lambda, \rho)}{A_{\mathrm{abs}}(\rho)}
$$

The intensity of the absolute calibrated spectra are directly comparable to simulated spectra without further calibration.

\subsubsection{Relative calibration}

If no absolute calibration is performed, multiple detector distances are needed to assess optical properties. When the detector distances are relative calibrated against each other, there is no need for a reference phantom which can be seen as an advantage as the absolute calibration procedure includes many steps where errors can be introduced.

The calibration factors are calculated from a measurement where all detector fibers are uniformly illuminated, $I_{\text {u.i. }}(\lambda, \rho)$. The uniform illumination can be achieved by using an integrated sphere or submerging the probe into, or holding the probe against, a highly scattering medium illuminated by an external light source (the probe light source is turned off), Figure 9.2.

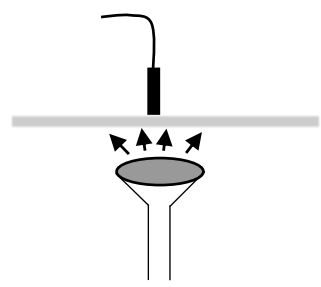

Figure 9.2. Uniform illumination of the probe by using a highly scattering medium and an external light source. 
The calibration factors are calculated as the normalized mean intensity over a wavelength range with high intensity of the backscattered light.

$$
A_{\text {rel }}(\rho)=\left\langle\frac{I_{\text {w,d,u.i. }}(\lambda, \rho)}{\left\langle I_{\text {w,d,u.i. }}(\lambda, \rho)\right\rangle_{\rho}}\right\rangle_{\lambda} .
$$

Measured calibrated spectra are then calculated as

$$
I_{\mathrm{w}, \mathrm{d}, \mathrm{rel}}(\lambda, \rho)=\frac{I_{\mathrm{w}, \mathrm{d}, \mathrm{meas}}(\lambda, \rho)}{A_{\mathrm{rel}}(\rho)}
$$

\subsection{LDF CALIBRATION}

Background light and electronic noise from the LDF device can affect LDF spectra. Repeated measurements of the Doppler power spectrum on an object without any moving scatters for different light intensity levels (dc-levels), results in an intensity dependent noise spectrum that can be subtracted from measured Doppler power spectrum. The noise corrected Doppler power spectrum is then $i_{\mathrm{dc}}^{2}$-normalized:

$$
P(f)=\frac{P_{\text {tissue }}(f)-P_{\text {noise }}(f, d c)}{i_{\mathrm{dc}}^{2}} .
$$

To be able to get perfusion values in absolute units, a calibration against a reference perfusion value is necessary. By using a scattering media such as a polystyrene microsphere solution in which Brownian motion of the scatters can provide a reference value, measured tissue perfusion values can be quantified. Calibration can be done either with the perfusion value (the first order moment of the Doppler spectrum) of the scattering media

$$
\text { Perfusion }_{\text {scatt }}=\frac{\int_{-\infty}^{\infty} f \cdot\left(P_{\text {scatt }}(f)-P_{\text {noise }}\left(f, d c_{\text {scatt }}\right)\right) d f}{i_{\mathrm{dc}, \text { scatt }}^{2}}
$$

or with the concentration of moving red blood cells (CMBC) value (the zero order moment of the Doppler spectrum) [105]

$$
C M B C_{\text {scatt }}=\frac{\int_{-\infty}^{\infty} P_{\text {scatt }}(f)-P_{\text {noise }}\left(f, d c_{\text {scatt }}\right) d f}{i_{\mathrm{dc}, \text { scatt }}^{2}}
$$


In this thesis, the noise corrected LDF spectra are normalized with the CMBC value of the reference measurement:

$$
P_{\text {calib }}(f)=\frac{P(f)}{C M B C_{\text {scatt }}} \text {. }
$$

By calibrating with $\mathrm{CMBC}$ instead of with the perfusion value, the calibration procedure is insensitive to the velocity of the moving scattering particles, to the temperature of the calibration solution and to the properties of the solution [105].

\subsection{INVERSE MODELING}

After calibration of spectra, the oxygen saturation, RBC tissue fraction and the speed resolved perfusion can be quantified by using an optimization routine, where the difference between measured and simulated spectra is minimized. The optimization is done first for DRS and secondly for LDF, and the model parameters from the DRS fitting are set prior to the LDF fitting. A flow chart of the inverse modeling can be seen in Figure 9.3.

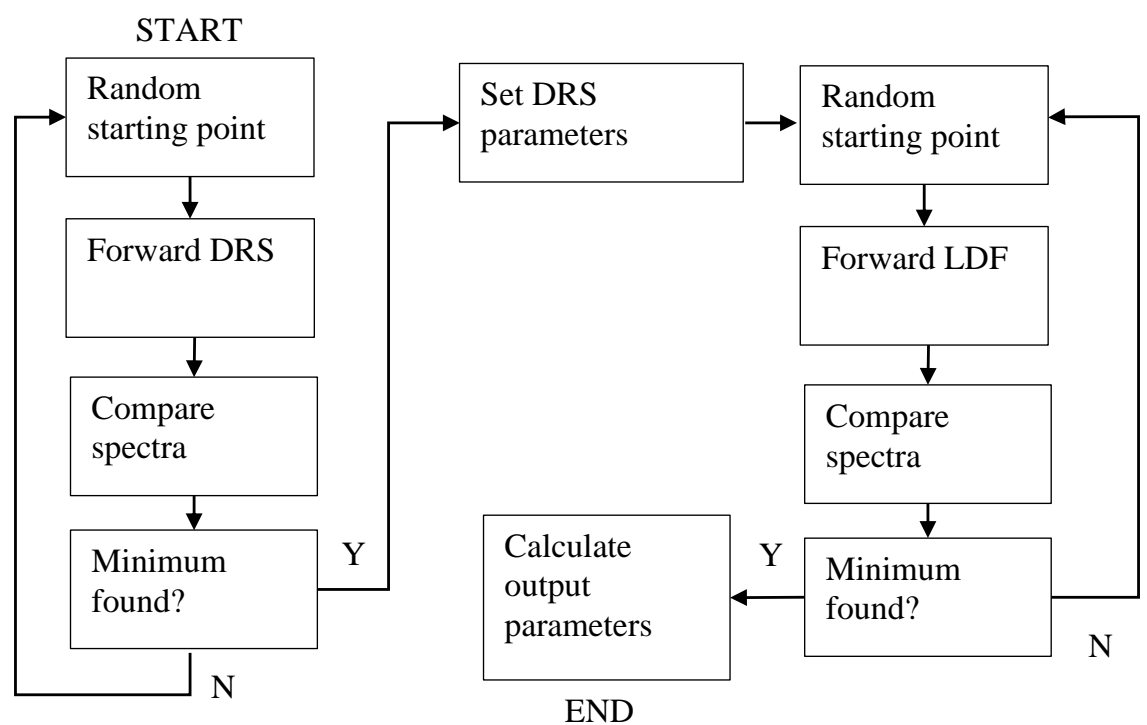

Figure 9.3. Flow chart of the inverse modeling.

The model parameters for DRS and LDF consisted of 20 parameters modeling the skin; the thickness of the bloodless layer $\left(t_{\text {epi }}\right)$, the fraction of melanin $\left(f_{\text {mel }}\right)$, the melanin power factor $\left(\beta_{\text {mel }}\right)$, the scattering $(\alpha, \beta, \gamma)$, the blood tissue fraction ( $\left.f_{\text {blood,arg }}, f_{\text {blood,rel }}\right)$, the oxygen saturation $(s)$, the mean 
vessel diameter $(R)$ and the speed distribution $\left(p_{v_{1-10}}\right)$. The first 10 parameters are derived from fitting DRS spectra and affects both DRS and LDF spectra. The second 10 parameters, i.e. the speed distribution are derived from fitting LDF spectra and affects only LDF spectra.

\subsubsection{Fitting DRS spectra}

The DRS inverse problem can be solved with a non-linear least-square optimization algorithm. When using a relative calibration method, the intensity difference between modeled and measured spectra is removed in each step of the fitting by normalizing spectra with the average intensity of both source detector distances $\rho$ :

$$
I_{m}^{\prime}(\lambda, \rho)=\frac{I_{m}(\lambda, \rho)}{\left\langle I_{m}(\lambda, \rho)\right\rangle_{\lambda, \rho}}, m \in[\text { model, meas }]
$$

The optimization algorithm minimize the objective function $F_{\mathrm{DRS}}(\mathbf{x})$ which depends on the parameters describing DRS spectra, $\mathbf{x}$ :

$$
\min _{\mathbf{x}} F_{\mathrm{DRS}}(\mathbf{x}), \text { subject to } c_{i}(\mathbf{x}) \leq 0
$$

where $\mathbf{x}=\left(t_{\text {epi }}, f_{\text {mel }}, \beta_{\text {mel }}, \alpha, \beta, \gamma, f_{\text {blood,avg }}, f_{\text {blood,rel }}, s, R\right)$ and $c_{i}(\mathbf{x}) \leq 0$ is constraints on the parameters. The parameters $\mathbf{x}$ are constrained to reflect expected or physiologically accurate estimates.

In paper I and II, the objective function included only the relative intensity difference between measured and simulated spectra, i.e. $F_{\mathrm{DRS}}=\left\|I_{\text {model }} / I_{\text {meas }}-1\right\|_{2}^{2}$. In paper III-V, the objective function also included shape properties in the hemoglobin region, deviations from unity in the intensity relaxation factor (described below) and undesirable behavior of the model parameters.

\section{Intensity relaxation factor}

Imperfections in the measurement setup or in the absolute or relative calibration factor can cause systematic deviations between fitted and measured spectra. If such deviations are not handled, the optimization routine tries to compensate for the deviation to minimize the error. In paper II the optimization algorithm compensated by introducing non-existing chromophore which led to an erroneous result. By introducing an intensity relaxation factor in the inverse fitting such misfits were handled. The intensity relaxation factor normalize measured spectra for each source detector distance in the optimization routine, $I_{\mathrm{w}, \mathrm{d}, \mathrm{abs} / \mathrm{rel}}(\lambda, \rho) \leftarrow q(\rho) \cdot I_{\mathrm{w}, \mathrm{d} \text {,abs/rel }}(\lambda, \rho)$, and is calculated in each iteration. The intensity relaxation factor $q(\rho)$ was defined for relative calibrated spectra as 


$$
q_{\mathrm{rel}}(\rho)=\left\langle\frac{I_{\text {model }}(\lambda, \rho) / I_{\mathrm{w}, \mathrm{d}, \mathrm{rel}}(\lambda, \rho)}{\left\langle I_{\text {model }}(\lambda, \rho) / I_{\mathrm{w}, \mathrm{d}, \mathrm{rel}}(\lambda, \rho)\right\rangle_{\lambda, \rho}}\right\rangle_{\lambda} .
$$

The values of the intensity relaxation factor should be limited in the optimization routine depending on measurement situation. In paper II where the phantoms and the measurement situation were controlled, $q$ could be allowed to vary $\pm 10 \%$. In the skin measurements in paper III-V, the deviations from unity in $q$ were allowed to be larger, but deviations from unity were penalized in the optimization.

\section{Chosen wavelengths}

To reduce fitting time, a subset of measured wavelengths can be selected. The selected wavelengths in paper III-V were 32 wavelengths in the 475 to $850 \mathrm{~nm}$ interval, more densely chosen in the band where hemoglobin has absorption peaks (520 to $600 \mathrm{~nm}$ ) and none around the laser wavelength (780 $\mathrm{nm}$ where optical notch filter blocked the light), see Figure 9.4.

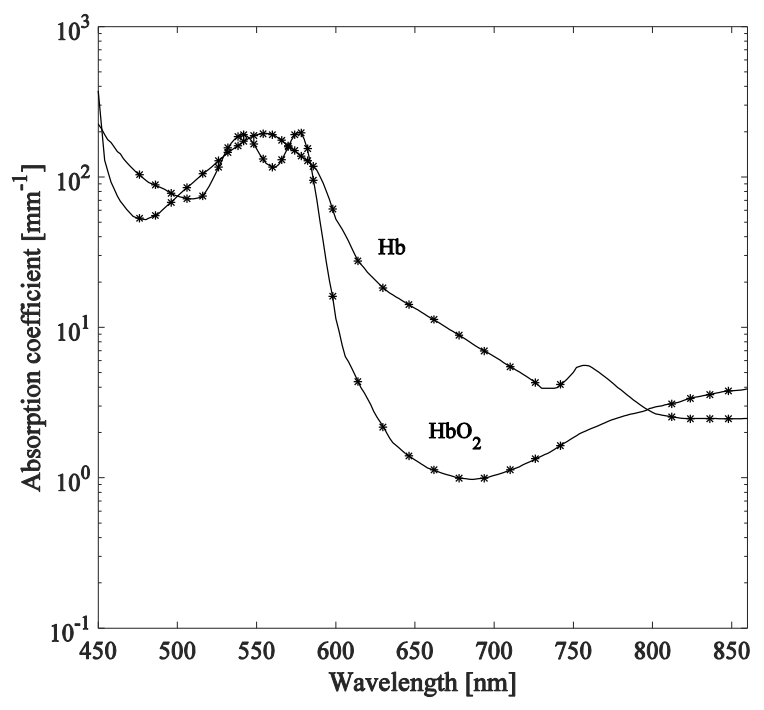

Figure 9.4. Chosen wavelengths in the optimization routine. Absorption spectra for oxygenized hemoglobin, $\mathrm{HbO}_{2}$ and deoxygenized hemoglobin, $\mathrm{Hb}$.

\subsubsection{Fitting LDF spectra}

Analogy with section 9.4.1, the LDF inverse problem can be solved with a non-linear least-square optimization algorithm: 


$$
\min _{\mathbf{p}_{v}} F_{\mathrm{LDF}}\left(\mathbf{p}_{v}\right), \text { subject to } p_{v_{1-10}} \geq 0, \sum_{j} p_{v_{j}} \leq 1
$$

The objective function for LDF, $F_{\mathrm{LDF}}\left(\mathbf{p}_{v}\right)=\left\|W\left(F_{\text {model }}\left(\mathbf{p}_{v}\right)-F_{\text {meas }}\right)\right\|_{2}^{2}$, depends on the ten speed parameters $p_{v_{1-10}}$ and $W$ is a weight matrix. In the objective function, the first order moment of the Doppler power spectrum for different frequency intervals is compared to measured

$$
F_{\text {model }}\left(\mathbf{p}_{v}\right)-F_{\text {meas }}=\int f \cdot P_{\text {model }}\left(f, \mathbf{p}_{v}\right) d f-\int f \cdot P_{\text {meas }}(f) d f \text {. }
$$

\subsection{OUTPUT PARAMETERS}

The RBC oxygen saturation, the RBC tissue fraction and the speed resolved perfusion were presented as output parameters from the system. Since the RBC tissue fraction and the perfusion, and also the oxygen saturation in paper $\mathrm{V}$ were allowed to differ between the layers, they were presented as averages over the sampling volume.

\subsubsection{Sampling volume}

The sampling volume in paper III-V was calculated as the actual volume the simulated photons had propagated in. This was done by randomly choosing at least 25000 points among the detected photon paths in the Monte Carlo simulation of the final tissue model. Figure 9.5 gives an example of a point cloud where $t_{e p i}=0.63 \mathrm{~mm}, \mu_{s}^{\prime}=8 \mathrm{~mm}^{-1}, \mu_{a}=0.2 \mathrm{~mm}^{-1}$ in the epidermis layer and $\mu_{a}=0.4 \mathrm{~mm}^{-1}$ in the dermis layers. Based on the number of included points for both detector distances and wavelengths, weighting factors for each layer are generated. The output parameters were then calculated for the whole sampling volume. 


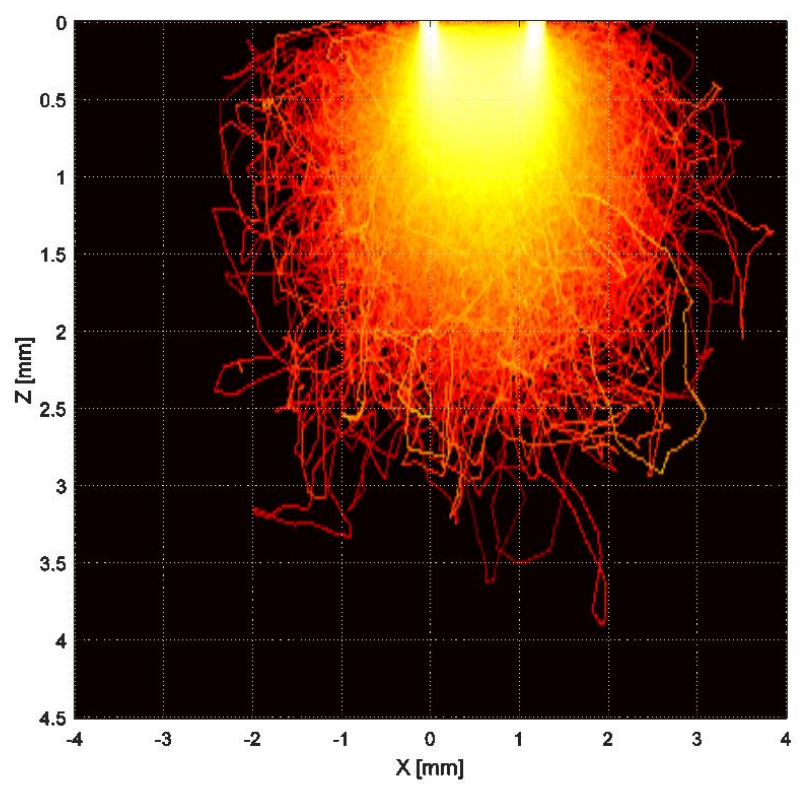

Figure 9.5. Probability point cloud. The light source is located at $(0,0)$ and the detecting fiber at (1.2,0). Both fibers have a diameter of $200 \mu \mathrm{m}$.

\subsubsection{Spatiotemporal variability}

Spatiotemporal variation in the output parameters were assessed on the forearm and the foot in paper IV. It was also assessed from the data obtained in the study in paper $\mathrm{V}$, but not presented in that paper. The coefficient of variation (CV) was calculated from two measurement sites on the forearm, and three sites on the foot. The CV was calculated from 17 subjects in study IV and 107 subjects in study $\mathrm{V}$. The variability is presented in Table 9.2.

Table 9.2. Variability expressed as coefficient of variation (CV) for the oxygen saturation, $R B C$ tissue fraction and speed resolved perfusion on the forearm and on the foot in study $I V$ and $V$.

\begin{tabular}{r|lll}
\multicolumn{5}{l}{$C V[\%]$} \\
\hline & Forearm, study IV & Foot, study IV & Foot, study V \\
\hline Oxygen saturation & 10 & 14 & 20 \\
RBC tissue fraction & 17 & 24 & 30 \\
Perfusion $0-1 \mathrm{~mm} / \mathrm{s}$ & 10 & 17 & 36 \\
Perfusion $1-10 \mathrm{~mm} / \mathrm{s}$ & 22 & 36 & 40 \\
Perfusion $>10 \mathrm{~mm} / \mathrm{s}$ & 23 & 36 & 37 \\
Total perfusion & 22 & 26 & 33
\end{tabular}




\subsection{MODEL EVALUATION}

Since the proposed model include many parameters it is important to make sure all parameters contribute significantly. Models with a reduced number of parameters can be evaluated using an $F$ test statistics, where a full model is compared to reduced models. In this test the residual sum of squares for the full model $\chi_{\text {full }}^{2}$ is compared to the residual sum of square for the reduce model $\chi_{\text {red }}^{2}$, and if the difference is significantly nonzero the reduced model can be rejected [106]. The test variable is defined as

$$
v=\frac{\chi_{\text {red }}^{2}-\chi_{\text {full }}^{2}}{n-k} / \frac{\chi_{\text {full }}^{2}}{N-n-1}
$$

where $n$ is the number of parameters in the full model, $k$ is the number of parameters in the reduced model and $N$ is the number of points in the $\chi^{2}$ statistics. The test variable $v$ has an $F$ distribution with $n-k$ and $N-n-1$ degrees of freedom. The zero hypothesis that the models are equal is rejected if $v>F(n-k, N-n-1)$ [106]. 



\section{REVIEW OF THE PAPERS}

The included papers cover tissue model development [I, II, III], accuracy of the method in the estimation of absorption and scattering in optical phantoms [II] and evaluation of calibration methods, scattering phase function and number of detectors and choice of source-detector distances [II]. Clinical evaluation of the system was performed in healthy subjects [III, IV] and in patients with diabetes [V] during microvascular reactivity tests.

\subsection{PAPER I - CAN A ONE-LAYER OPTICAL SKIN MODEL INCLUDING MELANIN AND INHOMOGENEOUSLY DISTRIBUTED BLOOD EXPLAIN SPATIALLY RESOLVED DIFFUSE REFLECTANCE SPECTRA?}

In paper I, a one layer skin model was evaluated for single and multiple source detector distances. The effect of including melanin as an absorbing chromophore into the model and compensating for inhomogeneously distributed blood (vessel packaging) were also evaluated. Measurements were performed on the lower forearm and on the dorsum of the foot of four healthy volunteers. Three different provocations were conducted: local heating of the foot and systolic and venous occlusion of the upper forearm. Spectra were absolute calibrated using a microsphere solution linking measured spectra to Monte Carlo simulated spectra.

Correcting for inhomogeneously distributed blood significantly improved the data fitting in 13 out of 16 spectra and the estimated fraction of $\mathrm{Hb}$ increased $37 \%$ on average. Without compensation for inhomogeneously distributed blood, the model overestimated the influence of blood in the absorption coefficient and hence underestimated the fraction of blood. Including melanin significantly improved data fitting in 16 out of 16 spectra. The inclusion of melanin also affected the scattering parameters, $\alpha$ and $\beta$, and improved the ability for the model to mimic measured spectra. This improved ability was pronounced in the estimation of oxygen saturation during occlusion where the models including melanin estimated oxygen saturation to be close to zero, which is to be expected, and the model without melanin estimated the oxygen saturation to be $52 \%$ on average.

When using the single layer model including melanin and inhomogeneously distributed blood to fit spectra from the two source detector distances individually, the scattering parameter $\beta$ was consistently higher for the longer distance. In Figure 10.1, measured spectra together with the optimized model fit using one distance and evaluated at both distances is shown. The figure shows a 
good fit at the distance used in the model, while the model does not fit spectra at the other distance. The different $\beta$ for the two distances, was considered being reason that the model failed when fitting both source detector distances simultaneously, which in turn lead to erroneous results in the parameter estimates.
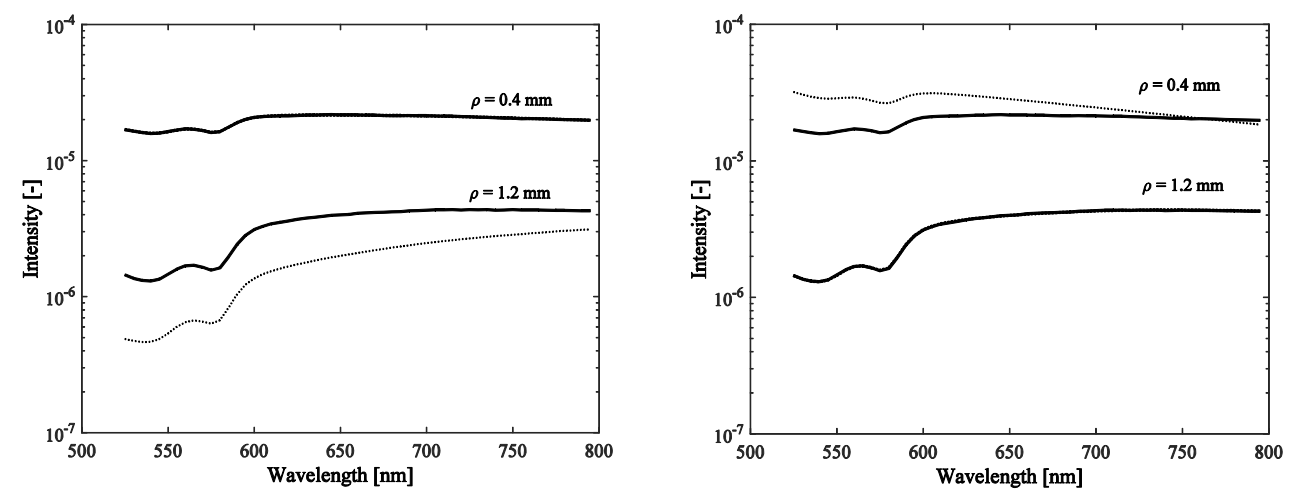

Figure 10.1. Absolute calibrated measured spectra (solid) and fitted (dotted) during heat provocation. Spectra are fitted to the shorter distance (left) and the longer (right) and evaluated at both distances.

Proposed ways to overcome this were a more advanced modeling of the skin including multiple layers with different optical properties, leading to a more realistic skin model (applied in paper III-V), or a more complex modeling of the scattering phase function (evaluated in paper II).

\subsection{PAPER II - INVERSE MONTE CARLO FOR ESTIMATION OF SCATTERING AND ABSORPTION IN LIQUID OPTICAL PHANTOMS}

In Paper II the effect of different scattering phase functions, the number of detecting fibers, the source detector distances in the range $0.2-1.2 \mathrm{~mm}$, and calibrations methods on the quantification of absorption and scattering in liquid optical phantoms were evaluated. Two scattering phase functions (Gegenbauer kernel (GK) and Henyey-Greenstein (HG)), three combinations of different number of detecting fibers (4, 2 or 1 fiber) and two intensity calibration methods were evaluated. Spectra were calibrated either by using a microsphere solution (absolute calibration) or a uniform illumination of the detecting fibers (relative calibration). Intralipid® and food dyes were mixed forming liquid optical phantoms with different scattering and absorption properties.

The results showed no significant improvement in the estimation of scattering and absorption when using the more complex GK phase function compared to the HG phase function. However, the rmserror was significant lower for the GK phase function and systematic residuals were not seen, in contrast to when the HG phase function was used. Even so, in more complex structures like human 
skin it may be impossible to estimate the wavelength dependent GK phase function. The simpler HG phase function with a constant anisotropy factor was, therefore, considered sufficient for accurate estimations of the absorption and scattering coefficients in the optical phantoms.

With an absolute calibration it was possible to accurately estimate both the absorption and the scattering using only one source detector distance. However this was only possible for the longer source detector distances $(\geq 0.46 \mathrm{~mm})$. For the shortest distance $(0.22 \mathrm{~mm})$ little information was provided in the SRDR spectra regarding chromophore content and hence the chromophore estimation was erroneous. The advantage with a relative calibration method over the absolute calibration is the simplicity, since the absolute calibration method involves a microsphere solution which is hard to characterize properly with the sufficient accuracy and consistency over time. Yet, a relative calibration method requires two source detector distances to estimate both absorption and scattering accurately.

Imperfection in the calculations of calibration factors and phantom mixing caused systematic deviations in rms-error (Figure 10.2) and inclusion of non-existing chromophores.
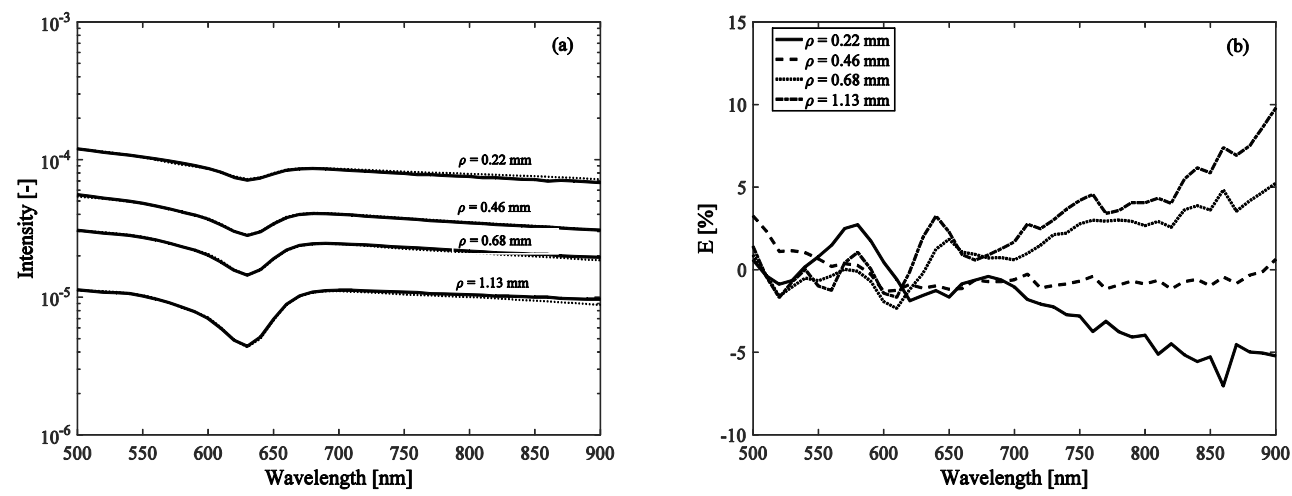

Figure 10.2. Fitted and measured spectra without intensity relaxation for a phantom including blue food dye and Intralipid (a). The systematic residual (b).

By allowing the intensity to vary in the optimization routine, the spectral fitting improved, systematic deviations were diminished and non-existing chromophores were not included, see Figure 10.3. 

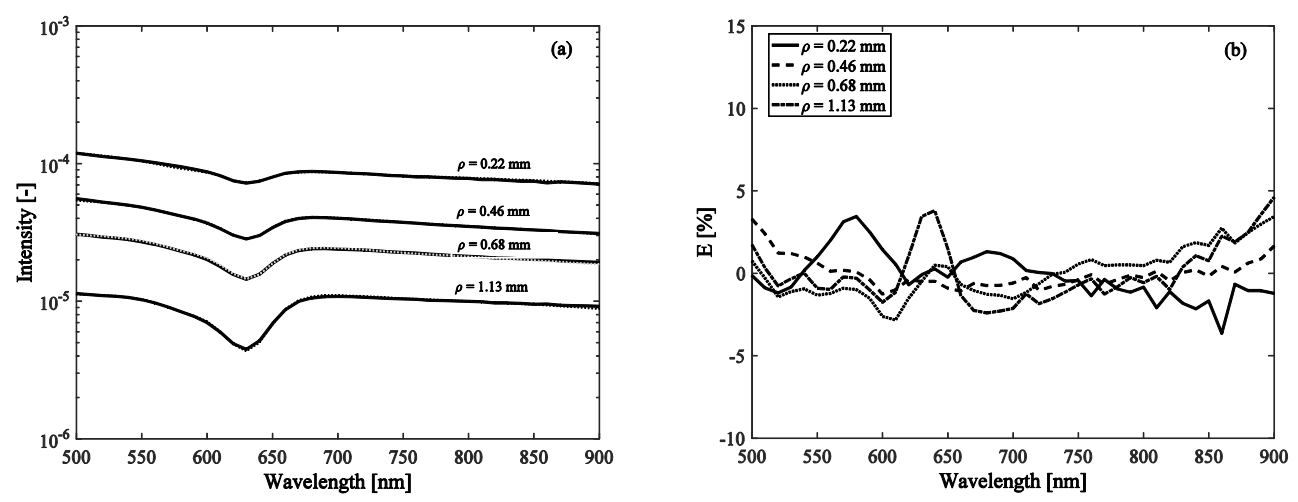

Figure 10.3. Fitted and measured spectra with intensity relaxation for a phantom including blue food dye and Intralipid (a). The residual (b).

This reduces the need for exact calibration factors and allows for small errors in the measurement setup, i.e. probe-tissue contact problems. However, the intensity relaxation factor may need to be controlled by boundaries or penalties in the data fitting, in order not to accept too large deviations between the intensities recorded at different fibers in relation to corresponding intensities in the modeled spectra. The intensity relaxation was implemented in paper III-V.

\subsection{PAPER III - MICROCIRCULATION ASSESSMENT USING AN INDIVIDUALIZED MODEL FOR DIFFUSE REFLECTANCE SPECTROSCOPY AND CONVENTIONAL LASER DOPPLER FLOWMETRY}

In paper III, DRS and LDF were integrated in a probe-based system and measurements were performed on the forearm of 33 healthy subjects during arterial occlusion. Based on paper II, a relative calibration procedure was used, a HG phase function described the scattering and two source detector distances were chosen. The one layer skin model used in paper I was extended to a three layer model describing DRS spectra. Three output parameters were given from the system: oxygen saturation, $\mathrm{RBC}$ tissue fraction and conventional perfusion. Oxygen saturation and RBC tissue fraction were expressed in absolute units [\%] by using an inverse Monte Carlo method for DRS, and tissue perfusion in perfusion units [PU] from analyzing LDF spectra in conventional way. The interrelationship between the output parameters was evaluated before, during and after the arterial occlusion.

Different sets with reduced number of model parameters in the spectral fitting, were statistically evaluated. The results showed that all model parameters significantly improved spectral fitting. The full model was hence further used to analyze measured spectra from the arterial occlusion. Time 
resolved perfusion, oxygen saturation and RBC tissue fraction from a typical occlusion are presented in Figure 10.4. Time points chosen for analysis are marked in the figure.
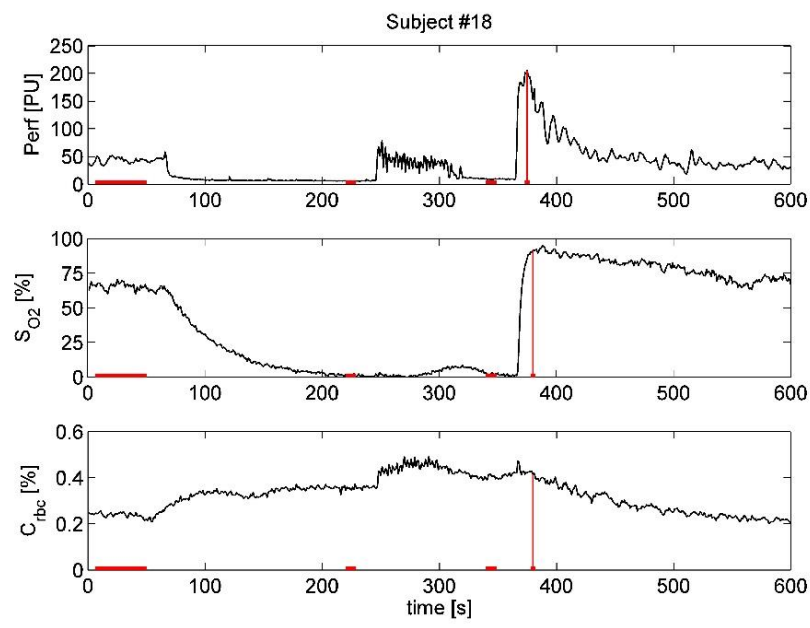

Figure 10.4. Time resolved perfusion, oxygen saturation SO2 and RBC tissue fraction $C_{r b c}$ for one of the subjects. Time points chosen for evaluation are marked.

The results showed a slow decrease in oxygen saturation after occlusion due to diffusion of oxygen to the tissue compared to the immediate decrease in perfusion. The finding of a larger increase in perfusion compared to RBC tissue fraction at release indicates an increased average RBC speed, which can be assumed to arise in larger vessels with a higher average RBC speed. By combining DRS and LDF and assess oxygen saturation, perfusion and RBC tissue fraction simultaneously and in essentially the same vascular bed gives new possibilities to interpret physiological reactions. The next step to explore the parameters from an integrated model for both DRS and LDF was presented in paper IV.

\subsection{PAPER IV - OXYGEN SATURATION, RED BLOOD CELL TISSUE FRACTION AND SPEED RESOLVED PERFUSION - A NEW OPTICAL METHOD FOR MICROCIRCULATORY ASSESSMENT}

In paper IV, DRS and LDF were fully integrated in an individually adaptive skin model and applied in vivo. Monte Carlo simulated spectra were compared to measured spectra in an optimization routine and the output parameters oxygen saturation, speed resolved perfusion and RBC tissue fraction were given in absolute units. The output parameters were estimated in 23 healthy subjects during local heating of the foot and during venous and arterial occlusion of the arm. The output parameters were assessed simultaneously and in the same skin site. The aim was to explore the output parameters in 
baseline, in different skin sites and during different provocations that are often used in skin physiology studies.

In some of the measurements, the probe was placed on a region with a small amount of blood. In these cases the spectral signature of hemoglobin was very weak and estimated oxygen saturation became uncertain. By introducing an exclusion criteria on the size of the hemoglobin spectral area in the 506-614 wavelength region related to the total area, these spectra with poor signature were excluded.

The estimated parameters during the provocation were comparable to other studies where DRS and LDF were used separately. By dividing the perfusion in different speed regions it was possible to follow the response for the different regions during the provocations, and in combination with the oxygen saturation gain new insight into the microcirculatory system. During local heating there was a larger increase in the perfusion for higher speeds compared to the lowest speed region (Figure 10.5). In combination with the strong correlation between change in perfusion for higher speeds and change in oxygen saturation after heating these finding can be related to a non-metabolic flow after a thermal provocation. Time to peak after release of arterial occlusion differed between the different perfusion speed regions. Also the oxygen saturation had a slower time to peak than total perfusion and the return to baseline values was slower than the RBC tissue fraction.
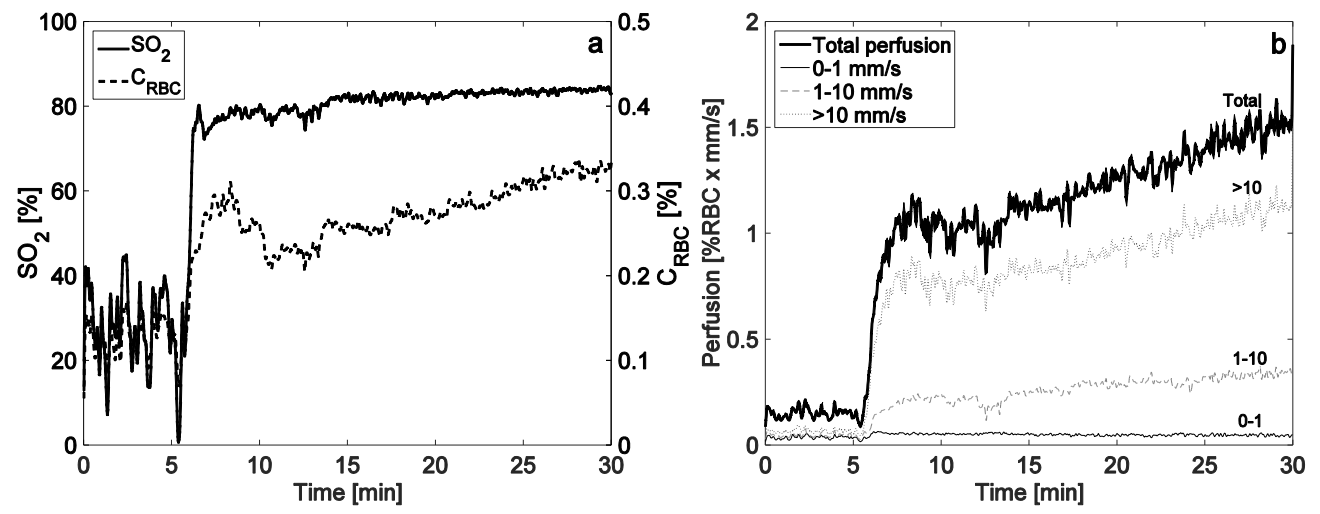

Figure 10.5. Oxygen saturation and RBC tissue fraction (a) and speed resolved perfusion (b) during local heating. 


\subsection{PAPER V - TYPE 2 DIABETES IS ASSOCIATED WITH IMPAIRED MICROVASCULAR FUNCTION IN THE SKIN INDEPENDENTLY OF MICROALBUMINURIA}

The integrated method applied on healthy subjects in paper IV, was used to evaluate patients with diabetes and controls in paper V. Since the albumin/creatinine ratio (ACR) is a marker of kidney disease and a sign for microvascular dysfunction in diabetes mellitus, the patients with diabetes were divided into two groups; without an elevated ACR (no microalbuminuria) and with an elevated ACR (microalbuminuria). In total, the study included 120 subjects; 41 controls, 38 patients with diabetes but without microalbuminuria and 41 patients with diabetes and microalbuminuria. Oxygen saturation, speed resolved perfusion and RBC tissue fraction during local heating of the foot together with common clinical data were evaluated.

The microvascular response to heating was assessed at baseline, during the first peak upon heating (associated with sensory nerves) and at the plateau phase 25 minutes after heating onset (associated with nitric oxide (NO)). In baseline, the perfusion for speeds $0-1 \mathrm{~mm} / \mathrm{s}$ and the RBC tissue fraction were reduced in patients with type 2 diabetes, both with microalbuminuria and without. Also, first peak in total perfusion was reduced in type 2 diabetes. RBC tissue fraction correlated to HbA1c both in baseline and at plateau, and total perfusion at first peak correlated to $\mathrm{HbAlc}$.
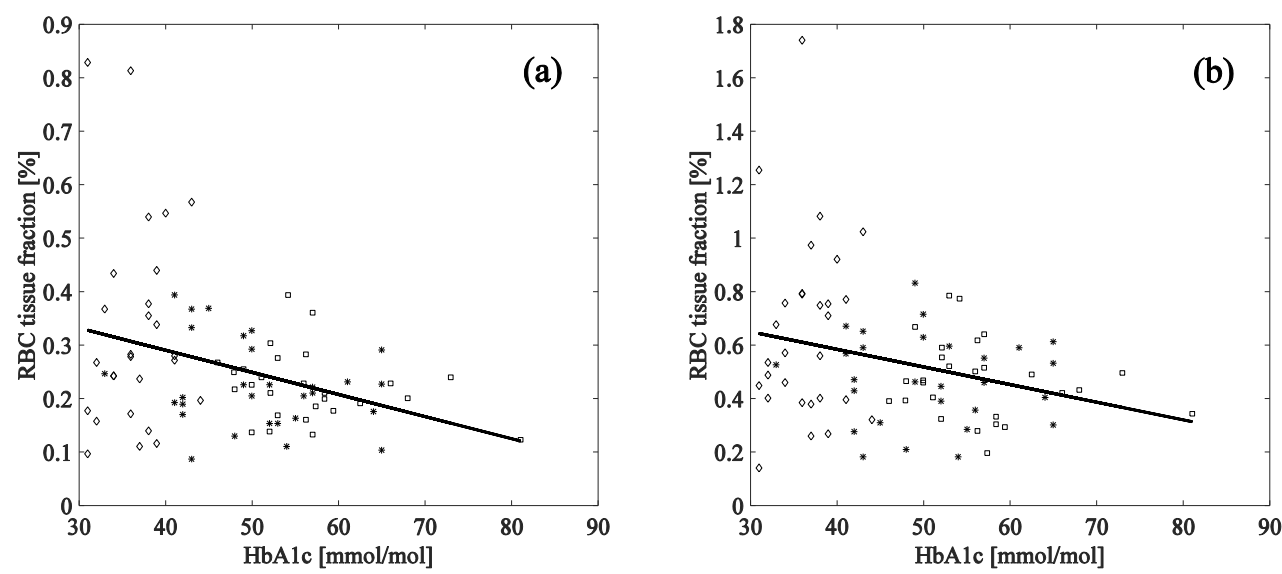

Figure 10.6. Correlation between RBC tissue fraction and HbAlc during baseline (a) and plateau (b). Controls (diamonds), patients with diabetes type 2 without microalbuminuria (star) and patients with diabetes type 2 with microalbuminuria (square).

During baseline, subjects with diabetes had a smaller proportion of the total flow distributed in the lowest speed region compared to controls, and hence the average speed for patients with diabetes, was higher. The microvascular reactivity correlated to HbA1c in several parameters, showing the relationship between hyperglycemia and endothelial function. These findings show the usefulness of 
Chapter 10 - Review of the papers

differentiating between different speed regions, to address the smallest vessel structure of relevance to diabetic pathogenesis. The conclusion of the study was that cutaneous microcirculation is impaired in the foot in type 2 diabetes, independently of microalbuminuria. 


\section{DisCUSSION}

This thesis describes the development and evaluation of an integrated DRS and LDF probe system for quantifying skin microcirculatory blood flow and oxygen saturation. In the following subsections, tissue model considerations, spectral fitting and preprocessing, the given output parameters from the model and their clinical applications are discussed.

\subsection{THE TISSUE MODEL}

The skin is a heterogeneous structure. It includes many layers where each layer has different optical properties. Two strong absorbers in the visible range are melanin and hemoglobin, and in the skin they reside in different layers. However, several skin models for DRS are based on single-layer models where the chromophores are homogenously distributed [13, 72, 107]. Hennessy et al [108] evaluated a single-layer skin model on two-layer model data for DRS and showed that a single-layer model lead to erroneous parameter estimations regarding fraction of melanin, fraction of hemoglobin and oxygen saturation, even if correcting for inhomogeneously distributed blood. Their single-layer model underestimated melanin and hemoglobin concentration, and the magnitude of the underestimation was found to be a function of the epidermal thickness of the two-layered data. Our results show that a single-layer model for skin with homogenously distributed blood estimated especially the oxygen saturation incorrectly. Fredriksson et al [104] showed similar results, where a homogenous single-layer model gave poor spectral fit and incorrect oxygen saturation estimations. Furthermore, the single-layer model failed to describe spectra from multiple source-detector distances, as presented in paper I.

The extension of a single-layer model to a two-layered model for DRS has been used by several authors [109-111]. By having blood and melanin separated in two layers, the model is able to describe skin more accurately than a single-layer model. The skin model presented in this thesis is an extension to a three-layer model, with the ability to more realistically mimic the blood distribution in skin. With a three-layer model i.e. two layers including blood, the oxygen saturation and the fraction of blood can be allowed to differ between the two layers. Since the size of the vessels can be assumed to increase with depth and hence also the oxygen saturation and RBC tissue fraction, a three-layer model can reflect contribution from different vessel types in the parameters. This is not possible with a twolayer model i.e. only one layer including blood. 
In the presented three-layer model, the scattering is equal in all layers. Other groups have used a twolayered model with different absorption and reduced scattering coefficients in the two layers [109, $112,113]$, but they all had difficulties to estimate the optical properties of the top layer accurately. Wang et al. [112] showed large deviations between the true and estimated absorption and reduced scattering coefficient in the top layer of a two layered phantom, when the top layer thickness was below $400 \mu \mathrm{m}$, especially in the reduced scattering coefficient. Also Liu and Ramanujam [109] had difficulties estimating the optical properties of the top layer when the thickness was below $200 \mu \mathrm{m}$. A thin top layer will have a shorter photon path length traveled within the layer and hence result in less information about the optical properties.

A model for the reduced scattering coefficient with separate Mie and Rayleigh scattering parts was tested in paper III, and was shown to be significantly better than the single-component scattering model $\mu_{s}^{\prime}=\alpha\left(\lambda / \lambda_{0}\right)^{-\beta}$. Tseng et al. [114] found that a single-component scattering expression did not fit spectra well in the 500 to $1000 \mathrm{~nm}$ wavelength range and a two-component scattering expression was better. The Mie scattering component reflects the scattering from collagen fibers while the Rayleigh scattering component reflects the contribution to the scattering by smaller structures in the skin like collagen fibrils [58]. Bashkatov et al. [115] showed that in the wavelength range of 400 to $600 \mathrm{~nm}$ the Rayleigh scattering contribution to the reduced scattering coefficient is large, while for longer wavelengths becomes negligible, and hence including the Rayleigh scattering component is necessary in the visible wavelength range. The combined Mie and Rayleigh scattering expression for the reduce scattering coefficient was considered to be the best descriptor in the wavelength range of 400 to $2000 \mathrm{~nm}$, [115].

There are different ways to model the absorption of tissue. In this thesis, the absorption coefficient was modeled as a linear combination of chromophores and the fraction of each chromophore was estimated in the fitting of spectra. Therefore, when fitting our model to measured spectra, wavelength constraints are present for the absorption coefficient (given by the chromophore absorption spectra). A principally different method is that using spatially modulated light, where the absorption coefficient is estimated for each wavelength independently. Hence, there is no assumption of the spectral shape of the absorption coefficient [93]. The chromophore content is then fitted in the post processing to the estimated absorption at each wavelength. This approach demands calibration against a reference phantom with known optical properties and no intensity relaxation can be applied in the inverse fitting.

Other absorbers in skin which can be included into the model are for example water, fat, bilirubin and beta-carotene. Water is a common chromophore to include [41, 42, 114] and was included as a chromophore in paper I. However, water has small influence below $750 \mathrm{~nm}$ and hence the impact on the detected intensity in our wavelength range $(450-850 \mathrm{~nm})$ is limited. To be able to estimate the fraction of water, wavelengths above $900 \mathrm{~nm}$ are used where water has more dominant absorption [114]. In paper II, the absorption coefficient for water was added when using SCT for determining the absorption coefficient for the dyes, but there was no difference in the estimation of the absorption 
coefficient with or without water absorption. Bilirubin and beta-carotene absorb mostly below 500 $\mathrm{nm}$ where few wavelengths are used in our system. Misfits between measured and simulated spectra can originate from other chromophores not included into the skin model. By analyzing the error from the spectral fitting it is possible to get indications of missing chromophores.

The importance of an inhomogeneous modeling of blood vessels for DRS was shown in paper I and III, and has also been shown in several other studies [97, 101, 103], where a homogenous model overestimated the influence of blood in the absorption coefficient and hence underestimated the fraction of blood. The vessel packaging effect in LDF has not been investigated as much as for DRS. When a photon is Doppler shifted, inhomogeneous modeling of blood vessels will cause consecutive shifts to be caused by RBCs with similar speed and this will alter the Doppler shift distribution [99]. The vessel packaging effect for LDF will also cause less light to be Doppler shifted compared to an equal RBC tissue fraction homogenously distributed, and the light that is Doppler shifted will be multiple shifted to a greater extent [99].

\subsection{SPECTRAL PREPROCESSING AND FITTING}

The ability of the model to mimic measured spectra well is essential for reliable output parameters. However, if there is a lack of quality in measured spectra, the model cannot produce similar spectra. Noise might be added to measured spectra, for example movements and probe-tissue contact problems. There can also be errors introduced in the calibration procedure of measured spectra. If there is no hemoglobin signature in DRS spectra, the model cannot estimate the oxygen saturation accurately. All these factors are preferably found with exclusion criteria before the fitting, otherwise fitting parameters could try to compensate for the mismatch and herby produce erroneous estimates. A straightforward exclusion criterion is to evaluate the residual from the fitting where spectra with too large fitting error are discarded. With this type of exclusion criterion the origin of the misfit is not investigated, but it may catch the fitting errors due to probe-tissue contact issues. Häggblad et al. [116] used an exclusion criterion for poor probe-tissue contact by discarding DRS spectra where the intensity at $640 \mathrm{~nm}$ varied more than $30 \%$ from the median intensity. This type of exclusion criteria could be used in baseline measurements where intensity variations could be assumed to be small. During an occlusion provocation or a local heating, this exclusion criteria can be harder to apply, since it is possible that the intensity will change due to the provocation. Movement artifacts are easily detected in measured Doppler power spectra since they cause peaks in the normally monotonically decreasing spectra.

When DRS spectra had barely no hemoglobin signature, the method estimated the oxygen saturation parameter to be close to and below zero. During baseline, these estimates were clearly not accurate and associated with a low RBC tissue fraction. A way to distinguish spectra with low fraction of hemoglobin before solving the inverse problem was therefore presented in paper IV. The hem- 
exclusion criteria discarded spectra where the hemoglobin area was too small in relation to the total spectral area. In this way it was possible to exclude spectra based on other premises than the output parameters (low RBC tissue fraction or oxygen saturation).

To perform an absolute intensity calibration of spectra, a stable and accurately characterized calibration standard is necessary. Common errors and imperfections in the measurement setup for all detector distances such as intensity variations in the lamp and poor probe-tissue contact will introduce errors in calibrated spectra, and the fitting of absolute calibrated spectra and the estimated parameters will be affected. Aggregation of microspheres will also affect the scattering properties of the microsphere solution and also the absolute calibration factors. In a relative calibration procedure, such common errors are eliminated.

The benefit with absolute calibration of spectra is the possibility to estimate the absorption and the scattering coefficient using only one source-detector distance. In paper II, the rms-error of the estimated scattering coefficient, compared to the true, was lower for the absolute calibrating method compared to the relative calibration method for all numbers of included detector distances and phase functions. However the improvement in the estimation of the absorption coefficient was minimal. It was also shown in paper I that a simple homogenous tissue model was sufficient for modeling DRS spectra at a single-distance. With multi-distance measurements, which are needed for a relative calibration of spectra, a more realistic tissue model is necessary.

Simulated spectra are derived based on assumptions on geometry and optical properties of tissue. With small source detector distances, small errors in the geometry of the probe or other geometries in the simulation will have an effect on simulated spectra and hence also the optimization process. By measuring the geometries as correct as possible, these errors can be minimized. In addition, the intensity relaxation factor into the spectral fitting allows for minor deviations between simulated and true geometry. The inclusion of the intensity relaxation factor $q$ in paper II significantly improved the ability of relative and absolute calibrated models in paper II to accurate estimate especially the scattering. The fitting also generated a lower residual without systematic deviations for both calibration methods. Fredriksson et al [99] also found that the inclusion of $q$ increased the accuracy of the estimation of oxygen saturation, the RBC tissue fraction and the total perfusion in simulated models. In the ideal case, $q$ is unity. Too large deviations from unity need to be handled. If $q$ is allowed to take any value, multiple solutions is a possible outcome and convergence of the method cannot be assured. In paper II and III, deviations was handled with upper and lower limits of $q$. In paper IV and $\mathrm{V}$, deviations from unity in $q$ were instead penalized in the optimization routine. A large deviation from unity of $q$ indicates that, in some way, the model cannot fully explain measured spectra. Fredriksson et al. [99] found the largest deviation of $q$ in simulations with a high concentration of blood vessels located right below the bloodless layer. In these simulations the 
accumulation of blood vessels was found in a layer much thinner than the $0.5 \mathrm{~mm}$ layer, and an adaptive layer thickness was suggested as solution.

\subsection{THE OUTPUT PARAMETERS}

The estimated output parameters are based on a skin model, simulations and a non-linear optimization algorithm. Oxygen saturation values from the presented method were comparable to values obtained from other studies during baseline, at peak after arterial occlusion and after heating [14, 117, 118]. The perfusion values were more difficult to compare to other studies since the source-detector distance used for LDF in the method differs from the standard probe distance. With a different sourcedetector distance, the sampling depth differ and hence change the distribution of sampled blood vessels. The time to peak after arterial occlusion for the total perfusion was similar to findings by Yvonne-Tee et al [119].

In paper II, two source detector distances and a relative calibrated method for DRS showed an estimation of chromophore content and scattering coefficient deviating within on average $10 \%$ from the true value. The highest error $(18.5 \%)$ in the chromophore estimation was for the phantom with the lowest absorption coefficient, $\mu_{a}=0.055 \mathrm{~mm}^{-1}$ at $520 \mathrm{~nm}$, which is to be expected since a weak absorption signature makes it harder for the model to estimate the absorption. If the criterion on the hemoglobin spectral area, applied in paper IV and V, would have been implemented in paper II, the spectrum from this phantom would have been considered to have a too weak blood signature and had been rejected.

The oxygen saturation parameter is calculated from oxygenized and deoxygenized hemoglobin spectra, and it will therefore only reflect the amount of oxygen bound to hemoglobin. There is a distinction from the tcpO2 value which measure the amount of oxygen that has diffused from the capillaries through the epidermis to the electrode attached on the skin surface. Oxygen in blood can be dissolved in plasma and bound to hemoglobin, where hemoglobin has a maximum capacity of carrying 4 oxygen molecules. This difference will be pronounced during for example a breathing provocation with oxygen. While breathing $100 \%$ oxygen, the oxygen saturation parameter will rapidly reach a plateau where all possible oxygen is bound to hemoglobin, while the tcpO2 value will continue to increase [120]. Also it should be noted that the tcpO2 electrode heats the skin, causing a local hyperemia with an increased blood flow and oxygen pressure as a result. Thus, the tcpO2 value is not representative as a baseline value of tissue oxygenation.

When modeling skin, there is a fine balance between having enough parameters to accurately describe measured spectra and a having a minimal model with as few parameters as possible. The importance of the model parameters was evaluated in paper I and III, and has also been evaluated by Fredriksson et al. [104]. All parameters were shown to significantly improve model fitting. Several of the 
parameters in the model are not presented as output parameters. Parameters used in the skin model like the epidermal thickness and vessel diameter would be interesting to study and get as output parameters from the method. However, the main purpose of the method was not to estimate these parameters and their accuracy has not been evaluated. Also, the objective function in the optimization algorithm was designed to estimate the RBC tissue fraction and the oxygen saturation as accurate as possible. If other parameters are interesting to get as output parameters, the objective function needs to be designed based on other premises. Even so, the other model parameters can indicate how well the model can describe real tissue. In paper I, the higher $\beta$ in the scattering parameters for the longer source detector distances was found as one reason why a one-layer model could not be used to fit spectra from two source-detector distances. Also in paper I, the average vessel radius from the shorter source-detector distance was smaller than the longer distance for all provocation, which would be expected with an accurate model since the sampling volume is smaller.

Due to the wavelength dependent absorption and scattering in tissue, different wavelengths penetrate tissue with different depths. Shorter wavelengths will generally have a shallower penetration depth than longer wavelengths [45]. Tseng et al. [114] separated the DRS fitting into two wavelength regions (500-600 $\mathrm{nm}$ and 600-1000 $\mathrm{nm}$ ) to see depth differences in oxygen saturation. Saager et al. [121] estimated the epidermal thickness and melanin concentration by estimating the penetration depth for different wavelengths and separating the wavelengths into two regions (450-605 nm and 784-1000 nm). To extend the wavelength range up to $1000 \mathrm{~nm}$ in our system would be interesting to be able to distinctly separate the epidermal layer from the dermis layers and maybe get a more accurate estimation of the epidermal thickness. To expand the wavelength range for our system, the sensitivity of the spectrometers need to be higher. Also, longer wavelengths can reach larger vessels since the sampling depth is larger, and this could be an advantage or a disadvantage.

The estimated sampling volume will have an effect on the estimated RBC tissue fraction and speed resolved perfusion, since these parameters are heterogeneously distributed in the tissue model and average values over the sampling volume are presented. Also, the oxygen saturation in paper $\mathrm{V}$ is calculated from the sampling volume. The sampling volume includes a mixture of capillaries, venules and arterioles, all contributing to the estimated parameters. In paper III-V, the sampling volume used to calculate the average RBC tissue fraction and speed resolved perfusion was based on the best fit model after the spectral fitting, reflecting the actual volume the photons have propagated in. An individual average sampling volume can be obtained from each measurement and the output parameters are calculated from the actual sampling volume. Fredriksson et al. [99] used a fixed 3 $\mathrm{mm}^{3}$ half sphere sampling volume, with a radius of 1.13 and centered at the white light emitting fiber based on simulations and expected measurement volumes for the source-detector distances. With a fix sampling volume, it could be easier to compare different measurements, but large blood vessels located outside the half sphere can influence spectra and leading to an overestimation of the parameters. 
A point measurement cannot be assumed to have the same blood flow and oxygen saturation as the tissue on average. Conventional LDF has therefore been considered a technique with poor spatial reproducibility to assess microvascular perfusion [122]. The spatiotemporal variability in the parameters was assessed in paper IV and also from additional data obtained in the study presented in paper $\mathrm{V}$, see section 9.5.2. The oxygen saturation and RBC tissue fraction was the parameters with the lowest variability, below $30 \%$ at both arm and foot. However, these reproducibly measurements reflects both spatial variability and temporal variability and the number of measurement sites is limited. To separate spatial and temporal variability with multiple probes, repeated measurements on the same skin site or an increased number of measurement sites would give a more detailed description of the reproducibility of the method. Further investigations regarding reproducibility of the method are planned in future studies.

Combining information about blood flow and blood oxygen saturation is a promising tool. Sunar et al. [123] combined diffuse correlation spectroscopy (DCS) with DRS to obtain blood flow and oxygen saturation in tumors to quantify the response to therapy. However, the source detector distance of 3 $\mathrm{cm}$ needed to assess blood flow with DCS yield a sampling depth of approximately $1 \mathrm{~cm} \mathrm{[123].} \mathrm{At}$ this depth, larger vessels will contribute to the signal in a larger extent than the developed system in this thesis. In cerebral studies, a measure on the cerebral metabolic rate of oxygen $\left(\mathrm{CMRO}_{2}\right)$ is often calculated by measuring both blood flow and oxygen saturation. Verdecchia et al. [124] combined DCS with NIRS to quantify $\mathrm{CMRO}_{2}$ in pigs, but to extend the technique to patients a multilayered tissue model was considered necessary to obtain depth resolved blood flow and oxygen saturation. Zhou et al. [125] combined diffuse optical spectroscopy (DOS) with DCS and calculated a mammary metabolic rate of oxygen $\left(\mathrm{MMRO}_{2}\right)$ for estimation of the ratio tumor/normal tissue by using the model for $\mathrm{CMRO}_{2}$. The source detector distance in this study was also $\sim 3 \mathrm{~cm}$ inducing a larger sampling volume compared to the system in this thesis.

\subsection{CLINICAL APPLICATIONS AND FUTURE RESEARCH}

Simultaneous measurements of oxygen saturation, RBC tissue fraction and speed resolved perfusion enable new possibilities to study the interrelationship between the parameters. That enables deeper insight in the function of the microcirculation both during normal and pathological conditions. In paper III, the oxygen saturation decreased slowly upon occlusion of the arm by diffusion of oxygen to the tissue, while the blood flow assessed by conventional perfusion decreased fast. At hyperemia, both tissue fraction of RBC and oxygen saturation increased due to vasodilation. By analyzing the conventional perfusion, the increase in perfusion was higher than the increase in RBC tissue fraction indication a higher average speed of the RBCs. Vasodilation could therefore be assumed to be predominant in larger vessels with a higher average speed. Adingupu et al. [27] reported an abnormal reperfusion pattern after ischemia that resulted in a deranged oxygen saturation pattern. By measuring 
both oxygen saturation and conventional perfusion, the microvascular dysfunction in perfusion and the consequence on oxygen saturation could be directly visualized.

The separation of perfusion into speed regions allows for a more detailed description of blood flow. In paper IV, the perfusion increase from baseline at thermal hyperemia was different for the three speed regions, where the highest increase was found in the highest speed region. The difference was assumed to be related to the different vessels involved in the different speed regions. The lowest speed region consists primarily of capillaries with a smaller vasodilating ability compared to larger vessels (associated with the highest speed region). Fredriksson et al. [126] associated the increase in the highest speed region at thermal hyperemia to a shunt flow, where the blood was shunted from the arterial to the venous side. In paper IV, the increase in oxygen saturation and speed resolved perfusion measured simultaneously showed a correlation between oxygen saturation increase and changes in perfusion for higher speeds. This support the assumption of an increased non-metabolic flow in the larger vessels at thermal provocation.

Maximal vasodilation is obtained when heating the skin to $42-44^{\circ} \mathrm{C}$. The first peak in perfusion at thermal hyperemia has been associated with sensory nerves and the plateau mostly with NO ( 60\%) [32]. However, other factors like endothelium-derived hyperpolarization factors (EDHF) contributing to the thermal response when heating to this temperature [127]. At a temperature of $44^{\circ} \mathrm{C}$ it is hard to separate the NO- and the EDHF-dependency without for example iontophoresis [128]. A reduced temperature of $39^{\circ} \mathrm{C}$ has therefore been proposed by Choi et al. [128], where the plateau will be dominated $(>80 \%)$ by NO. Also, heating at a temperature of $>42^{\circ} \mathrm{C}$ can cause a sense of pain, leading to activation of other mechanisms inducing vasodilation. Kellogg et al. [129] showed that even a small sense of pain caused the vasodilation to remain even in the presence of nitric oxide synthase inhibitors, indicating other involving mechanisms. Carter and Hodges [130] reported an absence of initial peak and nadir when heating the skin rapidly to $44^{\circ} \mathrm{C}$, where all subjects reported a short period of mild pain. Even if none of the participants in the studies in paper IV and V reported a sense of pain, a first peak and nadir were absent in several subjects in paper $\mathrm{V}$. This indicates that the chosen temperature of $44^{\circ} \mathrm{C}$ in the study had probably caused minor pain and a temperature of $\left\langle 41^{\circ} \mathrm{C}\right.$ is probably a better choice in future studies.

Diabetes mellitus type 2 is associated with microvascular changes and endothelial dysfunction. By presenting parameters in absolute units it is possible to assess the microvasculature without performing provocations of the tissue. Wojtkiewicz et al. [131] presented perfusion values in absolute units by decomposition of laser Doppler spectrum and showed significant higher mean RBC speed in baseline in diabetes type 1 compared to controls. It is in line with the findings in paper $\mathrm{V}$, where the population with diabetes had less of the total perfusion distributed in the lowest speed region and hence the average speed was higher than controls. Also, the baseline flow in the lowest speed region and the RBC tissue fraction were significantly lower for diabetes type 2 compared to controls. It 
shows the usefulness of separating the perfusion into different speed regions and to combine LDF with DRS to obtain information about the nutritive blood flow.

Today, skin microcirculatory function is not used as a diagnostic marker or a predictor of diabetes or cardiovascular diseases. To discover the potential of skin microcirculation, the microcirculatory function and its correlation to other vascular measurements needs to be investigated in larger, standardized studies [7]. This integrated system is a new tool for assessing microvascular dysfunction and is recently commercial available for research by the Periflux 6000 EPOS (enhanced perfusion and oxygen saturation) system. The system is currently in use in the Swedish CardioPulmonary BioImage Study (SCAPIS) with the overall goal to find risk factors for cardiovascular diseases. By combining measurements on heart, lungs and blood vessels with measurements of the microcirculation, it is possible to relate skin microvascular function to heart and large vessel function. This non-invasive, real-time system could then potentially replace high cost measurements such as magnetic resonance imaging (MRI) and computed tomography (CT) with less expensive and easier measurement. 



\section{Conclusions}

A fiber optic system, integrating diffuse reflectance spectroscopy and laser Doppler flowmetry is proposed and evaluated.

The three-layer model for DRS and LDF with compensation for inhomogeneously distributed blood was evaluated where all reported parameters in the model were found necessary to describe measured spectra. A relative calibration procedure with two source-detector distances for DRS was found adequate to able to estimate the absorption and scattering properties of optical phantoms within $10 \%$. By introducing an intensity relaxation in the fitting of simulated spectra to measured spectra, allowing for measurement imperfections, spectral fitting and parameter estimation were improved.

Application of the system on healthy subject and in patients with diabetes type 2 revealed new possibilities to gain extended knowledge of the microcirculation. In diabetes type 2, the RBC tissue fraction and nutritive flow are reduced independently of other signs of vascular disease. With quantitative assessment of oxygen saturation, RBC tissue fraction and speed resolved perfusion, this system will aid in assessment of the vital microcirculatory function. 



\section{ACKNOWLEDGEMENTS}

The studies in this thesis were financed by VINNOVA and Perimed AB through the SamBIO research collaboration program between companies and academia within bioscience, and through the Research and grow programme, by NovaMedtech, supported by the European Union Regional Development Fund, and by Perimed AB and Linköping University through the Center for Excellence NIMEDCBDP (Center for Biomedical Data Processing). I have also been supported by AgoraLink.

Thanks to all participants in my studies, without your help this wouldn't been possible.

To Tomas Strömberg, my main supervisor and friend. Thanks for your never ending support and positive spirit. I have received the best guidance!

Thanks to Marcus Larsson and Ingemar Fredriksson, my supervisors and highly appreciated mentors who always found the time to help me or just let me talk for a while.

Göran Salerud, thank you for believing in me and helping me start my journey. And also for being you.

To Sara Bergstrand, for valuable discussions and support. I have always left our meetings with new positive energy!

Thanks to Carl Johan Östgren, for great assistance and making me feel welcome in the CARDIPP community.

Thanks to Perimed AB for the cooperation and engagement. Especially to Anders Pettersson for your help and guidance.

All colleagues at the Department of Biomedical Engineering, for making every workday a good day.

To my former colleagues Martin, Joakim, Marie and Elin, you are missed.

Thanks to Maria, Johannes, Peter, Linda, Fabiola and Neda, for giving me valuable input on this thesis.

To my friends and family. Mamma och pappa, tack för att ni alltid har uppmuntrat mig och låtit mig göra mina egna val. Ni är fantastiska! Till mina systrar Helena och Susanna, ni finns alltid där för mig!

Till Jimmy, Matilda och Elicia. Jag älskar er! 



\section{REFERENCES}

1. Davis, M.J., M.A. Hill, and L. Kuo, Chapter 6 - Local Regulation of Microvascular Perfusion, in Microcirculation (Second Edition), R.F.T.N.D. Ley, Editor. 2008, Academic Press: San Diego. p. 161284.

2. Abularrage, C.J., et al., Evaluation of the microcirculation in vascular disease. Journal of Vascular Surgery, 2005. 42(3): p. 574-581.

3. Brunner, H., et al., Endothelial function and dysfunction. Part II: Association with cardiovascular risk factors and diseases. A statement by the Working Group on Endothelins and Endothelial Factors of the European Society of Hypertension. Journal of Hypertension, 2005. 23(2): p. 233-246.

4. Levy, B.I., et al., Impaired tissue perfusion a pathology common to hypertension, obesity, and diabetes mellitus. Circulation, 2008. 118(9): p. 968-976.

5. Rossi, M. and A. Carpi, Skin microcirculation in peripheral arterial obliterative disease. Biomedicine \& Pharmacotherapy, 2004. 58(8): p. 427-431.

6. Holowatz, L.A., C.S. Thompson-Torgerson, and W.L. Kenney, The human cutaneous circulation as a model of generalized microvascular function. Journal of Applied Physiology, 2008. 105(1): p. 370372.

7. Hellmann, M., M. Roustit, and J.-L. Cracowski, Skin microvascular endothelial function as a biomarker in cardiovascular diseases? Pharmacological Reports, 2015. 67(4): p. 803-810.

8. Muris, D.M., et al., Microvascular Dysfunction Is Associated With a Higher Incidence of Type 2 Diabetes Mellitus A Systematic Review and Meta-Analysis. Arteriosclerosis, thrombosis, and vascular biology, 2012. 32(12): p. 3082-3094.

9. Roustit, M. and J.L. Cracowski, Non-invasive Assessment of Skin Microvascular Function in Humans: An Insight Into Methods. Microcirculation, 2012. 19(1): p. 47-64.

10. Milstein, D.M.J., R. Bezemer, and C. Ince, Sidestream Dark-Field (SDF) Video Microscopy for Clinical Imaging of the Microcirculation, in Microcirculation Imaging. 2012. p. 37-52.

11. De Backer, D., et al., Monitoring the microcirculation in the critically ill patient: Current methods and future approaches. Intensive Care Medicine, 2010. 36(11): p. 1813-1825.

12. Durduran, T., et al., Diffuse Optics for Tissue Monitoring and Tomography. Rep Prog Phys, 2010. 73(7).

13. Palmer, G.M., et al., Monte Carlo-based inverse model for calculating tissue optical properties. Part II: Application to breast cancer diagnosis. Applied optics, 2006. 45(5): p. 1072-8.

14. Kuliga, K.Z., et al., Dynamics of Microvascular Blood Flow and Oxygenation Measured Simultaneously in Human Skin. Microcirculation, 2014. 21(6): p. 562-573.

15. Forst, T., et al., Reliability of lightguide spectrophotometry $\left(\mathrm{O}^{2} \mathrm{C}^{\circledR}\right)$ for the investigation of Skin tissue microvascular blood flow and tissue oxygen supply in diabetic and nondiabetic subjects. Journal of Diabetes Science and Technology, 2008. 2(6): p. 1151-1156.

16. Fredriksson, I., M. Larsson, and T. Strömberg, Model-based quantitative laser Doppler flowmetry in skin. Journal of biomedical optics, 2010. 15(5): p. 057002.

17. Lindbergh, T., Quantitative diffuse reflectance spectroscopy: myocardial oxygen transport from vessel to mitochondria. 2009.

18. Liebert, A., N. Zołek, and R. Maniewski, Decomposition of a laser-Doppler spectrum for estimation of speed distribution of particles moving in an optically turbid medium: Monte Carlo validation study. Physics in Medicine and Biology, 2006. 51(22): p. 5737-5751.

19. Tortora, G.J. and B.H. Derrickson, Principles of Anatomy and Physiology. 2006: Wiley.

20. Whitton, J.T. and J.D. Everall, The thickness of the epidermis. British Journal of Dermatology, 1973. 89(5): p. 467-476. 
21. Gambichler, T., et al., In vivo data of epidermal thickness evaluated by optical coherence tomography: Effects of age, gender, skin type, and anatomic site. Journal of Dermatological Science, 2006. 44(3): p. 145-152.

22. Braverman, I.M., The cutaneous microcirculation. Journal of Investigative Dermatology Symposium Proceedings, 2000. 5(1): p. 3-9.

23. Guyton, A.C., Textbook of medical physiology. 8th ed. 1991, Philadelphia: Saunders.

24. Minson, C.T., Thermal provocation to evaluate microvascular reactivity in human skin. Journal of Applied Physiology, 2010. 109(4): p. 1239-1246.

25. Johnstone, M.T., et al., Impaired endotheliuni-dependent vasodilation in patients with insulindependent diabetes mellitus. Circulation, 1993. 88(6): p. 2510-2516.

26. Deanfield, J.E., J.P. Halcox, and T.J. Rabelink, Endothelial function and dysfunction: Testing and clinical relevance. Circulation, 2007. 115(10): p. 1285-1295.

27. Adingupu, D.D., et al., Blood Oxygen Saturation After Ischemia is Altered With Abnormal Microvascular Reperfusion. Microcirculation, 2015. 22(4): p. 294-305.

28. Wright, C.I., C.I. Kroner, and R. Draijer, Non-invasive methods and stimuli for evaluating the skin's microcirculation. Journal of Pharmacological and Toxicological Methods, 2006. 54(1): p. 1-25.

29. Abraham, P., et al., Effect of skin temperature on skin endothelial function assessment. Microvascular Research, 2013. 88: p. 56-60.

30. Khan, F., et al., Impaired skin microvascular function in children, adolescents, and young adults with type I diabetes. Diabetes Care, 2000. 23(2): p. 215-220.

31. Schmiedel, O., M.L. Schroeter, and J.N. Harvey, Microalbuminuria in Type 2 diabetes indicates impaired microvascular vasomotion and perfusion. American Journal of Physiology - Heart and Circulatory Physiology, 2007. 293(6): p. H3424-H3431.

32. Minson, C.T., L.T. Berry, and M.J. Joyner, Nitric oxide and neurally mediated regulation of skin blood flow during local heating. Journal of Applied Physiology, 2001. 91(4): p. 1619-1626.

33. Cracowski, J.L., et al., Methodological issues in the assessment of skin microvascular endothelial function in humans. Trends in Pharmacological Sciences, 2006. 27(9): p. 503-508.

34. Tee, G.B.Y., et al., Dependence of human forearm skin postocclusive reactive hyperemia on occlusion time. Journal of Pharmacological and Toxicological Methods, 2004. 50(1): p. 73-78.

35. Morales, F., et al., How to assess post-occlusive reactive hyperaemia by means of laser Doppler perfusion monitoring: Application of a standardised protocol to patients with peripheral arterial obstructive disease. Microvascular Research, 2005. 69(1-2): p. 17-23.

36. Strain, W.D., et al., Albumin excretion rate and cardiovascular risk: Could the association be explained by early microvascular dysfunction? Diabetes, 2005. 54(6): p. 1816-1822.

37. Hecht, E., Electromagnetic theory, photons and light, in Optics. 2002, Addison Wesley.

38. Hecht, E., Laser and laserlight, in Optics. 2002, Addison Wesly.

39. Jacques, S.L., Optical properties of biological tissues: A review. Physics in Medicine and Biology, 2013. 58(11): p. R37-R61.

40. Cheong, W.F., S.A. Prahl, and A.J. Welch, A Review of the Optical-Properties of Biological Tissues. leee Journal of Quantum Electronics, 1990. 26(12): p. 2166-2185.

41. Doornbos, R.M.P., et al., The determination of in vivo human tissue optical properties and absolute chromophore concentrations using spatially resolved steady-state diffuse reflectance spectroscopy. Physics in Medicine and Biology, 1999. 44(4): p. 967-981.

42. Bargo, P.R., et al., In vivo determination of optical properties of normal and tumor tissue with white light reflectance and an empirical light transport model during endoscopy. Journal of biomedical optics, 2005. 10(3): p. 034018.

43. Finlay, J.C. and T.H. Foster, Hemoglobin oxygen saturations in phantoms and in vivo from measurements of steady-state diffuse reflectance at a single, short source-detector separation. Medical Physics, 2004. 31(7): p. 1949-1959.

44. Lister, T., P.A. Wright, and P.H. Chappell, Optical properties of human skin. Journal of Biomedical Optics, 2012. 17(9): p. 0909011-09090115. 
45. Anderson, R.R. and J.A. Parrish, The optics of human skin. Journal of Investigative Dermatology, 1981. 77(1): p. 13-19.

46. Prahl, S.A. Optical absorption of hemoglobin. 1999; Available from: http://omlc.org/spectra/hemoglobin/index.html.

47. Jacques, S. Melanosome absorption coefficient. 1998; Available from: http://omlc.org/spectra/melanin/mua.html.

48. Segelstein, D.J., The Complex Refractive Index of Water. 1981: Department of Physics. University of Missouri-Kansas City.

49. Van Veen, R.L.P., et al., Determination of visible near-IR absorption coefficients of mammalian fat using time- and spatially resolved diffuse reflectance and transmission spectroscopy. Journal of Biomedical Optics, 2005. 10(5).

50. Thody, A.J., et al., Pheomelanin as well as eumelanin is present in human epidermis. Journal of Investigative Dermatology, 1991. 97(2): p. 340-344.

51. Alaluf, S., et al., Ethnic variation in melanin content and composition in photoexposed and photoprotected human skin. Pigment Cell Research, 2002. 15(2): p. 112-118.

52. Ou-Yang, H., G. Stamatas, and N. Kollias, Spectral Responses of Melanin to Ultraviolet a Irradiation. Journal of Investigative Dermatology, 2004. 122(2): p. 492-496.

53. Jacques, S.L. Skin Optics. 1998; Available from: http://omlc.org/news/jan98/skinoptics.html.

54. Saager, R.B., et al., In vivo measurements of cutaneous melanin across spatial scales: Using multiphoton microscopy and spatial frequency domain spectroscopy. Journal of Biomedical Optics, 2015. 20(6).

55. Jacques, S.L. Extinction coefficient of melanin. 1998; Available from: http://omlc.org/spectra/melanin/extcoeff.html.

56. Zijlstra, W.G., A. Buursma, and O.W. van Assendelft, Visable and near infrared absorption spectra of human and animal haemoglobin. 2000: VSP.

57. Splinter, R. and B.A. Hooper, An Introduction to Biomedical Optics. 2006: Taylor \& Francis Group.

58. Saidi, I.S., S.L. Jacques, and F.K. Tittel, Mie and Rayleigh modeling of visible-light scattering in neonatal skin. Applied Optics, 1995. 34(31): p. 7410-7418.

59. Jacques, S. and S.A. Prahl. Some biological scatterers. 1998; Available from: http://omlc.org/education/ece532/class3/scatterers.html.

60. Hecht, E., The propagation of light, in Optics. 2002, Addison Wesly.

61. Henyey, L.G. and J.L. Greenstein, Diffuse radiation in the galaxy. Astrophysical Journal, 1941. 93: p. 70-83.

62. Reynolds, L.O. and N.J. McCormick, Approximate two-parameter phase function for light scattering. Journal of the Optical Society of America, 1980. 70(10): p. 1206-1212.

63. Larsson, M. and T. Strömberg, Toward a velocity-resolved microvascular blood flow measure by decomposition of the laser Doppler spectrum. Journal of biomedical optics, 2006. 11(1): p. 014024.

64. Forrester, A.T., Photoelectric mixing as a spectroscopic tool. JOSA, 1961. 51(3): p. 253-256.

65. Fredriksson, I., C. Fors, and J. Johansson, Laser Doppler flowmetry-a theoretical framework. 2007, Department of Biomedical Engineering, Linköping University.

66. Nilsson, G., Signal processor for laser Doppler tissue flowmeters. Medical and Biological Engineering and Computing, 1984. 22(4): p. 343-348.

67. Fredriksson, I., M. Larsson, and T. Strömberg, Laser Doppler Flowmetry, in Microcirculation imaging, M. Leahy, Editor. 2012, Wiley-Blackwell.

68. Mourant, J.R., et al., Predictions and measurements of scattering and absorption over broad wavelength ranges in tissue phantoms. Applied Optics, 1997. 36(4): p. 949-957.

69. Pogue, B.W. and M.S. Patterson, Review of tissue simulating phantoms for optical spectroscopy, imaging and dosimetry. Journal of Biomedical Optics, 2006. 11(4).

70. Yaroslavsky, I.V., et al., Inverse hybrid technique for determining the optical properties of turbid media from integrating-sphere measurements. Applied Optics, 1996. 35(34): p. 6797-6809. 
71. Wang, L. and S.L. Jacques, Error estimation of measuring total interaction coefficients of turbid media using collimated light transmission. Physics in medicine and biology, 1994. 39(12): p. 234954.

72. Kienle, A., et al., Spatially resolved absolute diffuse reflectance measurements for noninvasive determination of the optical scattering and absorption coefficients of biological tissue. Applied Optics, 1996. 35(13): p. 2304-2314.

73. Zaccanti, G., S. Del Bianco, and F. Martelli, Measurements of optical properties of high-density media. Applied optics, 2003. 42(19): p. 4023-30.

74. Lindbergh, T., et al., Spectral determination of a two-parametric phase function for polydispersive scattering liquids. Optics express, 2009. 17(3): p. 1610-21.

75. Driver, l., et al., The optical properties of aqueous suspensions of Intralipid, a fat emulsion. Physics in Medicine and Biology, 1989. 34(12): p. 1927-1930.

76. Jacques, S. Optical properties of Intralipid, an aqueous suspension of lipid droplets. 1998; Available from: http://omlc.org/spectra/intralipid/.

77. van Staveren, H.J., et al., Light scattering in Intralipid-10\% in the wavelength range of $400-1100 \mathrm{~nm}$. Appl Opt, 1991. 30(31): p. 4507-14.

78. Wang, L., S.L. Jacques, and L. Zheng, MCML-Monte Carlo modeling of light transport in multilayered tissues. Computer Methods and Programs in Biomedicine, 1995. 47(2): p. 131-146.

79. Prahl, S.A., et al., A Monte Carlo model of light propagation in tissue. Dosimetry of laser radiation in medicine and biology, 1989. 5: p. 102-111.

80. Alerstam, E., T. Svensson, and S. Andersson-Engels, Parallel computing with graphics processing units for high-speed Monte Carlo simulation of photon migration. Journal of Biomedical Optics, 2008. 13(6).

81. Alerstam, E., S. Andersson-Engels, and T. Svensson, White Monte Carlo for time-resolved photon migration. Journal of Biomedical Optics, 2008. 13(4).

82. Liu, Q. and N. Ramanujam, Scaling method for fast Monte Carlo simulation of diffuse reflectance spectra from multilayered turbid media. Journal of the Optical Society of America A: Optics and Image Science, and Vision, 2007. 24(4): p. 1011-1025.

83. Kienle, A. and M.S. Patterson, Determination of the optical properties of turbid media from a single Monte Carlo simulation. Physics in Medicine and Biology, 1996. 41(10): p. 2221-2227.

84. Zhu, C., et al., Diagnosis of breast cancer using diffuse reflectance spectroscopy: Comparison of a Monte Carlo versus partial least squares analysis based feature extraction technique. Lasers in surgery and medicine, 2006. 38(7): p. 714-24.

85. Qu, J., et al., Laser-induced fluorescence spectroscopy at endoscopy: tissue optics, Monte Carlo modeling, and in vivo measurements. Optical Engineering, 1995. 34(11): p. 3334-3343.

86. de Mul, F.F.M., et al., Laser Doppler velocimetry and Monte Carlo simulations on models for blood perfusion in tissue. Applied Optics, 1995. 34(28): p. 6595-6611.

87. Wojtkiewicz, S., et al., Evaluation of algorithms for microperfusion assessment by fast simulations of laser Doppler power spectral density. Physics in medicine and biology, 2011. 56(24): p. 7709.

88. Yao, G. and L.V. Wang, Monte Carlo simulation of an optical coherence tomography signal in homogeneous turbid media. Physics in Medicine and Biology, 1999. 44(9): p. 2307-2320.

89. Wang, Y., et al., Choosing optimal wavelength for photodynamic therapy of port wine stains by mathematic simulation. Journal of Biomedical Optics, 2011. 16(9).

90. Wang, L., S.L. Jacques, and L. Zheng, CONV - convolution for responses to a finite diameter photon beam incident on multi-layered tissues. Computer methods and programs in biomedicine, 1997. 54(3): p. 141-150.

91. Zhu, C. and Q. Liu, Review of Monte Carlo modeling of light transport in tissues. Journal of Biomedical Optics, 2013. 18(5): p. 050902-050902.

92. Rajaram, N., T.H. Nguyen, and J.W. Tunnell, Lookup table-based inverse model for determining optical properties of turbid media. Journal of Biomedical Optics, 2008. 13(5): p. 050501-050501-3. 
93. Saager, R.B., D.J. Cuccia, and A.J. Durkin, Determination of optical properties of turbid media spanning visible and near-infrared regimes via spatially modulated quantitative spectroscopy. J Biomed Opt, 2010. 15(1): p. 017012.

94. Graaff, R., et al., Reduced light-scattering properties for mixtures of spherical particles: a simple approximation derived from Mie calculations. Applied optics, 1992. 31(10): p. 1370-1376.

95. Mourant, J.R., et al., Mechanisms of light scattering from biological cells relevant to noninvasive optical-tissue diagnostics. Applied optics, 1998. 37(16): p. 3586-3593.

96. Lindbergh, T., et al., Improved model for myocardial diffuse reflectance spectra by including mitochondrial cytochrome aa3, methemoglobin, and inhomogenously distributed RBC. Journal of biophotonics, 2011. 4(4): p. 268-76.

97. Liu, H., et al., Influence of blood vessels on the measurement of hemoglobin oxygenation as determined by time-resolved reflectance spectroscopy. Medical physics, 1995. 22(8): p. 1209-17.

98. Fredriksson, I., M. Larsson, and T. Strömberg. Accuracy of vessel diameter estimated from a vessel packaging compensation in diffuse reflectance spectroscopy. in European Conference on Biomedical Optics. 2011. Optical Society of America.

99. Fredriksson, I., et al., Inverse Monte Carlo in a multilayered tissue model: merging diffuse reflectance spectroscopy and laser Doppler flowmetry. J Biomed Opt, 2013. 18(12): p. 127004.

100. Rajaram, N., et al., Experimental validation of the effects of microvasculature pigment packaging on in vivo diffuse reflectance spectroscopy. Lasers in surgery and medicine, 2010. 42(7): p. 680-8.

101. Van Veen, R.L.P., W. Verkruysse, and H.J.C.M. Sterenborg, Diffuse-reflectance spectroscopy from 500 to $1060 \mathrm{~nm}$ by correction for inhomogeneously distributed absorbers. Optics Letters, 2002. 27(4): p. 246-248.

102. Talsma, A., B. Chance, and R. Graaff, Corrections for inhomogeneities in biological tissue caused by blood vessels. Journal of the Optical Society of America. A, Optics, image science, and vision, 2001. 18(4): p. 932-9.

103. Svaasand, L.O., et al., Therapeutic response during pulsed laser treatment of port-wine stains: Dependence on vessel diameter and depth in dermis. Lasers in Medical Science, 1995. 10(4): p. 235243.

104. Fredriksson, I., M. Larsson, and T. Strömberg, Inverse Monte Carlo method in a multilayered tissue model for diffuse reflectance spectroscopy. Journal of Biomedical Optics, 2012. 17(4).

105. Fredriksson, l., et al. Improved calibration procedure for laser Doppler perfusion monitors. in Progress in Biomedical Optics and Imaging - Proceedings of SPIE. 2011.

106. Kleinbaum, D.G., L.L. Kupper, and K.E. Muller, Applied regression analysis and other multivariable methods, ed. D.G. Kleinbaum, L.L. Kupper, and K.E. Muller. 1988: PWS Publishing Co. 718.

107. Zonios, G. and A. Dimou, Modeling diffuse reflectance from homogeneous semi-infinite turbid media for biological tissue applications: a Monte Carlo study. Biomedical Optics Express, 2011. 2(12): p. 3284-3294.

108. Hennessy, R., M.K. Markey, and J.W. Tunnell, Impact of one-layer assumption on diffuse reflectance spectroscopy of skin. Journal of Biomedical Optics, 2015. 20(2): p. 027001-027001.

109. Liu, Q. and N. Ramanujam, Sequential estimation of optical properties of a two-layered epithelial tissue model from depth-resolved ultraviolet-visible diffuse reflectance spectra. Applied Optics, 2006. 45(19): p. 4776-4790.

110. Pham, T.H., et al., Quantifying the properties of two-layer turbid media with frequency-domain diffuse reflectance. Applied Optics, 2000. 39(25): p. 4733-4745.

111. Sharma, M., et al., Verification of a two-layer inverse Monte Carlo absorption model using multiple source-detector separation diffuse reflectance spectroscopy. Biomedical Optics Express, 2014. 5(1): p. 40-53.

112. Wang, Q., K. Shastri, and T.J. Pfefer, Experimental and theoretical evaluation of a fiber-optic approach for optical property measurement in layered epithelial tissue. Applied Optics, 2010. 49(28): p. 5309-5320. 
113. Yudovsky, D. and A.J. Durkin, Spatial frequency domain spectroscopy of two layer media. Journal of Biomedical Optics, 2011. 16(10).

114. Tseng, S.H., et al., Chromophore concentrations, absorption and scattering properties of human skin in-vivo. Optics Express, 2009. 17(17): p. 14599-14617.

115. Bashkatov, A.N., et al., Optical properties of human skin, subcutaneous and mucous tissues in the wavelength range from 400 to $2000 \mathrm{~nm}$. Journal of Physics D: Applied Physics, 2005. 38(15): p. 2543-2555.

116. Häggblad, E., et al., Myocardial tissue oxygenation estimated with calibrated diffuse reflectance spectroscopy during coronary artery bypass grafting. Journal of biomedical optics, 2008. 13(5): p. 054030.

117. Beckert, S., et al., The impact of the micro-lightguide $\mathrm{O} 2 \mathrm{C}$ for the quantification of tissue ischemia in diabetic foot ulcers. Diabetes Care, 2004. 27(12): p. 2863-2867.

118. Bernjak, A., et al., Coherence between fluctuations in blood flow and oxygen saturation. Fluctuation and Noise Letters, 2012. 11(1).

119. Yvonne-Tee, G.B., et al., Reproducibility of different laser Doppler fluximetry parameters of postocclusive reactive hyperemia in human forearm skin. Journal of Pharmacological and Toxicological Methods, 2005. 52(2): p. 286-292.

120. Wright, L.P., et al. Comparison of TCPO2 and StO2 using the blood oxygen dissociation curve. 2006.

121. Saager, R.B., et al., Method for depth-resolved quantitation of optical properties in layered media using spatially modulated quantitative spectroscopy. J Biomed Opt, 2011. 16(7): p. 077002.

122. Roustit, M., et al., Reproducibility and methodological issues of skin post-occlusive and thermal hyperemia assessed by single-point laser Doppler flowmetry. Microvascular Research, 2010. 79(2): p. 102-108.

123. Sunar, U., et al., Noninvasive diffuse optical measurement of blood flow and blood oxygenation for monitoring radiation therapy in patients with head and neck tumors: A pilot study. Journal of Biomedical Optics, 2006. 11(6).

124. Verdecchia, K., et al., Quantifying the cerebral metabolic rate of oxygen by combining diffuse correlation spectroscopy and time-resolved near-infrared spectroscopy. Journal of Biomedical Optics, 2013. 18(2).

125. Zhou, C., et al., Diffuse optical monitoring of blood flow and oxygenation in human breast cancer during early stages of neoadjuvant chemotherapy. Journal of Biomedical Optics, 2007. 12(5).

126. Fredriksson, l., et al., Reduced arteriovenous shunting capacity after local heating and redistribution of baseline skin blood flow in type 2 diabetes assessed with velocity-resolved quantitative laser Doppler flowmetry. Diabetes, 2010. 59(7): p. 1578-84.

127. Brunt, V.E. and C.T. Minson, KCa channels and epoxyeicosatrienoic acids: Major contributors to thermal hyperaemia in human skin. Journal of Physiology, 2012. 590(15): p. 3523-3534.

128. Choi, P.J., et al., New approach to measure cutaneous microvascular function: An improved test of NO-mediated vasodilation by thermal hyperemia. Journal of Applied Physiology, 2014. 117(3): $p$. 277-283.

129. Kellogg Jr, D.L., et al., Role of nitric oxide in the vascular effects of local warming of the skin in humans. Journal of Applied Physiology, 1999. 86(4): p. 1185-1190.

130. Carter, S.J. and G.J. Hodges, Sensory and sympathetic nerve contributions to the cutaneous vasodilator response from a noxious heat stimulus. Experimental Physiology, 2011. 96(11): p. 12081217.

131. Wojtkiewicz, S., et al., Assessment of speed distribution of red blood cells in the microvascular network in healthy volunteers and type 1 diabetes using laser Doppler spectra decomposition. Physiological Measurement, 2014. 35(2): p. 283-295. 


\section{Papers}

The articles associated with this thesis have been removed for copyright reasons. For more details about these see:

http://urn.kb.se/resolve?urn=urn:nbn:se:liu:diva-127691 